\title{
ROBINSON-SCHENSTED CORRESPONDENCE FOR UNIT INTERVAL ORDERS
}

\author{
DONGKWAN KIM AND PAVLO PYLYAVSKYY
}

\begin{abstract}
The Stanley-Stembridge conjecture associates a symmetric function to each natural unit interval order $\mathcal{P}$. In this paper, we define relations à la Knuth on the symmetric group for each $\mathcal{P}$ and conjecture that the associated $\mathcal{P}$-Knuth equivalence classes are Schur-positive, refining theorems of Gasharov, Brosnan-Chow, Guay-Paquet, and Shareshian-Wachs. The resulting equivalence graphs fit into the framework of D graphs studied by Assaf. Furthermore, we conjecture that the Schur expansion is given by column-readings of $\mathcal{P}$-tableaux that occur in the equivalence class. We prove these conjectures for $\mathcal{P}$ avoiding two specific suborders by introducing $\mathcal{P}$-analog of Robinson-Schensted insertion, giving an answer to a long standing question of Chow.
\end{abstract}

1. Introduction

2. Definitions and notations

3. Natural unit interval order

4. $\mathcal{P}$-Knuth equivalence and the main theorem 9

5. Column insertion algorithm 14

6. $\mathcal{P}$-Robinson-Schensted algorithm 19

7. Examples 20

8. Proof of Proposition 4.10, 5.5, and 5.6 29

9. Proof of Theorem 6.1 and 4.13

References

\section{INTRODUCTION}

Since its formulation in 1993, Stanley-Stembridge conjecture [SS93, Conjecture 5.5] has been one of the most intriguing problems in algebraic combinatorics. Interest in it was greatly strengthened when Shareshian and Wachs [SW16] related the conjecture to Hessenberg varieties. The original conjecture was shown by Guay-Paquet GP13] to be equivalent to saying that chromatic symmetric functions of incomparability graphs of unit interval orders are positive combinations of elementary symmetric functions. Shareshian and Wachs realized that essentially the same symmetric functions arise as Frobenius characters of actions of symmetric groups on cohomology rings of Hessenberg varieties, as studied by Tymoczko Tym08. Shareshian-Wachs conjecture was proved by Brosnan and Chow [BC18, and independently by Guay-Paquet [GP16]. On the combinatorial level the results of Brosnan-Chow and Guay-Paquet imply a graded refinement of the Schur positivity result

Date: May 15, 2020. 
of Gasharov Gas96. It also provides useful tools to understand combinatorics in terms of geometry, i.e. theory of perverse sheaves and geometric properties of (regular) Hessenberg varieties.

The original Stanley-Stembridge conjecture, nowadays usually stated in terms of positivity in complete homogenous symmetric functions, remains open except for special cases, see GebhardSagan [GS01, Dahlberg-van Willigenburg [DvW18], Harada-Precup [HP19], Cho-Huh [CH19b], Cho-Hong [CH19a, etc.

In an independent development, Assaf [Ass15, Ass17, has introduced a beautiful theory of D graphs to address Schur positivity questions in symmetric functions, such as Macdonald polynomials, LLT polynomials, and $k$-Schur functions. While as shown by Blasiak Bla16 getting exactly the right axiomatization to address those questions can be very challenging, Assaf's work provides a very useful framework. In particular her characterization of dual equivalence graphs has been used in a variety of contexts, see for example Chmutov [Chm15] and Roberts Rob14. Assaf's ideas were further developed by Blasiak-Fomin [BF17] and others.

In this paper we combine the two lines of research. Specifically, for each unit interval order $\mathcal{P}$ we define an analog of Knuth moves. The resulting $\mathcal{P}$-Knuth equivalence classes of permutations satisfy correct axioms to fit into the framework of D graphs. We conjecture that via the standard map from permutations to quasisymmetric functions the images of $\mathcal{P}$-Knuth equivalence classes are symmetric and Schur positive. This is a refinement of results of Gasharov, Brosnan-Chow, and Guay-Paquet. Furthermore, we conjecture that the decomposition into Schur functions can be read off from column reading words of $\mathcal{P}$-tableaux that occur in the equivalence class.

We prove this Schur positivity conjecture for a special class of unit interval orders $\mathcal{P}$. For that purpose we introduce an analog of Robinson-Schensted insertion that preserves descents, solving an open problem dating back to the works of Sundquist-Wagner-West and Chow. The 1997 work of Sundquist-Wagner-West [SWW97] constructs a version of Robinson-Schensted insertion for unit interval orders, however in general their algorithm does not preserve descents, and thus cannot be used to derive Schur positivity results. Chow Cho99 proved that Sundquist-Wagner-West does preserve descents under a very restrictive condition - in our terminology his condition is to avoid a suborder isomorphic to $\mathcal{P}_{(2,1), 4}$. Chow implicitly states in his paper the question of constructing Robinson-Schensted correspondence that preserves descents when $\mathcal{P}$ avoids a less restrictive pattern $\mathcal{P}_{(3,1,1), 5}$. In this paper we solve this problem for unit interval orders that avoid both $\mathcal{P}_{(3,1,1), 5}$ and $\mathcal{P}_{(4,2,1,1), 6}$. As a result, in those cases we are able to prove Schur positivity of the $\mathcal{P}$-Knuth equivalence classes.

This project started as an attempt to prove Stanley-Stembridge conjecture. This goal remains elusive, as it would require introducing an affine analog of $\mathcal{P}$-Knuth equivalence classes and proving their $h$-positivity. We expect this to be strictly harder than proving Schur positivity of the $\mathcal{P}$ Knuth equivalence graphs introduced in this paper, and even that remains open in full generality. Nevertheless, $\mathcal{P}$-Knuth equivalence classes seem to be interesting objects of their own, perhaps having geometric meaning in terms of (equivariant) cohomology and moment graphs of Hessenberg varieties. We hope that understanding $\mathcal{P}$-Knuth equivalence classes, and in particular proving Conjecture 4.14, will shed new light on Stanley-Stembridge conjecture.

The paper proceeds as follows. In Section 2 we recall some standard combinatorial notions such as partitions, partial orders and tableaux. In Section 3 we recall properties and characterizations of natural unit interval orders. We also introduce an important class of natural unit interval orders called ladders, as well as ladder-climbing property. In Section 4 we introduce $\mathcal{P}$-Knuth equivalence 
classes and state the main theorem 4.13 In Section 5 we introduce column insertion procedure, which is then used in Section 6 to define the full $\mathcal{P}$-Robinson-Schensted insertion algorithm. Section 7 is filled with examples illustrating everything introduced in the previous sections. In Sections 8 and 9 we give proofs of the results from previous sections.

\section{Definitions and notations}

For $a, b \in \mathbb{Z}$, we set $[a, b]:=\{x \in \mathbb{Z} \mid a \leq x \leq b\}$. For a set $X$, we let $|X|$ be its cardinal.

2.1. Partitions. A partition is a finite sequence of integers $\lambda=\left(\lambda_{1}, \lambda_{2}, \ldots, \lambda_{a}\right)$ such that $\lambda_{1} \geq$ $\lambda_{2} \geq \cdots \geq \lambda_{a}>0$. In such a case, we set its length to be $a$ (denoted $l(\lambda)$ ) and its size to be $\lambda_{1}+\lambda_{2}+\cdots+\lambda_{a}$ (denoted $|\lambda|$ ). When $|\lambda|=n$, we also write $\lambda \vdash n$. If $i>l(\lambda)$, we set $\lambda_{i}=0$. We write $\lambda^{\prime}$ to denote the conjugate partition of $\lambda$. We define the staircase partition $\operatorname{Stair}(n)$ to be $(n-1, n-2, \ldots, 2,1)$. For two partitions $\lambda$ and $\mu$, we write $\lambda \subset \mu$ if $\lambda_{i} \leq \mu_{i}$ for all $i \in \mathbb{Z}_{>0}$. Pictorially, it means that the Young diagram of $\mu$ contains that of $\lambda$.

2.2. Partial orders. We use the symbols $\geq,>, \leq$, and $<$ for the usual order on $\mathbb{R}$. However, throughout this paper we discuss various partial orders, for which new symbols are necessary in order to avoid conflict. Namely, suppose that a partial order $\mathcal{P}$ on $[1, n]$ is given. For $a, b \in[1, n]$, we write

(1) $a \prec_{\mathcal{P}} b$ (or $b \succ_{\mathcal{P}} a$ ) if $a$ is smaller than $b$ with respect to $\mathcal{P}$,

(2) $a \leftarrow_{\mathcal{P}} b$ (or $b \rightarrow_{\mathcal{P}} a$ ) if $a<b$ and $a \prec_{\mathcal{P}} b$,

(3) $a \leftrightarrow-\mathcal{P} b$ (or $b-\rightarrow_{\mathcal{P}} a$ ) if $a<b$ but $a \nprec_{\mathcal{P}} b$, and

(4) $a--_{\mathcal{P}} b$ if $a$ and $b$ are not comparable with respect to $\mathcal{P}$ (and $a \neq b$ ).

If there is no confusion we drop the subscript $\mathcal{P}$ from each symbol.

For a partial order $\mathcal{P}$ on a set $X$ and its subset $Y \subset X$, the restriction of $\mathcal{P}$ to $Y$, denoted $\left.\mathcal{P}\right|_{Y}$, is well-defined. For two partial orders $\mathcal{P}$ on $[1, n]$ and $\mathcal{P}^{\prime}$ on $[1, m]$, we say that $\mathcal{P}$ avoids $\mathcal{P}^{\prime}$ or $\mathcal{P}$ is $\mathcal{P}^{\prime}$-avoiding if restriction of $\mathcal{P}$ to any subset of $[1, n]$ (of cardinal $m$ ) is not isomorphic to $\mathcal{P}^{\prime}$.

2.3. Symmetric groups and words. In this paper, a word means a finite sequence. For a word $\alpha=\left(\alpha_{1}, \alpha_{2}, \ldots, \alpha_{k}\right)$, we also write $\alpha=\alpha_{1} \alpha_{2} \cdots \alpha_{k}$ to simplify notations. For a word $\alpha$, we denote by $\underline{\alpha}$ the corresponding underlying set. We let $|\alpha| \in \mathbb{N}$ be the length of $\alpha$. If $|\alpha|=0$, then we also write $\alpha=\emptyset$. By a subword of $\alpha$, we mean a word $\left(\alpha_{i_{1}}, \alpha_{i_{2}}, \ldots, \alpha_{i_{s}}\right)$ such that $1 \leq i_{1}<i_{2}<\cdots<i_{s} \leq k$. For two words $\alpha$ and $\beta$, we define $\alpha+\beta$ to be their concatenation.

Let $\mathfrak{S}_{n}$ be the symmetric group permuting $[1, n]$. We identify elements in $\mathfrak{S}_{n}$ with the words in which each of $1,2, \ldots, n$ appears once. For $w=w_{1} w_{2} \cdots w_{n} \in \mathfrak{S}_{n}$ and a partial order $\mathcal{P}$ defined on $[1, n]$, we set

- its $\mathcal{P}$-descent to be $\operatorname{des}_{\mathcal{P}}(w)=\left\{i \in[1, n-1] \mid w_{i} \succ_{\mathcal{P}} w_{i+1}\right\}$,

- its genuine $\mathcal{P}$-inversion to be

$$
\begin{aligned}
\operatorname{g-inv}_{\mathcal{P}}(w)= & \left\{(i, j) \in[1, n]^{2} \mid i \rightarrow_{\mathcal{P}} j, i \text { appears before } j \text { in } w,\right. \\
& \text { and there do not exist any subword } i a_{1} a_{2} \cdots a_{k} j \text { of } w \text { such that } \\
& \left.i--_{\mathcal{P}} a_{1-\mathcal{P}_{\mathcal{P}}} a_{2}--_{\mathcal{P}} \cdots-\mathcal{P}_{\mathcal{P}} a_{k-\mathcal{P}_{\mathcal{P}}} j\right\},
\end{aligned}
$$


- its (genuine) $\mathcal{P}$-height to be (if $\mathrm{g}$-inv ${ }_{\mathcal{P}}(w)=\emptyset$ then $\mathrm{g}^{-h t_{\mathcal{P}}}(w)=1$ and otherwise)

$$
\begin{array}{r}
\mathrm{g}-\mathrm{ht}_{\mathcal{P}}(w)=\max \left\{k \in \mathbb{N} \mid \text { there exist } a_{1}, a_{2}, \ldots, a_{k-1}, a_{k}\right. \text { such that } \\
\left(a_{1}, a_{2}\right), \ldots,\left(a_{k-1}, a_{k}\right) \in \mathrm{g}_{\left.-\operatorname{inv}_{\mathcal{P}}(w)\right\},}
\end{array}
$$

- its fake $\mathcal{P}$-inversion to be $\operatorname{finv}_{\mathcal{P}}(w)=\left\{(i, j) \in[1, n]^{2} \mid i-\rightarrow \mathcal{P} j, w^{-1}(i)<w^{-1}(j)\right\}$, and

- its (fake) $\mathcal{P}$-inversion number to be $\left|f_{-}-\operatorname{inv}_{\mathcal{P}}(w)\right|$.

For example, if $\mathcal{P}$ is the usual order on $[1, n]$ then $\operatorname{g-inv}_{\mathcal{P}}(w)$ is the set of usual inversions in $w$, g-ht $(w)$ is the length of the longest decreasing subword of $w$, and $\operatorname{f}_{-} \operatorname{inv}_{\mathcal{P}}(w)=\emptyset$. On the other hand, if $\mathcal{P}$ is the trivial order on $[1, n]$ then $g-\operatorname{inv}_{\mathcal{P}}(w)=\emptyset, \mathrm{g}-\mathrm{ht}_{\mathcal{P}}(w)=1$, and $\mathrm{f}-\operatorname{inv}_{\mathcal{P}}(w)$ is the set of usual inversions in $w$. In addition, if $\mathcal{P}$ is defined with respect to the Hasse diagram in Figure 1 and $w=(9,5,1,8,4,7,3,6,2)$ then

- $\operatorname{des} \mathcal{P}(w)=\{1,2,4,6,8\}$,

- $\operatorname{g-inv}_{\mathcal{P}}(w)=\{(9,5),(9,4),(9,3),(9,2),(9,1),(8,4),(8,3),(8,2),(7,3),(7,2),(6,2),(5,1)\}$,

- g-ht $_{\mathcal{P}}(w)=3(=$ the length of $(9,5,1))$,

- $\mathrm{f}_{-} \operatorname{inv}_{\mathcal{P}}(w)=\{(3,2),(4,3),(5,3),(5,4),(7,6),(8,6),(8,7),(9,8)\}$, and

- $\left|\mathrm{f}-\operatorname{inv}_{\mathcal{p}}(w)\right|=8$.

Remark. We adopt the convention that if $i \rightarrow j$ in the Hasse diagram of an order $\mathcal{P}$ then $i$ is greater than $j$ with respect to $\mathcal{P}$, which is in accordance with the notation $i \rightarrow_{\mathcal{P}} j$.

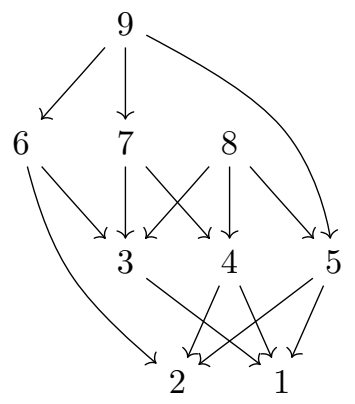

FiguRE 1

2.4. Standard and $\mathcal{P}$-tableaux. A tableau $T$ is said to satisfy the $\mathcal{P}$-tableau condition if for two entries $i, j \in[1, n]$ adjacent in $T$, (1) if $i$ is above $j$ then $i \prec_{\mathcal{P}} j$, and (2) if $i$ is left to $j$ then $i \nsucc_{\mathcal{P}} j$. That is, entries in $T$ are increasing along columns and nondecreasing along rows with respect to $\mathcal{P}$. Such a tableau $T$ is called a $\mathcal{P}$-tableau if in addition it contains each entry of $[1, n]$ exactly once. (Note that our definition is a conjugated version of the one in Gas96, Theorem 3].) We denote by $\mathcal{P}-\mathrm{Tab}_{n}$ (resp. $\mathcal{P}-\mathrm{Tab}_{\lambda}$ ) the set of $\mathcal{P}$-tableaux of size $n$ (resp. of shape $\lambda$ ). Similarly, we denote by $\mathrm{SYT}_{n}$ (resp. $\mathrm{SYT}_{\lambda}$ ) the set of standard Young tableaux of size $n$ (resp. of shape $\lambda$ ). Note that if $\mathcal{P}$ is the usual order on $[1, n]$ then $\mathcal{P}$-tableaux are exactly standard Young tableaux.

For a tableau $T$, we often identify each of its columns with its reading word from bottom to top and also identify $T$ with the sequence of its columns. In addition, we define the reading word of $T$, denoted $\operatorname{read}(T)$, to be the concatenation of column reading words from bottom to top. We define the descent of $T \in \mathrm{SYT}_{n}$ to be $\operatorname{des}(T)=\{i \in[1, n-1] \mid i$ is in a higher row than that of $i+1\}$. 
For example, if $T=$\begin{tabular}{l|l|l|l|l}
1 & 3 & 5 & 8
\end{tabular} then $T$ is identified with $((4,2,1),(6,3),(7,5),(8))$, the reading word of $T$ is $\operatorname{read}(T)=(4,2,1,6,3,7,5,8)$, and $\operatorname{des}(T)=\{1,3,5\}$.

For a partial order $\mathcal{P}$ on $[1, n]$ and $T \in \mathcal{P}$-Tab ${ }_{n}$, we define its fake $\mathcal{P}$-inversion to be $\operatorname{finv}_{\mathcal{P}}(T)=$ $\left\{(i, j) \in[1, n]^{2} \mid i \rightarrow \mathcal{P} j\right.$, the column of $i$ is left to that of $\left.j\right\}$ and its fake $\mathcal{P}$-inversion number to be $\left|f_{\text {-inv }}(T)\right|$. Since each column of a $\mathcal{P}$-tableau is a chain in $\mathcal{P}$, it is easy to show that $\operatorname{f}_{\mathcal{P}} \operatorname{inv}_{\mathcal{P}}(T)=$ $\mathrm{f}_{\text {-inv }}(\operatorname{read}(T))$ for any $\mathcal{P}$-tableau $T$. For example, if $\mathcal{P}$ is given again by Figure 1 and $T=$\begin{tabular}{|l|l|l|l|}
\hline & 4 & 3 & 2 \\
\hline 5 & 8 & 7 & 6 \\
\hline
\end{tabular}

then $\mathrm{f}_{-\operatorname{inv}_{\mathcal{P}}}(T)=\mathrm{f}_{-\operatorname{inv}_{\mathcal{P}}}(\operatorname{read}(T))=\{(3,2),(4,3),(5,3),(5,4),(7,6),(8,6),(8,7),(9,8)\}$, and thus $\left|\mathrm{f}_{-\operatorname{inv}_{\mathcal{P}}}(T)\right|=8$.

2.5. Schur and fundamental quasi-symmetric functions. For a partition $\lambda$, we set $s_{\lambda}$ to be the Schur function corresponding to $\lambda$. For a composition $\mu$, we set $F_{\mu}$ to be the fundamental quasi-symmetric function corresponding to $\mu$ defined by Gessel.

\section{NATURAL Unit INTERVAl ORDER}

In this section we recall the notion of natural unit interval orders and some of its properties. Also, we introduce a ladder order which plays a prominent role in this paper.

3.1. Three equivalent definitions of natural unit interval orders. Here we define natural unit interval orders in three different ways. We refer readers to [SW16, Section 4] for the proof that these definitions are indeed equivalent.

\subsubsection{Definition in terms of unit intervals.}

Definition 3.1. We say that $\mathcal{P}$ on $[1, n]$ is a natural unit interval order if it there exist $n$ real numbers $y_{1}<y_{2}<\cdots<y_{n}$ such that $i \prec \mathcal{P} j \Leftrightarrow y_{i}+1<y_{j}$.

Pictorially, one may regard $y_{1}, \ldots, y_{n}$ as the starting points of unit intervals $I_{1}=\left[y_{1}, y_{1}+1\right], I_{2}=$ $\left[y_{2}, y_{2}+1\right], \ldots, I_{n}=\left[y_{n}, y_{n}+1\right]$. Then the above definition translates to the following.

(1) If $i<j$, then $I_{i}$ should start before $I_{j}$ in the real line.

(2) We have $i \prec_{\mathcal{P}} j$ if and only if $I_{j}$ starts after $I_{i}$ ends in the real line.

For example, Figure 2 shows an arrangement of unit intervals and the corresponding natural unit interval order.

3.1.2. Definition using partitions. For $n \in \mathbb{Z}_{>0}$, let $\lambda$ be a partition contained in $\operatorname{Stair}(n)$. We define the partial order $\mathcal{P}_{\lambda, n}$ on $[1, n]$ such that $a \prec_{\mathcal{P}} b$ if and only if $a \leq \lambda_{n+1-b}$. In other words, we have $\left[1, \lambda_{i}\right]=\left\{x \in[1, n] \mid x \prec_{\mathcal{P}} n+1-i\right\}$. For example, when $n=\overline{5}$ and $\lambda=(3,1)$ then $\mathcal{P}_{\lambda, n}$ is given as in Figure 3

Definition 3.2. We say that a partial order $\mathcal{P}$ on $[1, n]$ is a natural unit interval order if $\mathcal{P}=\mathcal{P}_{\lambda, n}$ for some $\lambda \subset \operatorname{Stair}(n)$. 


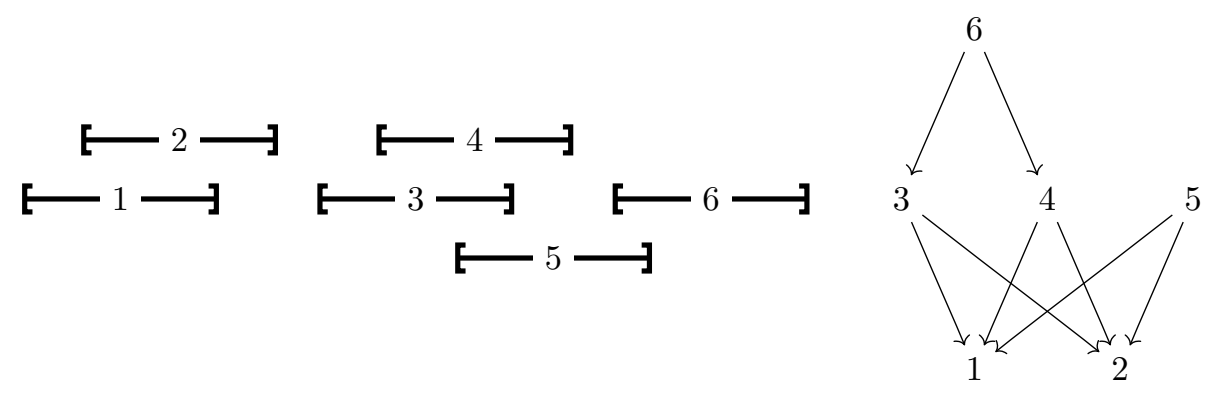

FIgURE 2. Natural unit interval order in terms of unit intervals
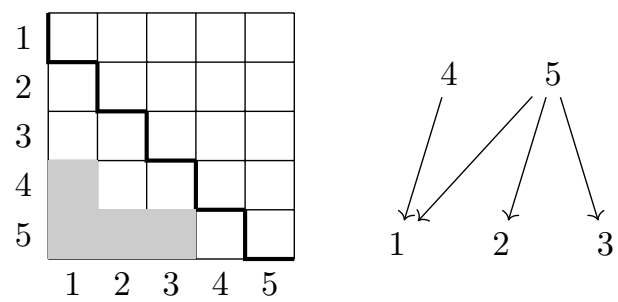

FiguRE 3. Natural unit interval order in terms of partitions

3.1.3. Defining properties. One may also define natural unit interval orders by imposing certain conditions on a partial order, namely:

Definition 3.3. We say that a partial order $\mathcal{P}$ on $[1, n]$ is a natural unit interval order if

- the usual order is a linearization of $\mathcal{P}$, i.e. if $a \prec_{\mathcal{P}} b$ then $a<b$, and

- if $b \leftarrow \mathcal{P} c, a--_{\mathcal{P}} b$, and $a--_{\mathcal{P}} c$, then $b<a<c$.

Because of the first condition, $\leftarrow_{\mathcal{P}}, \rightarrow_{\mathcal{P}}$ are equivalent to $\prec_{\mathcal{P}}, \succ_{\mathcal{P}}$ when we consider natural unit interval orders. From now on we usually use the former rather than the latter for such orders. Also, hereafter we refer to the second condition as Condition $(\pitchfork)$. For example, if $\mathcal{P}$ is a natural unit interval order and $a, b, c \in[1, n]$ satisfy $b \leftarrow \mathcal{P} c$ and $a<b$, then $a \leftarrow \mathcal{P} c$ by Condition ( $\pitchfork$ ).

3.2. $(3+1)$ and $(2+2)$ avoidance. There is another characterization of natural unit interval orders in terms of suborder avoidance. Indeed, it is essentially proved in SS58 that a partial order $\mathcal{P}$ on $[1, n]$ is a natural unit interval order if and only if the usual order is a linearization of $\mathcal{P}$ and $\mathcal{P}$ avoids suborders " $(3+1)$ " (disjoint union of a chain of length 3 and an element) and " $(2+2)$ " (disjoint union of two chains of length 2). Here we prove only one direction which will be useful later on.

Lemma 3.4 (See Figure 4). Suppose that $\mathcal{P}$ is a natural unit interval order on $[1, n]$. Then,

(1) there does not exist $a, b, c, d \in[1, n]$ such that $a \rightarrow_{\mathcal{P}} b \rightarrow_{\mathcal{P}} c, a--_{\mathcal{P}} d$, and $d--_{\mathcal{P}} c$, and

(2) there does not exist $a, b, c, d \in[1, n]$ such that $a \rightarrow_{\mathcal{P}} b, c \rightarrow_{\mathcal{P}} d, c--_{\mathcal{P}} b$, and $a--_{\mathcal{P}} d$. 
Proof. (1) If $b>d$ (resp. $b=d$ ) then $a \rightarrow_{\mathcal{P}} d$ by Condition (†) applied to $\{a, b, d\}$ (resp. since $a \rightarrow_{\mathcal{P}} b$ ) which is absurd. Similarly, if $b<d$ then $d \rightarrow_{\mathcal{P}} c$ by Condition ( $\pitchfork$ ) which is again impossible. (2) If $c>a$ (resp. $c=a$ ) then $c \rightarrow_{\mathcal{P}} b$ by Condition ( $\pitchfork$ ) applied to $\{a, b, c\}$ (resp. since $a \rightarrow_{\mathcal{P}} b$ ) which is absurd, and thus $c<a$. However, by symmetry we should have $a<c$ as well, which is again impossible.
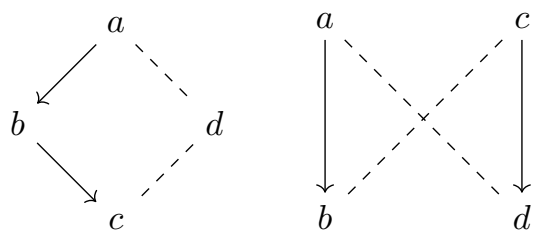

FiguRE 4. $(3+1)$ - and (2+2)-posets

3.3. Properties of $\mathcal{P}$-tableaux. We discuss some properties of $\mathcal{P}$-tableaux for natural unit interval orders. These will be frequently used in the later part of this paper without reminder.

Lemma 3.5. Suppose that $\left(a_{p}, \ldots, a_{1}\right)$ and $\left(b_{q}, \ldots b_{1}\right)$ are two adjacent columns in a $\mathcal{P}$-tableau such that the former is on the left of the latter.

(1) If $a_{i}>b_{j}$ then $i \geq j$

(2) If $a_{i} \rightarrow_{\mathcal{P}} b_{j}$ then $i>j$.

(3) If $i<j$ then $a_{i}<b_{j}$.

Proof. For (1), suppose $a_{i}>b_{j}$ and $i<j$. Then $b_{i} \leftarrow_{\mathcal{P}} b_{j}$ thus $a_{i} \rightarrow_{\mathcal{P}} b_{i}$ by Condition ( $\left.\pitchfork\right)$, which contradicts the $\mathcal{P}$-tableau condition. This proves (1). For (2), suppose $a_{i} \rightarrow_{\mathcal{P}} b_{j}$ and $i \leq j$. Then $b_{i} \leq b_{j}$ thus $a_{i} \rightarrow_{\mathcal{P}} b_{i}$ by Condition $(\pitchfork)$, which contradicts the $\mathcal{P}$-tableau condition. This proves (2). For (3), suppose $i<j$ and $a_{i} \geq b_{j}$. Then $b_{i} \leftarrow \mathcal{P} b_{j}$ thus $a_{i} \rightarrow_{\mathcal{P}} b_{i}$ by Condition ( $\pitchfork$ ), which contradicts the $\mathcal{P}$-tableau condition. This proves (3).

3.4. Ladders. We define a special kind of a natural unit interval order called a ladder order.

Definition 3.6. A partial order $\mathcal{P}$ is called a ladder order if it is isomorphic to $\mathcal{P}_{\operatorname{Stair}(m-1), m}$ for some $m \in \mathbb{N}$.

Figure 5 shows the partial order $\mathcal{P}_{\text {Stair(9),10 }}$ which is by definition a ladder order, and the partition and the unit interval arrangement defining it. The term "ladder" is inspired from the shapes of its Hasse diagram and the corresponding unit interval arrangement.

Definition 3.7. For a partial order $\mathcal{P}$ defined on $X$, we say that $A$ is a ladder in $\mathcal{P}$ if $\left.\mathcal{P}\right|_{A}$ is a ladder order.

For example, if $\mathcal{P}=\mathcal{P}_{\text {Stair }(m-1), m}$ then ladders in $\mathcal{P}$ are exactly $[a, b]$ for some $a, b \in[1, m]$. Let us describe some basic properties of ladders. Later we will frequently use these properties without reminder.

Lemma 3.8. Suppose that $\mathcal{P}$ is a natural unit interval order on $[1, n]$ and assume that $y_{1}, \ldots, y_{k} \in$ $[1, n]$ such that $y_{1}<y_{2}<\cdots<y_{k}$ and $\left\{y_{1}, \ldots, y_{k}\right\}$ is a ladder in $\mathcal{P}$. Then, 


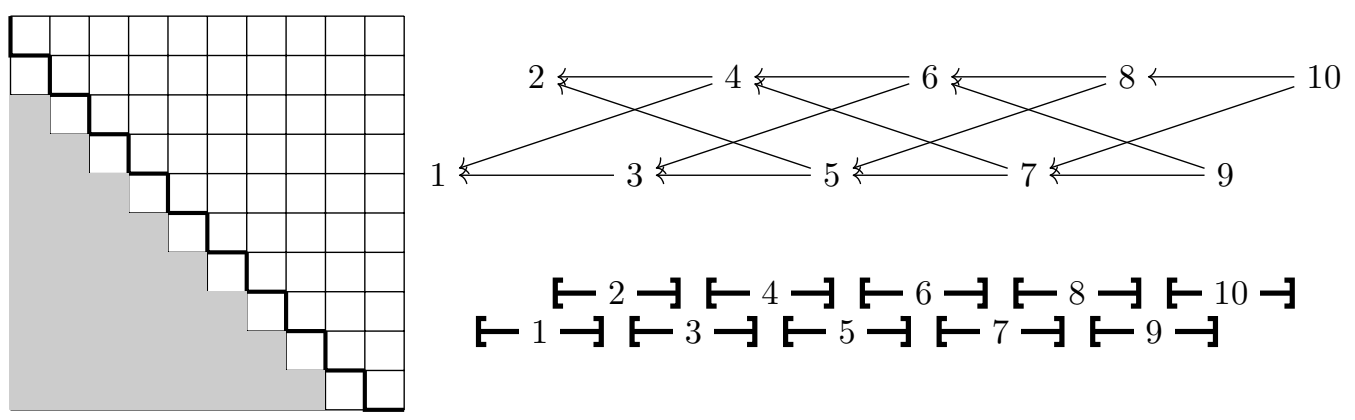

Figure 5. $\mathcal{P}_{\text {Stair(9),10 }}$ : a ladder of size 10

(1) $\left\{y_{i}, \ldots, y_{j}\right\}$ is a ladder in $\mathcal{P}$ for any $1 \leq i \leq j \leq k$.

(2) $y_{i} \leftrightarrow-\mathcal{P} y_{i+1}$ for $i \in[1, k-1]$.

(3) $y_{i} \leftarrow \mathcal{P} y_{j}$ if $j-i \geq 2$.

(4) If $x \leftarrow_{\mathcal{P}} y_{1}$ and $x \leftarrow_{\mathcal{P}} y_{2}$, then $\left\{x, y_{1}, \ldots, y_{k}\right\}$ is a ladder in $\mathcal{P}$.

(5) If $z \rightarrow_{\mathcal{P}} y_{k}$ and $z \rightarrow_{\mathcal{P}} y_{k-1}$, then $\left\{y_{1}, \ldots, y_{k}, z\right\}$ is a ladder in $\mathcal{P}$.

(6) If $y_{i}<x<y_{i+1}$ for some $i \in[1, k-1]$, then $y_{i} \leftarrow_{-\mathcal{P}} x$ and $x{ }^{--\mathcal{P}} y_{i+1}$.

Proof. It follows almost directly from the definition of a ladder and Condition ( $\pitchfork$ ).

3.5. Climbing a ladder. We define a special kind of partial orders called "ladder-climbing" orders.

Definition 3.9. For a partial order $\mathcal{P}$ on $[1, n]$, we say that someone is climbing a ladder in $\mathcal{P}$ or $\mathcal{P}$ is ladder-climbing if there exist $x, y_{1}, \ldots, y_{k} \in[1, n]$ such that

(1) $x \notin\left\{y_{1}, y_{2}, \ldots, y_{k}\right\}$,

(2) $\left\{y_{1}, y_{2}, \ldots, y_{k}\right\}$ is a ladder in $\mathcal{P}$, and

(3) $y_{1} \leftarrow \mathcal{P} x \leftarrow \mathcal{P} y_{k}$.

In this case, we also say that $x$ is climbing a ladder in $\mathcal{P}$ or $x$ is climbing (the ladder) $\left\{y_{1}, \ldots, y_{k}\right\}$ in $\mathcal{P}$. If there is no such $x$, then we say that no one is climbing a ladder in $\mathcal{P}$ or simply $\mathcal{P}$ is not ladder-climbing.

Indeed, there is a characterization of ladder-climbing partial orders in terms of the avoidance of certain suborders as the following proposition shows.

Proposition 3.10. Let $\mathcal{P}$ be a partial order on $[1, n]$. Then no one is climbing a ladder in $\mathcal{P}$ if and only if it avoids both $\mathcal{P}_{(3,1,1), 5}$ and $\mathcal{P}_{(4,2,1,1), 6}$. (See Figure 6 for the Hasse diagrams of these two orders.)

Proof. Direct calculation shows that 3 is climbing the ladder $\{1,2,4,5\}$ in $\mathcal{P}_{(3,1,1), 5}$ and 4 is climbing the ladder $\{1,2,4,5,6\}$ in $\mathcal{P}_{(4,2,1,1), 6}$. Thus if no one is climbing a ladder in $\mathcal{P}$ then it should avoid $\mathcal{P}_{(3,1,1), 5}$ and $\mathcal{P}_{(4,2,1,1), 6}$.

It remains to show that if $\mathcal{P}$ is ladder-climbing then there is a set $X \in[1, n]$ such that $\left.\mathcal{P}\right|_{X}$ is isomorphic to either $\mathcal{P}_{(3,1,1), 5}$ or $\mathcal{P}_{(4,2,1,1), 6}$. Suppose that $x$ is climbing the ladder $\left\{y_{1}, y_{2}, \ldots, y_{k}\right\}$ in $\mathcal{P}$ where $y_{1}<y_{2}<\cdots<y_{k}$, in which case $y_{1} \leftarrow \mathcal{P} x \leftarrow \mathcal{P} y_{k}$. Then without loss of generality we may assume that $y_{2} \nvdash_{\mathcal{P}} x$ and $y_{k-1} t_{\mathcal{P}} x$. Since $y_{1} \mathfrak{-}^{-\mathcal{P}} y_{2}$ and $y_{k-1} \mathfrak{-}_{\mathcal{P}} y_{k}$, this implies 
that $y_{2} \leftrightarrow-\mathcal{P} x$ and $y_{k-1} \rightarrow_{\mathcal{P}} x$ by Condition ( $\left.\pitchfork\right)$. Moreover, we have $y_{i}--_{\mathcal{P}} x$ for $i \in[2, k-1]$. Indeed, if $y_{i} \leftarrow_{\mathcal{P}} x\left(\right.$ resp. $\left.y_{i} \rightarrow_{\mathcal{P}} x\right)$ then $y_{2} \leftarrow_{\mathcal{P}} x$ (resp. $y_{k-1} \rightarrow_{\mathcal{P}} x$ ) by Condition ( $\pitchfork$ ) applied to $\left(y_{2}, y_{i}, x\right)$ (resp. $\left.\left(x, y_{i}, y_{k-1}\right)\right)$, which is a contradiction.

Since $y_{2} \leftrightarrow-\mathcal{P} x$ and $y_{k-1} \rightarrow \mathcal{P} \quad x$ we have $k \geq 4$. On the other hand, if $k \geq 7$ then $y_{2}, y_{4}, y_{6}--_{\mathcal{P}} x$ and $y_{2} \leftarrow \mathcal{P} y_{4} \leftarrow \mathcal{P} y_{6}$ which is impossible by Lemma 3.4 Thus it follows that $k \in[4,6]$. If $k=4$, then one can easily show that $\mathcal{P}$ restricted to $\left\{x, y_{1}, y_{2}, y_{3}, y_{4}\right\}$ is isomorphic to $\mathcal{P}_{(3,1,1), 5}$ where the isomorphism of posets is given by $\left(x, y_{1}, y_{2}, y_{3}, y_{4}\right) \mapsto(3,1,2,4,5)$. If $k=5$, then $\mathcal{P}$ restricted to $\left\{x, y_{1}, y_{2}, y_{3}, y_{4}, y_{5}\right\}$ is isomorphic to $\mathcal{P}_{(4,2,1,1), 6}$ where the isomorphism is given by $\left(x, y_{1}, y_{2}, y_{3}, y_{4}, y_{5}\right) \mapsto(3,1,2,4,5,6)$. Finally, if $k=6$ then $\mathcal{P}$ restricted to $\left\{x, y_{1}, y_{2}, y_{3}, y_{5}\right\}$ is isomorphic to $\mathcal{P}_{(3,1,1), 5}$, where the isomorphism is given by $\left(x, y_{1}, y_{2}, y_{3}, y_{5}\right) \mapsto(4,1,2,3,6)$. It suffices for the proof.
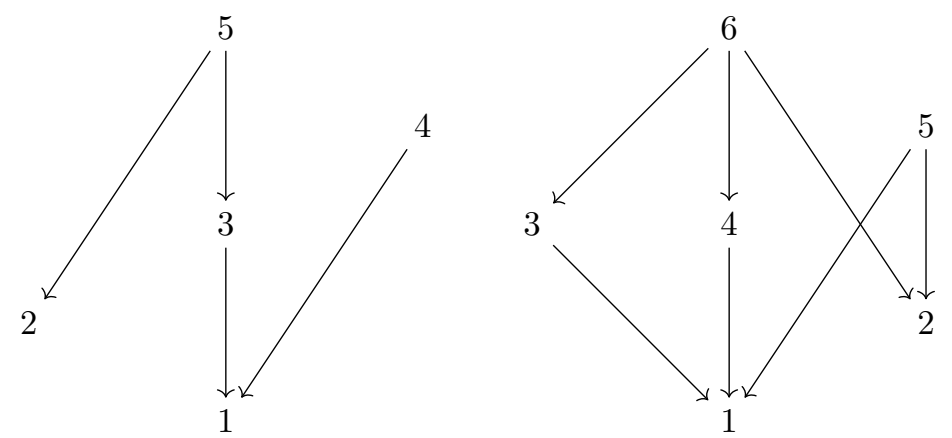

Figure 6. $\mathcal{P}_{(3,1,1), 5}$ and $\mathcal{P}_{(4,2,1,1), 6}$

There is another characterization of $\mathcal{P}_{(3,1,1), 5}$-avoiding partial orders as follows.

Lemma 3.11. The following two conditions are equivalent.

(1) $\mathcal{P}$ avoids $\mathcal{P}_{(3,1,1), 5}$

(2) "A join of two ladders is again a ladder." Suppose that $\mathcal{L}$ and $\mathcal{L}^{\prime}$ are two ladders in $\mathcal{P}$ such that $\mathcal{L} \cap \mathcal{L}^{\prime}=\{x\}$. If $x$ is the maximum in $\mathcal{L}$ and the minimum in $\mathcal{L}^{\prime}$ with respect to the usual order and $|\mathcal{L}|,\left|\mathcal{L}^{\prime}\right| \geq 3$ then $\mathcal{L} \cup \mathcal{L}^{\prime}$ is also a ladder in $\mathcal{P}$.

Proof. Suppose that $\mathcal{P}=\mathcal{P}_{(3,1,1), 5}$. Then $\{1,2,3\}$ and $\{3,4,5\}$ are ladders but $\{1,2,3,4,5\}$ is not a ladder. Thus any order that does not avoid $\mathcal{P}=\mathcal{P}_{(3,1,1), 5}$ cannot satisfy the second condition. Now suppose that $\mathcal{P}$ avoids $\mathcal{P}_{(3,1,1), 5}$ and assume that $a_{1}<\cdots<a_{k}<x<b_{1}<$ $\cdots<b_{l}$ such that $\left\{a_{1}, \ldots, a_{k}, x\right\}$ and $\left\{x, b_{1}, \ldots, b_{l}\right\}$ are ladders in $\mathcal{P}$. Then it suffices to show that $\left\{a_{k-1}, a_{k}, x, b_{1}, b_{2}\right\}$ is again a ladder in $\mathcal{P}$. By assumption we have $a_{k-1} \leftarrow_{\mathcal{P}} x \leftarrow_{\mathcal{P}} b_{2}$ and

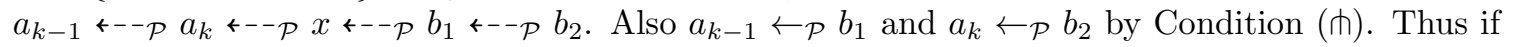
$a_{k}{ }^{--\mathcal{P}} b_{1}$ then direct calculation shows that $\mathcal{P}$ restricted to $\left\{a_{k-1}, a_{k}, x, b_{1}, b_{2}\right\}$ is isomorphic to $\mathcal{P}_{(3,1,1), 5}$, which is a contradiction. Thus $a_{k} \leftarrow_{\mathcal{P}} b_{1}$ and the result follows.

\section{4. $\mathcal{P}$-Knuth EQUivalence And the main TheOrem}

In this section we assume that a fixed natural unit interval order $\mathcal{P}$ on $[1, n]$ is given and define $\mathcal{P}$-Knuth moves and equivalences. Also we state our main theorem in this paper. 
4.1. Definition of $\mathcal{P}$-Knuth equivalences. First we define the notions of $\mathcal{P}$-Knuth moves and equivalences which generalize the ones originally introduced by Knuth.

Definition 4.1. Let $1 \leq a<b<c \leq n$ and $a \leftarrow \mathcal{P} c$. We say that two words $w, w^{\prime}$ are connected by a $\mathcal{P}$-Knuth move if they fall into one of the following situations, in which case we write $w \stackrel{\mathcal{P}}{m} w^{\prime}$ (or $w \leadsto \rightsquigarrow w^{\prime}$ if there is no confusion).

(1) If $a \leftarrow_{-\mathcal{P}} b$ and $b{ }_{--\mathcal{P}} c$, then $[\cdots b c a \cdots] \stackrel{\mathcal{P}}{\stackrel{\mathcal{P}}{m}[\cdots c a b \cdots] .}$

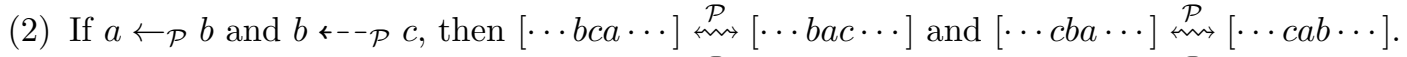

(3) If $a-_{\mathcal{P}} b$ and $b \leftarrow_{\mathcal{P}} c$, then $[\cdots b c a \cdots] \stackrel{\mathcal{P}}{\stackrel{\mathcal{P}}{m}[\cdots c b a \cdots]}$ and $[\cdots a c b \cdots] \stackrel{\mathcal{P}}{\stackrel{\mathcal{P}}{m}[\cdots c a b \cdots]}$.

(4) If $a \leftarrow_{\mathcal{P}} b$ and $b \leftarrow_{\mathcal{P}} c$, then $[\cdots b c a \cdots] \stackrel{\mathcal{P}}{\leftrightarrow}[\cdots b a c \cdots]$ and $[\cdots a c b \cdots] \stackrel{\mathcal{P}}{\leftrightarrow}[\cdots c a b \cdots]$.
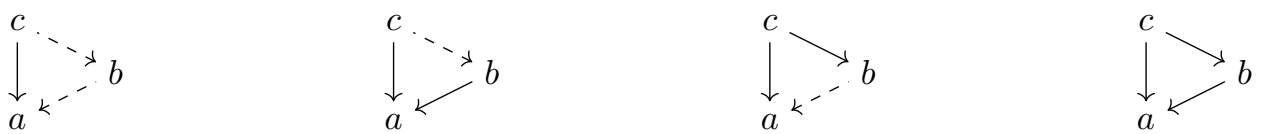

(1) $\left.\mathcal{P}\right|_{\{a, b, c\}} \simeq \mathcal{P}_{(1), 3}$

(2) $\left.\mathcal{P}\right|_{\{a, b, c\}} \simeq \mathcal{P}_{(1,1), 3}$

(3) $\left.\mathcal{P}\right|_{\{a, b, c\}} \simeq \mathcal{P}_{(2), 3}$

(4) $\left.\mathcal{P}\right|_{\{a, b, c\}} \simeq \mathcal{P}_{(2,1), 3}$

In each situation, there exists $i \in[2, n-1]$ such that the set of positions of $a, b$, and $c$ is $\{i-1, i, i+1\}$. In such a case, we say that $i$ is the position of the $\mathcal{P}$-Knuth move and also that the $\mathcal{P}$-Knuth move occurs at position $i$.

Definition 4.2. The $\mathcal{P}$-Knuth equivalence relation on the set of words is the equivalence relation generated by $\mathcal{P}$-Knuth moves. If two words $w, w^{\prime}$ are equivalent under this relation, we say that $w$ and $w^{\prime}$ are $\mathcal{P}$-Knuth equivalent and write $w \stackrel{\mathcal{P}}{\sim} w^{\prime}$. (If there is no confusion, we also say that $w$ and $w^{\prime}$ are equivalent and write $w \sim w^{\prime}$.)

Note that $\mathcal{P}$-Knuth move/equivalence revert to the usual Knuth move/equivalence when $\mathcal{P}$ is the usual order on $[1, n]$.

Example 4.3 (Figure 7). All the possible $\mathcal{P}$-Knuth moves for natural unit interval orders $\mathcal{P}$ on $[1,3]$ are described in Figure 7 Here, the underlined numbers in each word indicate its $\mathcal{P}$-descents.

4.2. Relation to $\mathcal{P}$-descents and $\mathbf{D}$ graphs. Here we relate $\mathcal{P}$-Knuth equivalences with study of dual equivalence graphs by Assaf. More precisely, we show that the graphs obtained from the $\mathcal{P}$ Knuth moves are D graphs in the sense of [AB12, Definition 4.5]. First let us discuss how $\mathcal{P}$-Knuth moves affect the $\mathcal{P}$-descents of words.

Lemma 4.4. Assume that $w, w^{\prime} \in \mathfrak{S}_{n}$ are connected by a $\mathcal{P}$-Knuth move at position $i$.

(1) We have $\left\{\operatorname{des}_{\mathcal{P}}(w) \cap\{i-1, i\}, \operatorname{des}_{\mathcal{P}}\left(w^{\prime}\right) \cap\{i-1, i\}\right\}=\{\{i-1\},\{i\}\}$.

(2) If $i>2$, then $\left\{\operatorname{des}_{\mathcal{P}}(w) \cap\{i-2, i-1\}, \operatorname{des}_{\mathcal{P}}\left(w^{\prime}\right) \cap\{i-2, i-1\}\right\}$ is equal to one of $\{\emptyset,\{i-1\}\}$, $\{\{i-2\},\{i-1\}\}$, or $\{\{i-2\},\{i-2, i-1\}\}$.

(3) If $i<n-1$, then $\left\{\operatorname{des}_{\mathcal{P}}(w) \cap\{i, i+1\}, \operatorname{des}_{\mathcal{P}}\left(w^{\prime}\right) \cap\{i, i+1\}\right\}$ is equal to one of $\{\emptyset,\{i\}\}$, $\{\{i\},\{i+1\}\}$, or $\{\{i+1\},\{i, i+1\}\}$.

(4) If $j \in[1, n-1]-[i-2, i+1]$, then $\operatorname{des}_{\mathcal{P}}(w) \cap\{j\}=\operatorname{des}_{\mathcal{P}}\left(w^{\prime}\right) \cap\{j\}$. 


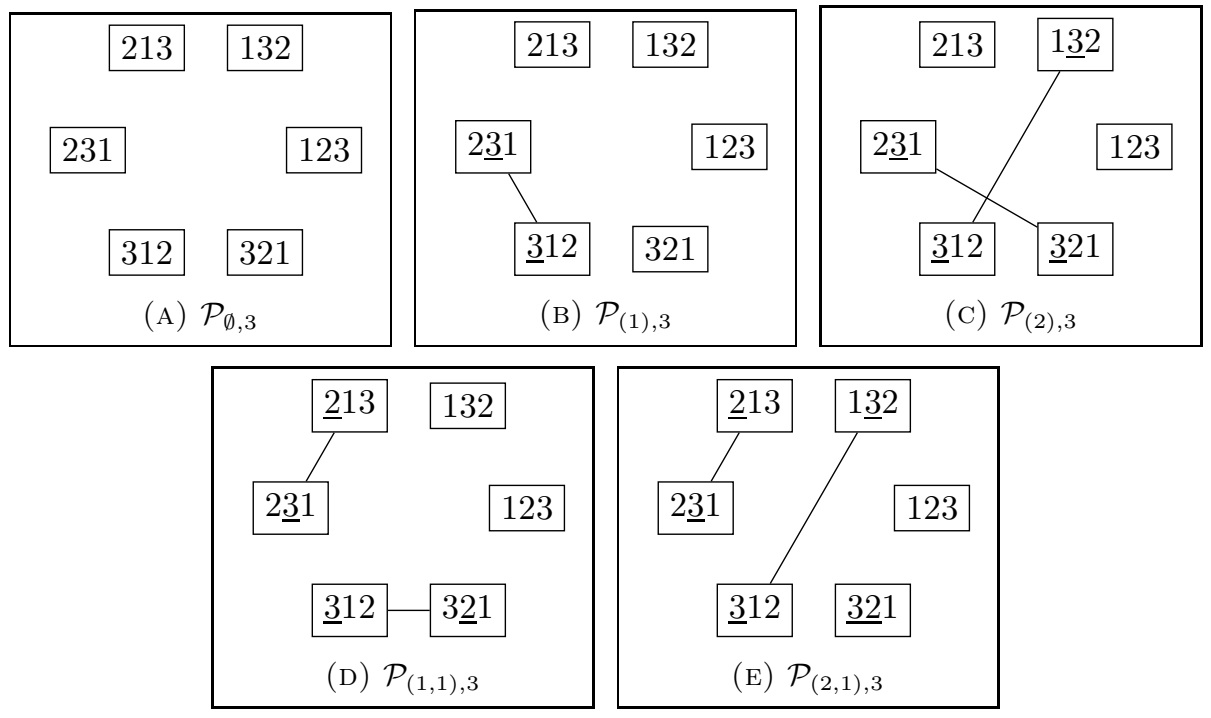

Figure 7. The $\mathcal{P}$-Knuth moves on $\mathfrak{S}_{3}$

Proof. (1) is checked case-by-case. For (2), we only need to check that $\left\{\operatorname{des}_{\mathcal{P}}(w) \cap\{i-2, i-\right.$ $\left.1\}, \operatorname{des}_{\mathcal{P}}\left(w^{\prime}\right) \cap\{i-2, i-1\}\right\} \neq\{\emptyset,\{i-2, i-1\}\}$ thanks to (1). This is also checked case-by-case. (3) is proved similarly to (2). (4) is trivial from the definition of $\mathcal{P}$-Knuth moves.

We recall the notion of signed colored graphs following [AB12, 4.2] and [Ass15, Definition 3.1].

Definition 4.5. A signed colored graph of degree $n$ is a collection $\left(V, \sigma,\left\{E_{i}\right\}_{1<i<n}\right)$ where $V$ is a set, $\sigma$ is a function $\sigma: V \rightarrow 2^{[1, n-1]}$, and each $E_{i}$ is a set of unordered pairs of different elements in $V$. (Here $2^{[1, n-1]}$ denotes the power set of $[1, n-1]$.) Each element in $V$ is called a vertex, and each element in $E_{i}$ is called an edge colored $i$.

Remark. In [AB12, 4.2] and Ass15, Definition 3.1] the function $\sigma$ assigns to each vertex $v \in V$ a sequence of length $n-1$ consisting of + and - . Their definition is equivalent to ours if we define a new sigma function, say $\sigma^{\prime}: V \rightarrow 2^{[1, n-1]}$, such that $\sigma^{\prime}(v) \ni i$ (resp. $\left.\sigma^{\prime}(v) \not \supset i\right)$ if and only if the $i$-th component of $\sigma(v)$ equals + (resp. - ).

Definition 4.6. Suppose that $V \subset \mathfrak{S}_{n}$ is closed under $\mathcal{P}$-Knuth moves. Then we define the $\mathcal{P}$ Knuth equivalence graph $\Gamma_{V}$ attached to $V$ to be $\Gamma_{V}=\left(V, \operatorname{des}_{\mathcal{P}},\left\{E_{i}\right\}_{1<i<n}\right)$ where each $E_{i}$ is the set of pairs in $V$ connected by a $\mathcal{P}$-Knuth move at position $i$.

It is clear that $\mathcal{P}$-Knuth equivalence graph is a signed colored graph of degree $n$. Now we recall the notion of D graphs following [AB12, Definition 4.2, 4.5].

Definition 4.7. A signed colored graph $\left(V, \sigma,\left\{E_{i}\right\}_{1<i<n}\right)$ of degree $n$ is called a $\mathrm{D}$ graph if the following axioms hold.

Ax1. For $w \in V$ and $1<i<m,|\sigma(w) \cap\{i-1, i\}|=1$ if and only if there exists $x \in V$ such that $\{w, x\} \in E_{i}$. Moreover, $x$ is unique when it exists.

Ax2. Whenever $\{w, x\} \in E_{i}, \sigma(w) \cap\{i\} \neq \sigma(x) \cap\{i\}$ and $\sigma(w) \cap\{h\}=\sigma(x) \cap\{h\}$ for $h \notin[i-2, i+1]$. 
Ax3. For $\{w, x\} \in E_{i}$, if $\sigma(w) \cap\{i-2\} \neq \sigma(x) \cap\{i-2\}$ then $|\sigma(w) \cap\{i-2, i-1\}|=1$. Also, if $\sigma(w) \cap\{i+1\} \neq \sigma(x) \cap\{i+1\}$ then $|\sigma(w) \cap\{i, i+1\}|=1$.

Ax5. Whenever $|i-j| \geq 3,\{w, x\} \in E_{i}$, and $\{x, y\} \in E_{j}$, there exists $v \in V$ such that $\{w, v\} \in E_{j}$ and $\{v, y\} \in E_{i}$.

We claim that the $\mathcal{P}$-Knuth equivalence graphs are indeed D graphs.

Proposition 4.8. A $\mathcal{P}$-Knuth equivalence graph is a $D$ graph.

Proof. We need to check that Ax1, Ax2, Ax3, and Ax5 hold for $\mathcal{P}$-Knuth equivalence graphs. For Ax1, it follows from the fact that the $\mathcal{P}$-Knuth move at position $i$ in Definition 4.1 occurs in all the possible cases of words satisfying $|\sigma(w) \cap\{i-1, i\}|=1$. Ax2 follows from (1) and (4) of Lemma 4.4. Ax3 follows from (2) and (3) of Lemma 4.4. Lastly, Ax5 clearly follows from the definition of $\mathcal{P}$-Knuth moves.

In [Ass15, Definition 3.2] and [AB12, Definition 4.2], they defined dual equivalence graphs which are a special kind of $\mathrm{D}$ graphs by imposing two additional axioms. This framework is used to study Schur positivity of certain quasi-symmetric functions. In particular, the "generating functions" attached to a dual equivalence graph is a single Schur function by [Ass15, Corollary 4.4]. However, our graphs are not dual equivalence graphs in general.

Example 4.9. Figure 8 shows all the connected $\mathcal{P}$-Knuth equivalence graphs on $\mathfrak{S}_{4}$ with $\mathcal{P}=$ $\mathcal{P}_{(2,1), 4}$, where underlined numbers denote $\mathcal{P}$-descents and numbers above edges indicate their colors. (Written above each connected component is the corresponding generating function which we will define in a moment.) There is one connected component with 5 vertices which satisfies neither Axiom 4 nor Axiom 6 of [Ass15, Definition 3.2] for dual equivalence graphs.

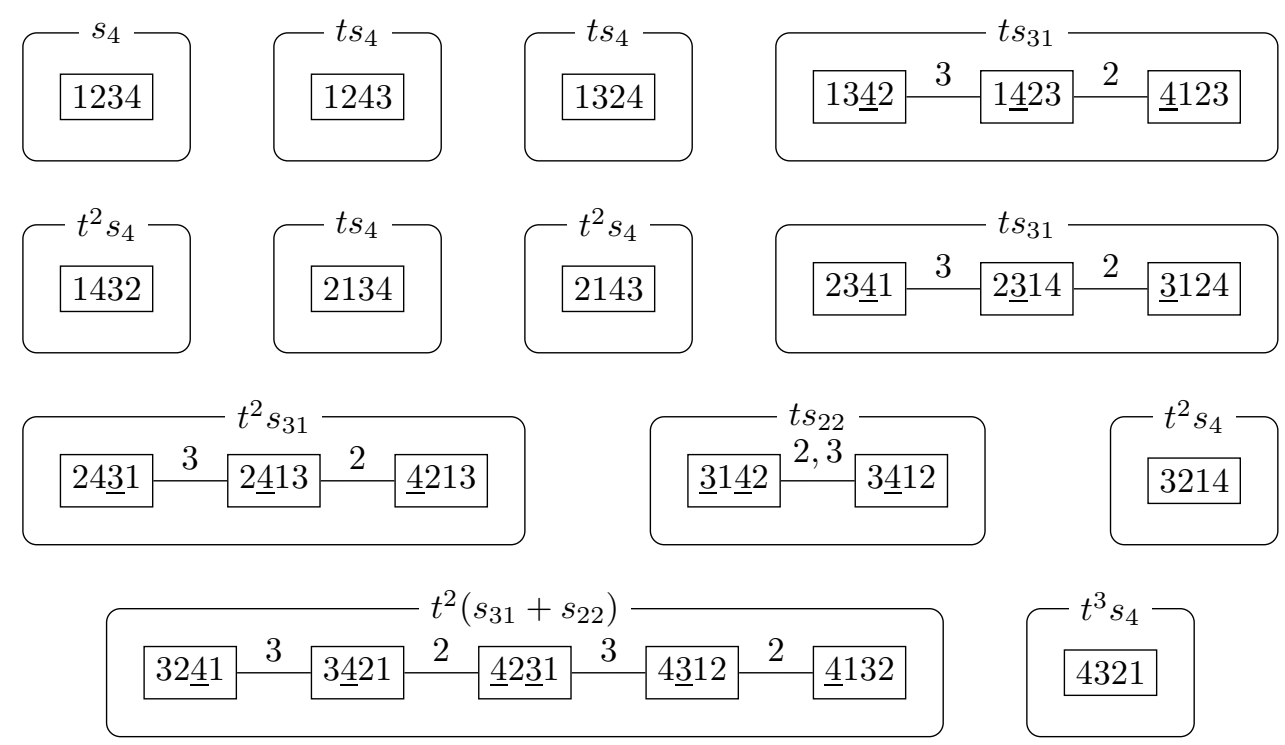

FIgURE $8 . \mathcal{P}_{(2,1), 4}$-Knuth equivalence graph 
4.3. Genuine $\mathcal{P}$-height and fake $\mathcal{P}$-inversion number. Here we prove that the $\mathcal{P}$-Knuth move preserves genuine $\mathcal{P}$-heights and fake $\mathcal{P}$-inversion numbers of permutations. For the former claim, we need to impose assumption that $\mathcal{P}$ is not ladder-climbing.

Proposition 4.10. Suppose that $\mathcal{P}$ avoids $\mathcal{P}_{(3,1,1), 5}$ and $\mathcal{P}_{(4,2,1,1), 6}$. If $w \stackrel{\mathcal{P}}{m} w^{\prime}$, then $\operatorname{g}$-ht $(w)=$ $\mathrm{g}$-ht $\left(w^{\prime}\right)$. As a result, the genuine $\mathcal{P}$-height is constant on any connected $\mathcal{P}$-Knuth equivalence graph.

The proof of this proposition will be given in Section 8

Remark. If we allow that $\mathcal{P}$ is ladder-climbing, then the above proposition is no longer true. For example, when $\mathcal{P}=\mathcal{P}_{(3,1,1), 5}$ we have $53241 \stackrel{\mathcal{P}}{\stackrel{m}{m} 53412 \text { but g-ht }}{ }_{\mathcal{P}}(53241)=|(5,3)|=2 \neq$

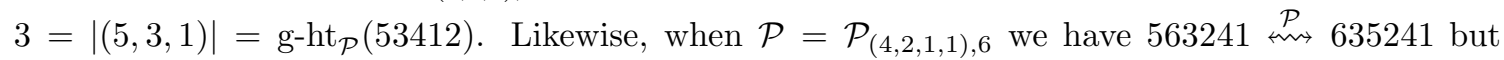
$\mathrm{g}-\mathrm{ht}_{\mathcal{P}}(563241)=|(6,4,1)|=3 \neq 2=|(6,3)|=\mathrm{g}^{-\mathrm{ht}_{\mathcal{P}}}(635241)$.

Lemma 4.11. If $w \stackrel{\mathcal{P}}{\mathrm{m}} w^{\prime}$, then $\left|\mathrm{f}-\operatorname{inv}_{\mathcal{P}}(w)\right|=\left|\mathrm{f}-\operatorname{inv}_{\mathcal{P}}\left(w^{\prime}\right)\right|$. As a result, the fake $\mathcal{P}$-inversion number is constant on any connected $\mathcal{P}$-Knuth equivalence graph.

Proof. Suppose that the $\mathcal{P}$-Knuth move $w \stackrel{\mathcal{P}}{m} w^{\prime}$ occurs at position $i$. If either $x \notin\left\{w_{i-1}, w_{i}, w_{i+1}\right\}$ or $y \notin\left\{w_{i-1}, w_{i}, w_{i+1}\right\}$ then it is clear that $\operatorname{finv}_{\mathcal{P}}(w) \cap\{(x, y)\}=\mathrm{f}_{-\operatorname{inv}_{\mathcal{P}}}\left(w^{\prime}\right) \cap\{(x, y)\}$ since the relative position of $x$ and $y$ does not change. Thus for the verification of this lemma we may restrict our attention to words of length 3, e.g. Figure 7 Now the lemma follows from case-by-case observation.

4.4. Generating functions and the main theorem. Let us define a generating function of a $\mathcal{P}$-Knuth equivalence graph. (cf. [SW16, Theorem 3.1])

Definition 4.12. For a $\mathcal{P}$-Knuth equivalence graph $\Gamma_{V}=\left(V, \operatorname{des}_{\mathcal{P}},\left\{E_{i}\right\}\right)$, its generating function is defined to be $\gamma_{V}:=\sum_{w \in V} t^{\left|\mathrm{f}-\operatorname{inv}_{\mathcal{P}}(w)\right|} F_{\operatorname{des}_{\mathcal{P}}(w)}$.

If we consider a connected $\mathcal{P}$-Knuth equivalence graph, then we may factor out $t^{|f-i n v \mathcal{P}(w)|}$ from the formula due to Lemma 4.11. More precisely, if $\Gamma_{V}$ is a connected graph then $\gamma_{V}:=$ $t^{\left|\mathrm{f}-\operatorname{inv}_{\mathcal{P}}(V)\right|} \sum_{w \in V} F_{\operatorname{des}_{\mathcal{P}}(w)}$ where $\left|\mathrm{f}_{\text {-inv }} \mathcal{P}(V)\right|$ is the fake $\mathcal{P}$-inversion number of any element in $V$. Now we state the main theorem of this paper. Its proof is given in Section 9 Note that this strengthens [Gas96. Theorem 4] for a natural unit interval order which avoids $\mathcal{P}_{(3,1,1), 5}$ and $\mathcal{P}_{(4,2,1,1), 6}$.

Theorem 4.13 (Main theorem). Suppose that $\mathcal{P}$ is a natural unit interval order on $[1, n]$ which avoids $\mathcal{P}_{(3,1,1), 5}$ and $\mathcal{P}_{(4,2,1,1), 6}$. Let $\Gamma=\left(V, \operatorname{des}_{\mathcal{P}},\left\{E_{i}\right\}\right)$ be a connected $\mathcal{P}$-Knuth equivalence graph and $\gamma_{V}$ be its generating function. Let $w_{1}, \ldots, w_{k}$ be all the elements in $V$ each of which is the reading word of the $\mathcal{P}$-tableau $P T_{1}, \ldots, P T_{k}$, of shape $\lambda_{1}, \ldots, \lambda_{k}$, respectively. Then we have $\gamma_{V}=$ $t^{\left|\mathrm{f}-\operatorname{inv}_{\mathcal{P}}(V)\right|}\left(s_{\lambda_{1}}+\cdots+s_{\lambda_{k}}\right)$, where $\mid \mathrm{f}$-inv $\mathcal{P}_{\mathcal{P}}(V) \mid$ is the fake $\mathcal{P}$-inversion number of any element in $V$. Furthermore, we have $l\left(\lambda_{1}\right)=\cdots=l\left(\lambda_{k}\right)$ which is also equal to the genuine $\mathcal{P}$-height of any $w \in V$.

Conjecture 4.14 (Main conjecture). The claim of the Theorem 4.13 is true for all unit interval orders $\mathcal{P}$ (except the last sentense).

See Section 7.1 for some examples of $\mathcal{P}$-Knuth equivalence graphs and their generating functions. The following corollary is a direct consequence, which generalizes both [Gas96. Theorem 3] and [SW16, Theorem 6.3] for a natural unit interval order which avoids $\mathcal{P}_{(3,1,1), 5}$ and $\mathcal{P}_{(4,2,1,1), 6}$. 
Corollary 4.15. Suppose that $\mathcal{P}$ is a natural unit interval order on $[1, n]$ which avoids $\mathcal{P}_{(3,1,1), 5}$ and $\mathcal{P}_{(4,2,1,1), 6}$. Then the generating function of any $\mathcal{P}$-Knuth equivalence graph is Schur positive, i.e. it is a symmetric function and its coefficients with respect to the expansion of Schur functions are polynomials in $t$ with nonnegative integer coefficients.

\section{Column insertion Algorithm}

In this section, we assume that a fixed natural unit interval order $\mathcal{P}$ is given and define a column insertion algorithm.

5.1. Column insertion algorithm $\mathbf{A l g}_{\Phi}$. For convenience, we add $\infty,-\infty$ to the poset $([1, n], \mathcal{P})$ so that $\infty \rightarrow_{\mathcal{P}} i$ (resp. $-\infty \leftarrow_{\mathcal{P}} i$ ) for any $i \in[1, n]$. We define

$$
\begin{aligned}
\mathfrak{A} & :=\left\{\left(a_{m}, \ldots, a_{1}\right) \mid m \in \mathbb{N}, a_{i} \in[1, n] \cup\{\infty\}, a_{i} \neq a_{j} \text { if } i \neq j \text { and } a_{i}, a_{j} \neq \infty\right\}, \\
\mathfrak{C} & :=\left\{\left(c_{l}, \ldots, c_{1}\right) \mid l \in \mathbb{N}, c_{i} \in[1, n], c_{i} \leftarrow \mathcal{P} c_{j} \text { if } i<j\right\}, \\
\mathfrak{A C}: & :=\left\{(\alpha, c) \in \mathfrak{A} \times \mathfrak{C} \mid a_{i} \neq c_{j} \text { for any } i, j\right\}, \text { and } \\
\mathfrak{C A} & :=\left\{(c, \alpha) \in \mathfrak{C} \times \mathfrak{A} \mid a_{i} \neq c_{j} \text { for any } i, j\right\} .
\end{aligned}
$$

One may regard $\mathfrak{A}$ as a set of (input/output) words and $\mathfrak{C}$ as a set of chains, i.e. one-column $\mathcal{P}$-tableaux. (Recall that we read columns from bottom to top.)

We introduce the column insertion algorithm $\mathbf{A l g}_{\Phi}$. This defines a function $\Phi: \mathfrak{A C} \rightarrow \mathfrak{C A}$ and is described in terms of the pseudocode Algorithm 1 .

Let us investigate this algorithm in more detail. It takes the input $(\alpha, c) \in \mathfrak{A C}$ where $\alpha=$ $\left(a_{m}, \ldots, a_{1}\right)$ and $c=\left(c_{l}, \ldots, c_{1}\right)$. Initiate $\mathfrak{b}=\left(b_{m}, \ldots, b_{1}\right)$ with $\left(a_{m}, \ldots, a_{1}\right)$ and $\mathfrak{d}=\left(d_{l}, \ldots, d_{1}\right)$ with $\left(c_{l}, \ldots, c_{1}\right)$. (Here $l=|\mathfrak{d}|$, which may change as the algorithm is performed.) Also we set $d_{0}:=-\infty$ to simplify our argument. Initialize $p$ with 1.

5.1.1. If $p>m=|\alpha|$, then we terminate the algorithm and return $(d, \beta)=(\mathfrak{d}, \mathfrak{b})$.

5.1.2. Case $\infty(\mathrm{a})$. Suppose that $a_{p}=\infty$. Then we increase $p$ by 1 and repeat the algorithm from the beginning.

Remark. The reason why we call it Case $\infty$ (a) (instead of Case $\infty$ ) shall become apparent when we describe another algorithm $\Psi_{X}$ in the proof of Proposition 5.6 .

From now on we suppose $a_{p} \neq \infty$ and choose $r \in[0, l]$ such that $d_{r}<a_{p}<d_{r+1}$. (If $d_{l}<a_{p}$, then we set $r=l$.) First suppose that $a_{p}$ and $d_{r}$ are comparable, i.e. $d_{r} \leftarrow \mathcal{P} a_{p}$.

5.1.3. Case I(a). We first consider the case when $r=l$, i.e. $a_{p}$ is bigger than any element in $\mathfrak{d}$ with respect to $\mathcal{P}$. (This includes the case when $r=l=0$, i.e. $\mathfrak{d}$ is an empty chain.) In this case we "add $a_{p}$ to the end of the chain $\mathfrak{d}$ ", i.e. set $d_{l+1}:=a_{p}$ and replace $\mathfrak{d}$ with $\left(d_{l+1}=a_{p}, d_{l}, \cdots, d_{1}\right)$. (As a result, the length of $\mathfrak{d}$ increases by 1.) After this, we increase $p$ by 1 and repeat the algorithm from the beginning. 


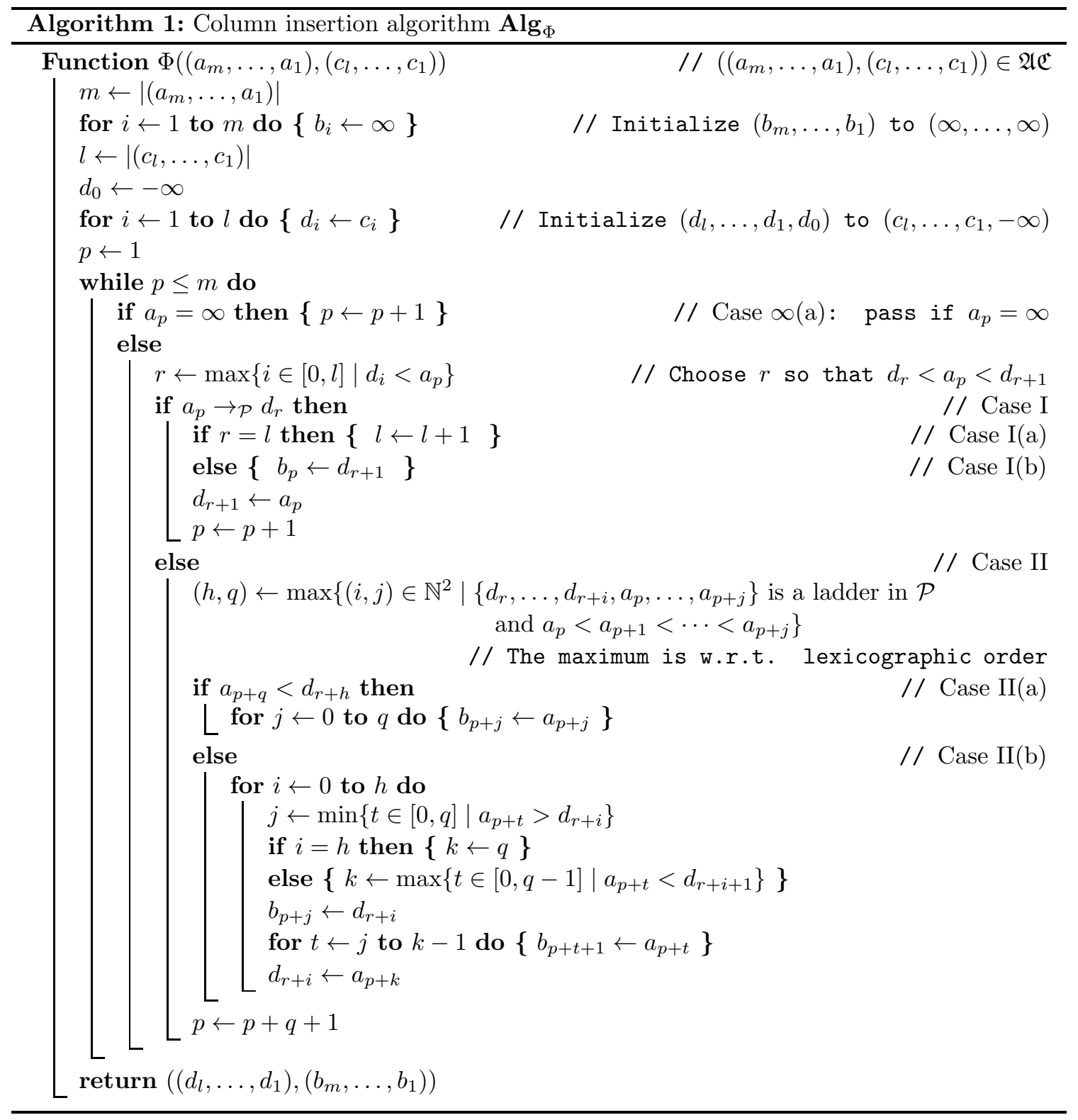

5.1.4. Case I(b). Now suppose that $r<l$. (This include the case when $-\infty=d_{0}<a_{p}<d_{1}$.) Then " $a_{p}$ bumps $d_{r+1}$ "; we set $b_{p}:=d_{r+1}$ and then replace $d_{r+1}$ in $\mathfrak{d}$ with $a_{p}$. For example, if $1 \leftarrow_{\mathcal{P}} 3$ and $1 \leftarrow_{\mathcal{P}} 2$, then $\Phi((2),(3,1))=((2,1),(3))$. (Whether $2 \leftarrow_{\mathcal{P}} 3$ or not does not affect the result here.) After this, we increase $p$ by 1 and repeat the algorithm from the beginning.

Now we suppose that $a_{p}$ and $d_{r}$ are not comparable (which forces that $r>0$ ). We set

$A=\left\{(i, j) \in \mathbb{N}^{2} \mid a_{p}<a_{p+1}<\cdots<a_{p+j}\right.$ and $\left\{d_{r}, \ldots, d_{r+i}, a_{p}, \ldots, a_{p+j}\right\}$ is a ladder in $\left.\mathcal{P}\right\}$. 
Note that $(0,0) \in A$ as $\left\{d_{r}, a_{p}\right\}$ is a ladder in $\mathcal{P}$ by assumption (since $\mathcal{P}_{\operatorname{Stair}(1), 2}=\mathcal{P}_{\emptyset, 2}$ ). We set $(h, q)$ to be the maximum of $A$ with respect to lexicographic order. In other words, we choose $(h, q)$ such that

- $a_{p}<a_{p+1}<\cdots<a_{p+q}$ with respect to the usual order,

- $\left\{d_{r}, \ldots, d_{r+h}, a_{p}, \ldots, a_{p+q}\right\}$ is a ladder in $\mathcal{P}$,

- $h$ is the biggest among such possible $(h, q)$ 's, and

- $q$ is the biggest among such possible $(h, q)$ 's with $h$ chosen above.

For later use, we define:

Definition 5.1. We assume the situation above. Then the phrase "maximality in Case II" indicates the maximality of $(h, q)$ in $A$.

5.1.5. Case II(a). First we suppose that $a_{p+q}<d_{r+h}$, i.e. $\max \left\{d_{r}, \ldots, d_{r+h}, a_{p}, \ldots, a_{p+q}\right\}=d_{r+h}$. (e.g. Example[7.1 and 7.3) In this case we do not alter the chain $\mathfrak{d}$ and simply let $a_{p}, \ldots, a_{p+q}$ "pass through the chain", i.e. set $b_{i}:=a_{i}$ for $i \in[p, p+q]$. After this, we increase $p$ by $q+1$ and repeat the algorithm from the beginning.

Remark. Here, the maximality in Case II means that either $p+q=m$ or $a_{p+q+1}$ does not satisfy both $a_{p+q+1} \rightarrow_{\mathcal{P}} a_{p+q}$ and $a_{p+q+1} \rightarrow_{\mathcal{P}} d_{r+h}$.

5.1.6. Case II(b). The remaining case is when $a_{p+q}>d_{r+h}$, i.e. $\max \left\{d_{r}, \ldots, d_{r+h}, a_{p}, \ldots, a_{p+q}\right\}=$ $a_{p+q}$. (e.g. Example 7.2 and 7.3) We set $p-1=u(r-1)<u(r)<u(r+1)<\cdots<u(r+h)=p+q$ such that $d_{i}<a_{u(i-1)+1}<\cdots<a_{u(i)}$ for $i \in[r, r+h]$. Then we replace $d_{r}, \ldots, d_{r+h}$ on the chain in $\mathfrak{d}$ with $a_{u(r)}, \ldots, a_{u(r+h)}$, respectively. Furthermore, for $j \in[p, p+q]$ we set

$$
b_{j}:=\left\{\begin{array}{lr}
d_{i} & \text { if } j=u(i-1)+1 \text { for some } i \in[r, r+h], \\
a_{j-1} & \text { otherwise. }
\end{array}\right.
$$

After this, we increase $p$ by $q+1$ and repeat the algorithm from the beginning.

Remark. Here, the maximality in Case II means that

- either $p+q=m$ or $a_{p+q+1}$ does not satisfy both $a_{p+q+1} \rightarrow_{\mathcal{P}} a_{p+q}$ and $a_{p+q+1} \rightarrow_{\mathcal{P}} a_{p+q}$ (or $a_{p+q+1} \rightarrow \mathcal{P} d_{r+h}$ if $a_{p+q}-\rightarrow \mathcal{P} d_{r+h}$ ), and

- either $r+h=l$ or $d_{r+h+1} \quad f_{\rightarrow \mathcal{P}} a_{i}$ for any $i \in[p, p+q]$.

One may check that the second condition is equivalent to

- either $r+h=l$ or $d_{r+h+1} \rightarrow_{\mathcal{P}} a_{i}$ for any $i \in[p, p+q]$.

For later use, we define:

Definition 5.2. We say that $a_{p}$ is in Case I(a), 1(b), etc. if the step in the column insertion algorithm $\mathbf{A} \lg _{\Phi}$ processing $a_{p}$ corresponds to Case I(a), 1(b), etc.

This finishes the description of the algorithm $\mathbf{A l g}_{\Phi}$. See Section 7.2 for some examples about this algorithm. Before we proceed, we need to check that:

Theorem 5.3. The algorithm $\mathbf{A l g}_{\Phi}$ is well-defined, i.e. $\Phi(\alpha, c) \in \mathfrak{C A}$. 
Proof. First suppose that $a_{1}, a_{m} \neq \infty$ and only one step of $\mathbf{A l g}_{\Phi}$ is performed when calculating $\Phi\left(\left(a_{m}, \ldots, a_{1}\right),\left(c_{l}, \ldots, c_{1}\right)\right)=\left(\left(d_{l^{\prime}}, \ldots, d_{1}\right),\left(b_{m}, \ldots, b_{1}\right)\right)$. Then we need to show that $b_{i}, d_{j}$ for $i \in[1, m], j \in\left[1, l^{\prime}\right]$ are pairwise different (possibly except $\infty$ ) and $d_{1} \leftarrow \mathcal{P} d_{2} \leftarrow \mathcal{P} \cdots \leftarrow \mathcal{P} d_{l^{\prime}}$. But the first part is clear from the assumption that $a_{1}, \ldots, a_{m}, c_{1}, \ldots, c_{l}$ are pairwise different. The second part is also easily checked case-by-case using Condition ( $\pitchfork)$. Now the statement in the general case follows from induction on the number of steps.

5.2. Properties of $\mathbf{A l g}_{\Phi}$. Here we discuss some properties of $\mathbf{A} \lg _{\Phi}$. Firstly, if $\Phi(\alpha, c)=(d, \beta)$ then it is easy to observe the following. (We will use these facts without reminder later on.)

- if $a_{i}$ is in Case $\infty(\mathrm{a})$ or Case $\mathrm{I}(\mathrm{a})$, we have $b_{i}=\infty$,

- if $a_{i}$ is in Case $\mathrm{I}(\mathrm{b})$, we have $a_{i}<b_{i} \neq \infty$,

- if $a_{i}$ is in Case II(a), we have $a_{i}=b_{i}$, and

- if $a_{i}$ is in Case II(b), we have $a_{i} \rightarrow \mathcal{P} b_{i}$.

The following lemma is less trivial.

Lemma 5.4. For $\alpha=\left(a_{m}, \ldots, a_{1}\right)$ and $c=\left(c_{l}, \ldots, c_{1}\right)$, suppose that there exists $i \in[1, l]$ such that $c_{i} \leftarrow \mathcal{P} a_{j}$ for all $j \in[1, m]$. If $\Phi(\alpha, c)=\left(\left(d_{l^{\prime}}, \ldots, d_{1}\right),-\right)$, then we have $c_{j}=d_{j}$ for $j \in[1, i]$.

Proof. It is shown by case-by-case observation.

The proofs of the following two propositions are provided in Section 8

Proposition 5.5. Suppose that $\Phi(\alpha, c)=(d, \beta)$ where $\alpha=\left(a_{m}, \ldots, a_{1}\right), \beta=\left(b_{m}, \ldots, b_{1}\right)$, and $c=\left(c_{l}, \ldots, c_{1}\right)$. Write $\alpha^{f}$ (resp. $\left.\beta^{f}\right)$ to be the word obtained by removing $\infty$ from $\alpha$ (resp. $\left.\beta\right)$.

(A) $\alpha^{f}+c$ and $d+\beta^{f}$ are $\mathcal{P}$-Knuth equivalent. In particular, $\alpha^{f}+c=d+\beta^{f}$ as sets.

(B) Suppose that $\alpha \in \mathfrak{C}, m \geq l$, and $(\alpha, c)$ satisfies the $\mathcal{P}$-tableau condition, i.e. $a_{i} \nrightarrow_{\mathcal{P}} c_{i}$ for $i \in[1, l]$. Then $d=\alpha$ and $\beta=(\infty, \ldots, \infty)+c$.

(C) If $a_{i}, a_{i+1} \neq \infty$ and $a_{i} \leftarrow_{\mathcal{P}} a_{i+1}$, then either $\left[b_{i+1}=\infty\right]$ or $\left[b_{i}, b_{i+1} \neq \infty\right.$ and $\left.b_{i} \leftarrow_{\mathcal{P}} b_{i+1}\right]$.

(D) If $a_{i}, a_{i+1} \neq \infty$ and $a_{i} \nvdash_{\mathcal{P}} a_{i+1}$, then either $\left[b_{i}=\infty, b_{i+1} \neq \infty\right]$ or $\left[b_{i}, b_{i+1} \neq \infty\right.$ and $\left.b_{i} \psi_{\mathcal{P}} b_{i+1}\right]$.

Proposition 5.6. Suppose that $\left(\alpha=\left(a_{m}, \ldots, a_{1}\right), c\right),\left(\alpha^{\prime}=\left(a_{m}^{\prime}, \ldots, a_{1}^{\prime}\right), c^{\prime}\right) \in \mathfrak{A C}$ satisfy $\Phi(\alpha, c)=$ $\Phi\left(\alpha^{\prime}, c^{\prime}\right)$ and $a_{i}=\infty \Leftrightarrow a_{i}^{\prime}=\infty$. Then we have $(\alpha, c)=\left(\alpha^{\prime}, c^{\prime}\right)$.

5.3. Another algorithm $\mathbf{A l g}_{\Psi}$. Here we introduce another column insertion algorithm $\mathbf{A} \mathbf{l g}_{\Psi}$ which resembles $\mathbf{A l g}_{\Phi}$. This will not be used for the definition of the $\mathcal{P}$-Robinson-Schensted algorithm in the next section, but it will play an important role when we prove Proposition 5.6. Also see Section 7.2 for some examples about this algorithm.

For a subset $X \subset \mathbb{Z}_{>0}$, the algorithm $\operatorname{Alg}_{\Psi}$ defines a function $\Psi_{X}: \mathfrak{A C} \rightarrow \mathfrak{C A}$ and is described by the pseudocode Algorithm 2 . Note that the only difference between $\mathbf{A} \mathbf{l g}_{\Phi}$ and $\mathbf{A} \mathbf{l g}_{\Psi}$ is when $a_{p}=\infty, p \in X$, and $\mathfrak{d} \neq \emptyset$, which is as follows.

Case $\infty(\mathrm{b})$ Suppose that $a_{p}=\infty, p \in X$, and $\mathfrak{d} \neq \emptyset$. Then we "drag the first entry of $\mathfrak{d}$ to $\mathfrak{b}$ ", i.e. ${\overline{\text { set }} b_{p}:=d_{1}}_{1}$ and replace $\mathfrak{d}$ with $\left(d_{l}, \ldots, d_{2}\right)$. (As a result, the length of $\mathfrak{d}$ decreases by 1.) After this, we increase $p$ by 1 and repeat the algorithm from the beginning.

Indeed, if $X=\emptyset$ then $\mathbf{A l g}_{\Psi}$ and $\Psi_{X}$ revert to $\mathbf{A l g}_{\Phi}$ and $\Phi$, respectively. 


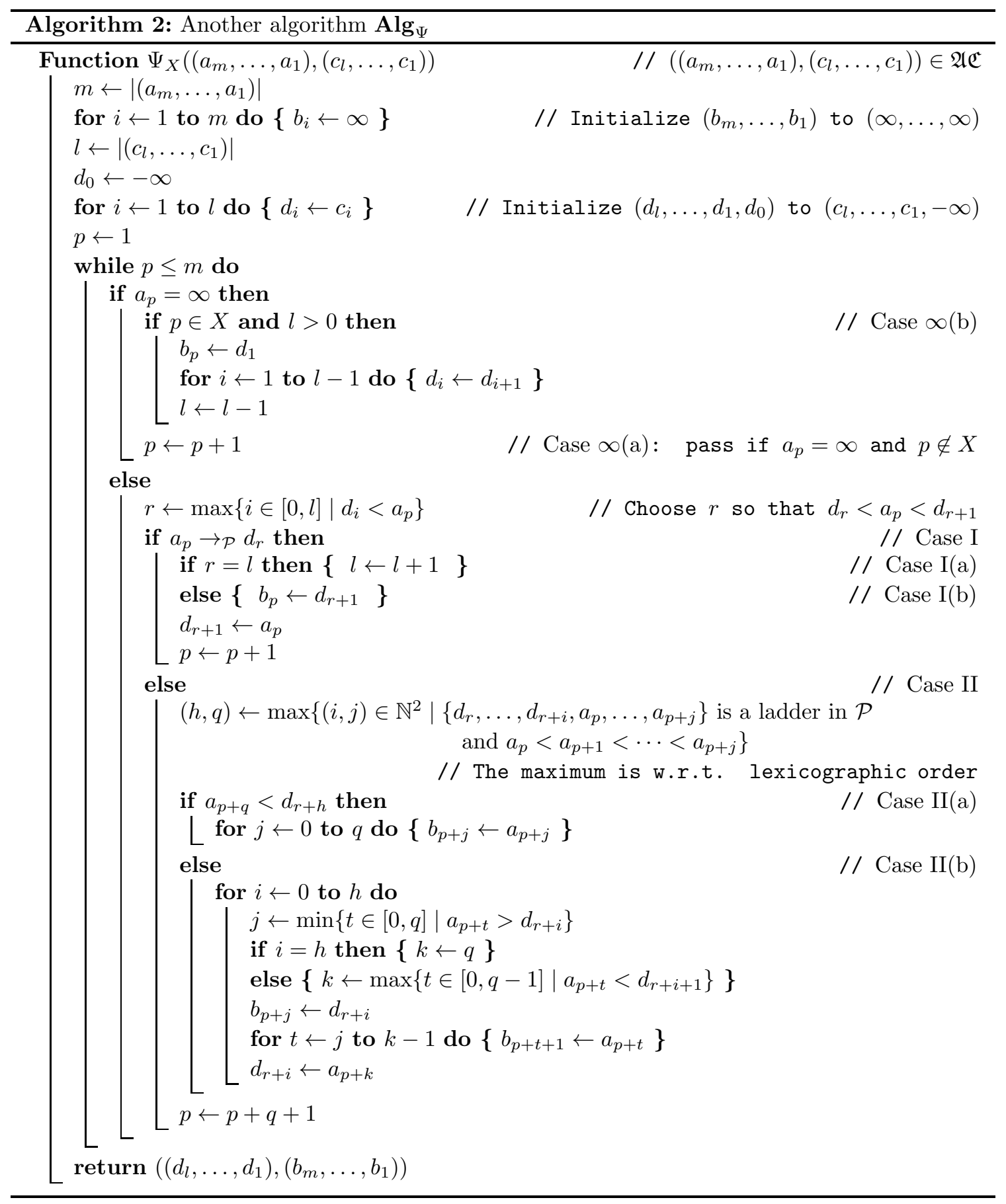


Lemma 5.7. The algorithm $\Psi_{X}$ is well-defined.

Proof. It is proved in the same manner as Theorem 5.3 .

\section{6. $\mathcal{P}$-Robinson-Schensted ALgorithm}

In this section, we assume that a fixed natural unit interval order $\mathcal{P}$ is given and define a $\mathcal{P}$-Robinson-Schensted algorithm.

6.1. $\mathcal{P}$-Robinson-Schensted algorithm. We identify $\mathcal{P}$-Tab with the subset of $\mathfrak{C}^{n}$ such that $\left(c^{1}, c^{2}, \ldots, c^{n}\right) \in \mathfrak{C}^{n}$ corresponds to the $\mathcal{P}$-tableau whose reading word is $c^{1}+c^{2}+\cdots+c^{n}$ if such a $\mathcal{P}$-tableau exists. We define the $\mathcal{P}$-Robinson-Schensted algorithm $\mathbf{A} \lg _{\mathcal{P} \text {-RS }}$ as in Algorithm 3 ,

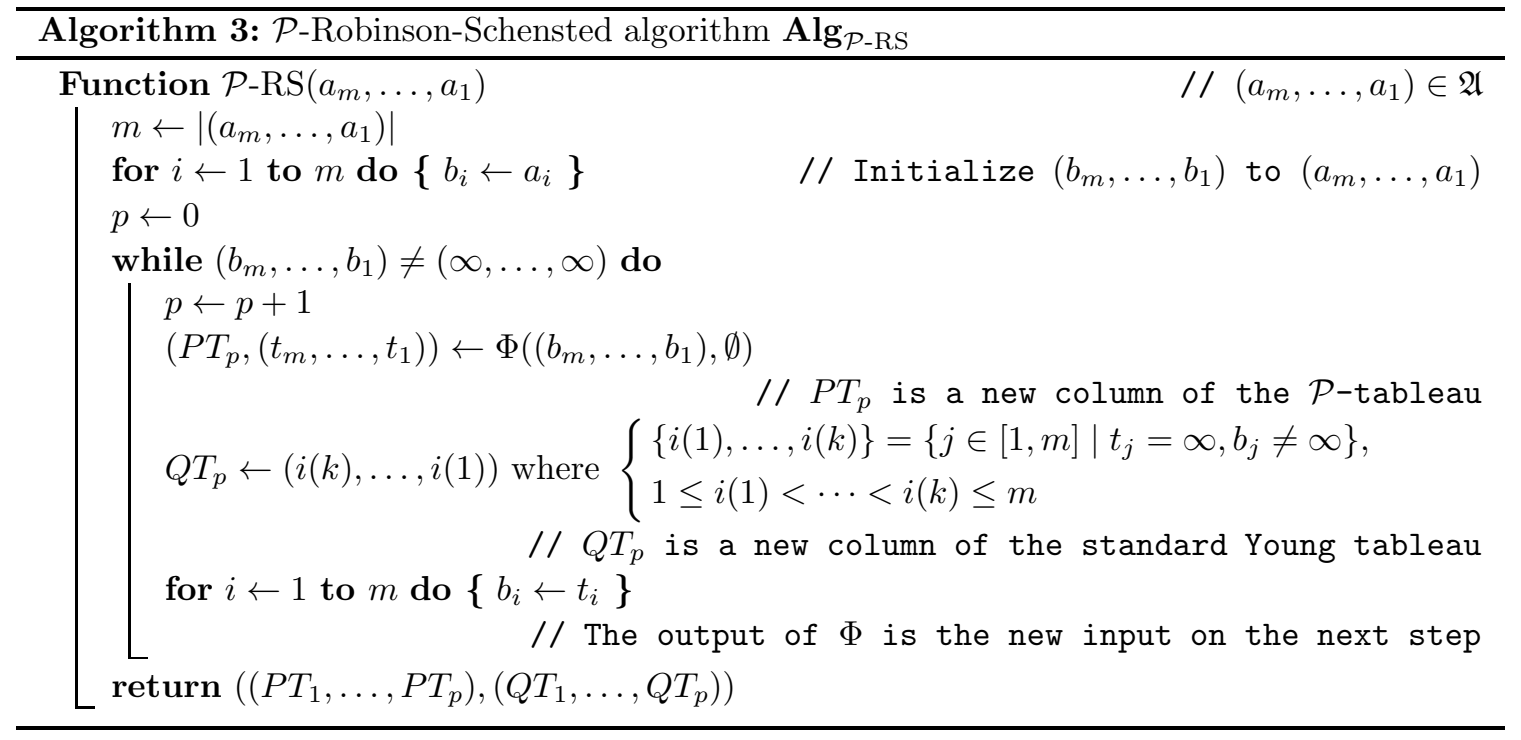

Let us describe the algorithm in detail. This algorithm takes an input $\alpha=\left(a_{m}, \ldots, a_{1}\right) \in \mathfrak{A}$ and produces an output $(P T, Q T)$. Initialize $p$ with 0 and $\left(b_{m}, \ldots, b_{1}\right)$ with $\left(a_{m}, \ldots, a_{1}\right)$.

6.1.1. If $\left(b_{m}, \ldots, b_{1}\right)=(\infty, \ldots, \infty)$ then terminate the algorithm and return $(P T, Q T)$ where $P T=\left(P T_{1}, \ldots, P T_{p}\right)$ and $Q T=\left(Q T_{1}, \ldots, Q T_{p}\right)$.

6.1.2. Otherwise, we increase p by 1 and set $\left(P T_{p},\left(t_{m}, \ldots, t_{1}\right)\right)$ to be $\Phi\left(\left(b_{m}, \ldots, b_{1}\right), \emptyset\right)$. Also we set $Q T_{p}=(i(k), \ldots, i(1))$ where $i(1)<\cdots<i(k)$ are chosen such that $\{i(1), \ldots, i(k)\}=\{j \in$ $\left.[1 . m] \mid t_{j}=\infty, b_{j} \neq \infty\right\}$, i.e. they are indices where Case $\mathrm{I}(\mathrm{a})$ of $\mathbf{A l g}_{\Phi}$ occured in the calculation of $\Phi\left(\left(b_{m}, \ldots, b_{1}\right), \emptyset\right)$. After this, we set $\left(b_{m}, \ldots, b_{1}\right)$ to be $\left(t_{m}, \ldots, t_{1}\right)$ and repeat the algorithm from the beginning.

This finishes the description of the algorithm $\mathbf{A l g}_{\mathcal{P} \text {-RS }}$. It is clear that each column of $P T$ (resp. $Q T$ ) is a chain with respect to $\mathcal{P}$ (resp. the usual order). However, it is not clear at this moment that PT (resp. $Q T$ ) is a $\mathcal{P}$-tableau (standard Young tableau). Indeed, it is not always so; see 
Section 7.4 for such examples. However, we will observe that this algorithm behaves well when the given partial order on $[1, n]$ avoids $\mathcal{P}_{(3,1,1), 5}$ and $\mathcal{P}_{(4,2,1,1), 6}$.

6.2. Properties of $\mathcal{P}$-RS. Let $T_{\lambda} \in \mathrm{SYT}_{\lambda}$ be the standard Young tableau of shape $\lambda$ where $\lambda^{\prime}=$ $\left(l_{1}, l_{2}, \ldots, l_{p}\right)$ such that the $i$-th column of $T_{\lambda}$ consists of $\left(\sum_{k=1}^{i-1} l_{k}\right)+1,\left(\sum_{k=1}^{i-1} l_{k}\right)+2, \ldots,\left(\sum_{k=1}^{i} l_{k}\right)$. For example, we have $T_{(4,3,1)}=$\begin{tabular}{|l|l|l|l|l|}
1 & 4 & 6 & 8 \\
\hline 2 & 5 & 7
\end{tabular} . The following theorem summarizes important properties \begin{tabular}{l|l|l|l|l|l}
\hline & 5 & 7 \\
\hline & & & -1 \\
\hline
\end{tabular}

of the algorithm $\mathbf{A} \lg _{\mathcal{P}-\mathrm{RS}}$ which is proved in Section 9, together with the main theorem (Theorem 4.13) of this paper.

Theorem 6.1. Suppose that $\mathcal{P}$ avoids $\mathcal{P}_{(3,1,1), 5}$ and $\mathcal{P}_{(4,2,1,1), 6}$, i.e. $\mathcal{P}$ is not ladder-climbing. Then the following are satisfied.

(A) For $w \in \mathfrak{S}_{n}$, if $\mathcal{P}-\mathrm{RS}(w)=(P T, Q T)$ then $P T$ is a $\mathcal{P}$-tableau and $Q T$ is a standard Young tableau.

(B) For $w \in \mathfrak{S}_{n}$, if $\mathcal{P}-\operatorname{RS}(w)=(P T, Q T)$ then $\{n-x \mid x \in \operatorname{des} \mathcal{P}(w)\}=\operatorname{des}(Q T)$.

(C) For $w \in \mathfrak{S}_{n}$, If $\mathcal{P}$-RS $(w)=(P T, Q T)$ then $w \sim_{\mathcal{P}} \operatorname{read}(P T)$.

(D) For $w \in \mathfrak{S}_{n}$, if $\mathcal{P}-\mathrm{RS}(w)=(P T, Q T)$ then the length of the first column of $P T$ is equal to

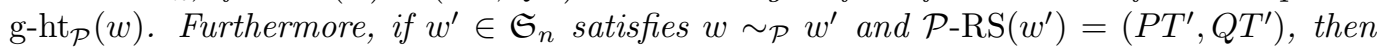
the lengths of the first column of $P T$ and $P T^{\prime}$ are the same.

(E) If $w=\operatorname{read}(P T)$ for some $P T \in \mathcal{P}-\mathrm{Tab}_{\lambda}$ then $\mathcal{P}-\mathrm{RS}(w)=\left(P T, \omega\left(T_{\lambda}\right)\right)$ where $\omega: \mathrm{SYT}_{\lambda} \rightarrow$ $\mathrm{SYT}_{\lambda}$ is Schützenberger's evacuation.

(F) If $\alpha=\left(a_{m}, \ldots, a_{1}\right)$ and $\alpha^{\prime}=\left(a_{m}^{\prime}, \ldots, a_{1}^{\prime}\right)$ are two words of the same length then $\mathcal{P}-\operatorname{RS}(\alpha)=$ $\mathcal{P}-\operatorname{RS}\left(\alpha^{\prime}\right) \Leftrightarrow \alpha=\alpha^{\prime}$.

(G) $\mathcal{P}$-RS restricts to a bijection $\mathcal{P}$-RS : $\mathfrak{S}_{n} \stackrel{\sim}{\rightarrow} \bigsqcup_{\lambda \vdash n} \mathcal{P}-\mathrm{Tab}_{\lambda} \times \mathrm{SYT}_{\lambda}$.

\section{EXAMPLES}

In this section we give various examples of the objects that we introduced so far.

7.1. $\mathcal{P}$-Knuth equivalence graphs. Here we provide some examples of $\mathcal{P}$-Knuth equivalence graphs whose generating functions are not a single Schur function. In Figure 9 14, underlined numbers in each word denote its descents and numbers above edges indicate their colors. Vertices with bold borders are reading words of some $\mathcal{P}$-tableaux and vertices of the same colors are the ones that give the same $\mathcal{P}$-tableau under $\mathcal{P}$-RS. Also, two gray vertices in Figure 12 are the ones that insert to \begin{tabular}{ll|l|l}
\hline & 1 & 2 & 2 \\
\hline 5 & 4 & 4
\end{tabular} , which is not a $\mathcal{P}$-tableau for $\mathcal{P}=\mathcal{P}_{(3,1,1), 5}$.

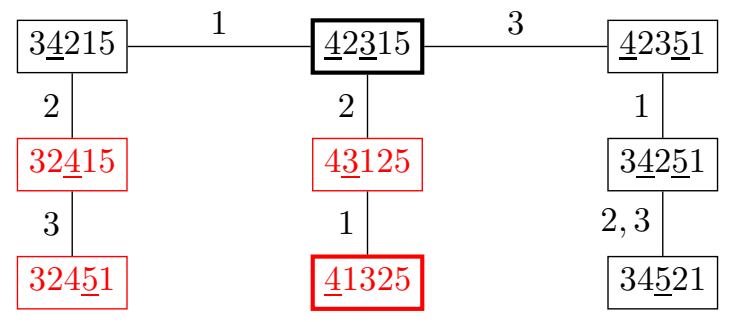

Figure $9 . \mathcal{P}=\mathcal{P}_{(2,2,1), 5}, \gamma_{V}=t^{2}\left(s_{32}+s_{41}\right)$ 


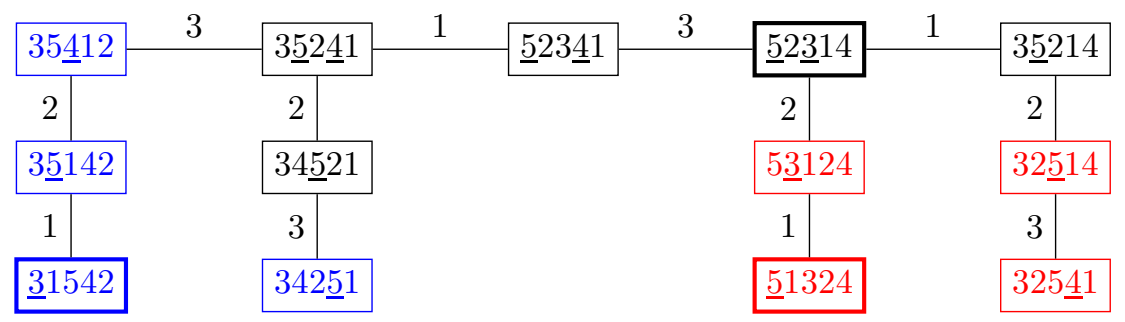

Figure $10 . \quad \mathcal{P}=\mathcal{P}_{(2,1,1), 5}, \gamma_{V}=t^{3}\left(s_{32}+2 s_{41}\right)$

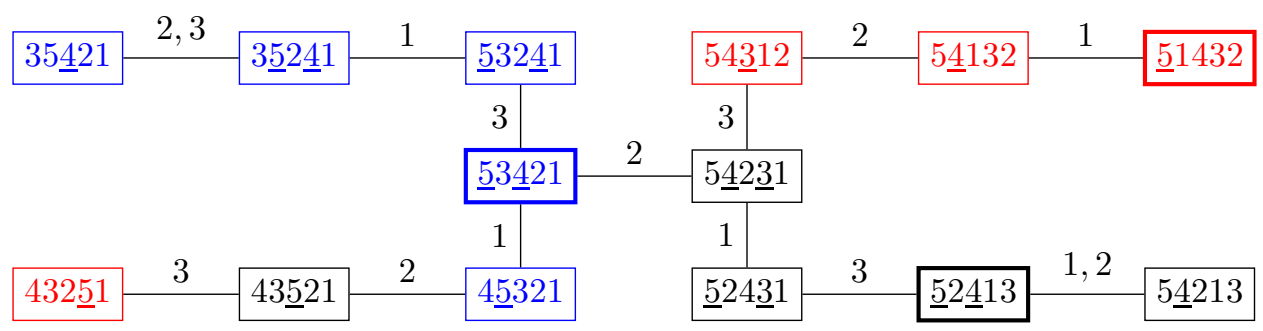

Figure $11 . \mathcal{P}=\mathcal{P}_{(3,2,1), 5}, \gamma_{V}=t^{3}\left(2 s_{32}+s_{41}\right)$

\begin{tabular}{|c|c|c|c|c|c|c|c|c|c|c|}
\hline $4 \underline{5} 213$ & $15 \underline{5} 2 \underline{413}$ & $3 \sqrt{5} 24 \underline{3} 1$ & & & & $\underline{5} 32 \underline{4} 1$ & 1 & $3 \underline{5} 2 \underline{4} 1$ & 3 & $-35 \underline{4} 12$ \\
\hline 2 & 2 & 1 & & & & 3 & & 2 & & 2 \\
\hline $42 \underline{5} 13$ & $5 \underline{4} 123$ & $4 \underline{5} 2 \underline{3} 1$ & 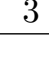 & $-4 \underline{5312}$ & 1 & $-\underline{5} 3 \underline{4} 12$ & & $34 \underline{5} 21$ & & $3 \underline{5} 142$ \\
\hline 3 & 1 & 2 & & & & 2 & & 3 & & 1 \\
\hline $421 \underline{53}$ & $\underline{5} 1423$ & $42 \underline{531}$ & & & & $\underline{53142}$ & & $342 \underline{51}$ & & $\underline{3} 1542$ \\
\hline
\end{tabular}

FIGURE 12. $\mathcal{P}=\mathcal{P}_{(3,1,1), 5}, \gamma_{V}=t^{3}\left(s_{311}+s_{32}+2 s_{41}\right)$ 


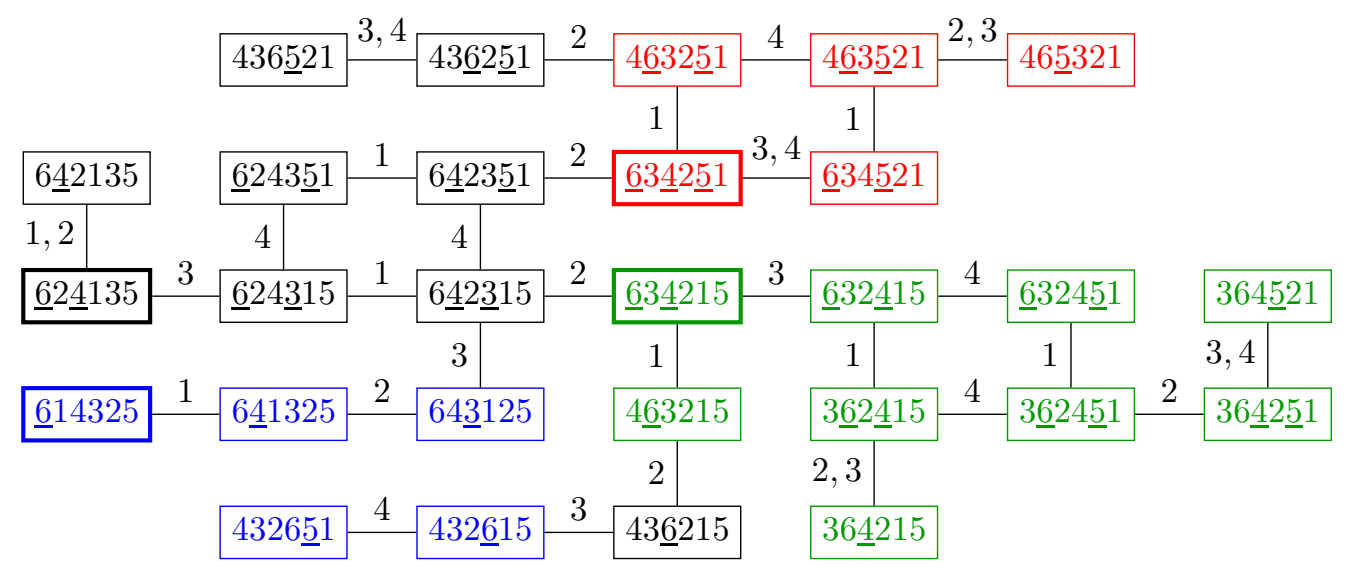

Figure 13. $\mathcal{P}=\mathcal{P}_{(3,3,2,1), 6}, \gamma_{V}=t^{4}\left(s_{33}+2 s_{42}+s_{51}\right)$

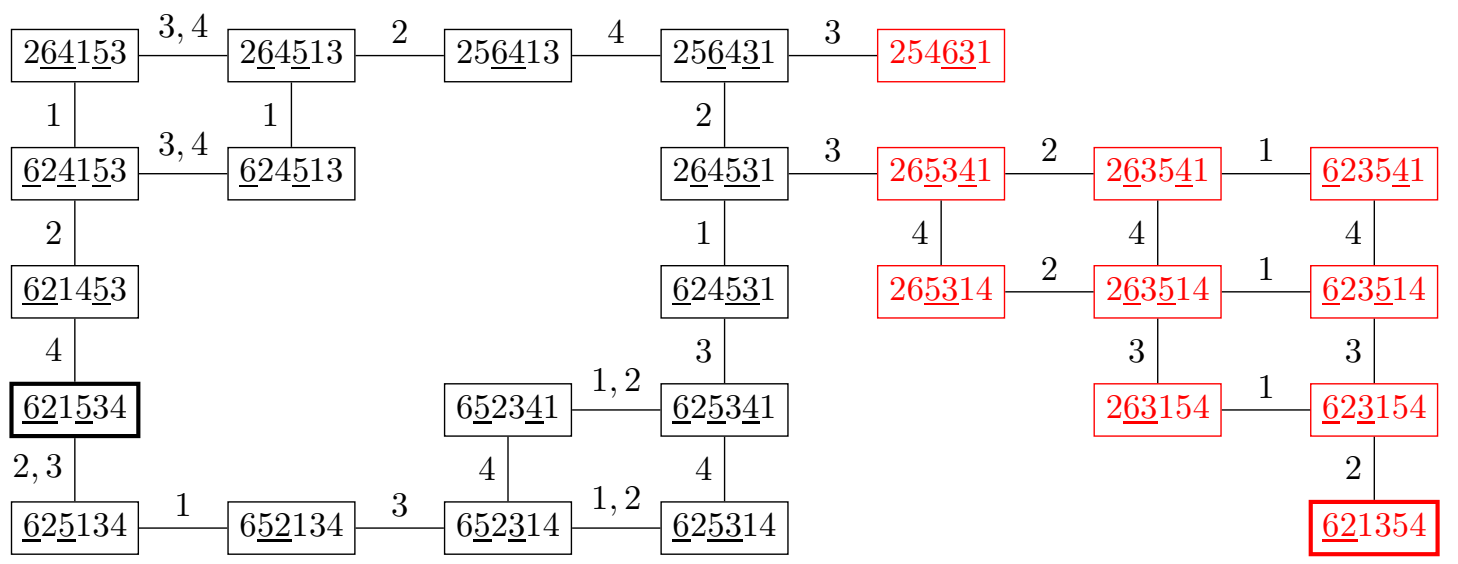

FiguRE 14. $\mathcal{P}=\mathcal{P}_{(4,3,2,1,1), 6}, \gamma_{V}=t^{2}\left(s_{321}+s_{411}\right)$

7.2. Some examples of $\mathbf{A l g}_{\Phi}$ and $\mathbf{A} \lg _{\Psi}$. Here we provide some examples how the algorithms $\mathbf{A l g}_{\Phi}$ and $\mathbf{A l g}_{\Psi}$ work.

Example 7.1 (Figure 15). Suppose that $\mathcal{P}=\mathcal{P}_{(5,3,2,1), 6}, \alpha=(4,3,2)$, and $c=(6,5,1)$. In this case only one step of $\mathbf{A l g}_{\Phi}$ is required to calculate $\Phi(\alpha, c)$, i.e. Case $\operatorname{II}(\mathrm{a})$. Here, the set $A$ in the description of Case II is equal to $\{(0,0),(0,1),(0,2),(1,2)\}$, and thus we have $(h, q)=(1,2)$. It follows that $\Phi(\alpha, c)=((6,5,1),(4,3,2))$.

Example 7.2 (Figure 16). Suppose that $\mathcal{P}=\mathcal{P}_{\mathrm{Stair}(6), 7}, \alpha=(7,5,4,2)$, and $c=(6,3,1)$. Similarly to above, in this case only one step of $\mathbf{A l g}_{\Phi}$ is required to calculate $\Phi(\alpha, c)$, i.e. Case II(b). Here, the set $A$ in the description of Case II is equal to $A=\{(0,0),(1,0),(1,1),(1,2),(2,2),(2,3)\}$, and thus we have $(h, q)=(2,3)$. It follows that $\Phi(\alpha, c)=((7,5,2),(6,4,3,1))$. 

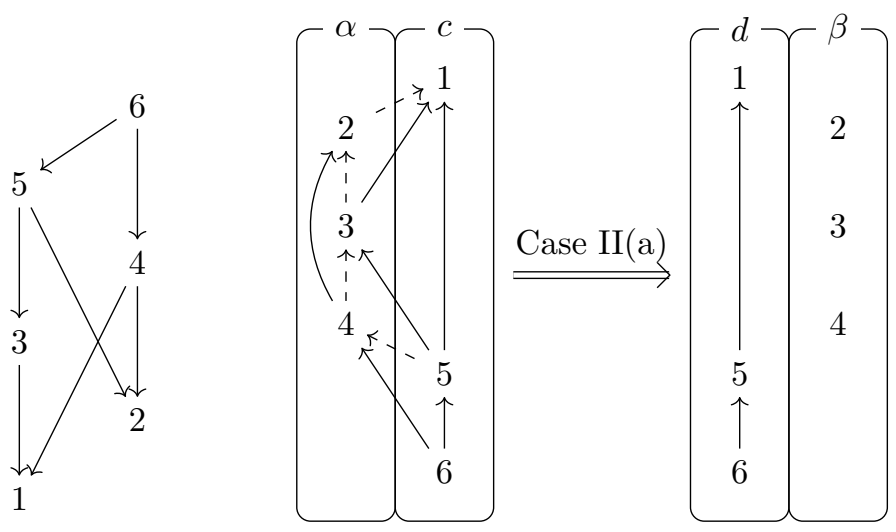

Figure 15. $\operatorname{Alg}_{\Phi}: \mathcal{P}=\mathcal{P}_{(5,3,2,1), 6}, \alpha=(4,3,2)$, and $c=(6,5,1)$
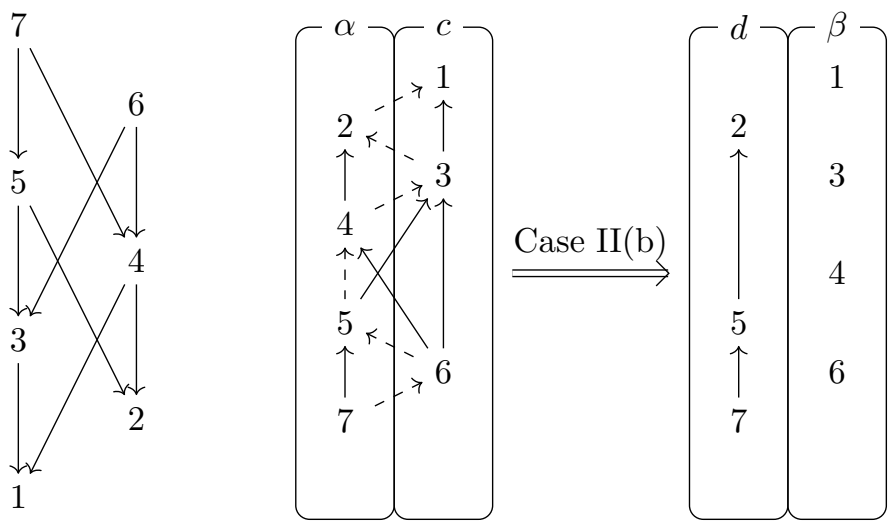

FiguRE 16. $\operatorname{Alg}_{\Phi}: \mathcal{P}=\mathcal{P}_{\text {Stair }(6), 7}, \alpha=(7,5,4,2)$, and $c=(6,3,1)$

Example 7.3 (Figure 17). Suppose that $\mathcal{P}=\mathcal{P}_{(2,1,1), 5}, \alpha=(5,4,2)$ and $c=(3,1)$. We need to process three steps of $\mathbf{A l g}_{\Phi}$ to calculate $\Phi(\alpha, c)$ in this case.

(1) Since $a_{1}=2-\rightarrow_{\mathcal{P}} 1=d_{1}, a_{1}$ is in Case II. Here $A=\{(0,0),(1,0),(0,1),(0,2)\}$, and thus we have $(h, q)=(1,0)$ that is the maximum of $A$ with respect to the lexicographic order even if the choice of $(0,2)$ produces a bigger ladder. We set $b_{1}=2, p=2$ and continue.

(2) Since $a_{2}=4 \rightarrow_{\mathcal{P}} 3=d_{2}, a_{2}$ is in Case II. Here $A=\{(0,0)\}$, and thus $(h, q)=(1,0)$. Note that $\{3,4,5\}$ is not a ladder in $\mathcal{P}$ since $3 \nvdash_{\mathcal{P}} 5$. We set $b_{2}=3, d_{2}=4, p=3$ and continue.

(3) Since $a_{3}=5 \rightarrow \mathcal{P} 4=d_{2}, a_{3}$ is in Case II. Here $A=\{(0,0)\}$, and thus $(h, q)=(1,0)$. We set $b_{3}=4, d_{3}=5$, and terminate the algorithm.

As a result, we have $\Phi((5,4,2),(3,1))=((5,1),(4,3,2))$.

Example 7.4 (Figure 18). Suppose that $\mathcal{P}=\mathcal{P}_{(7,6,5,4,3,2,1), 9}, \alpha=(9,8,7,5,6,3,2,4,1)$ and $c=\emptyset$. We need to process five steps of $\mathbf{A l g}_{\Phi}$ to calculate $\Phi(\alpha, c)$ in this case.

(1) Since the chain is empty, $a_{1}$ is in Case I(a). We set $d_{1}=1, p=2$ and continue. 


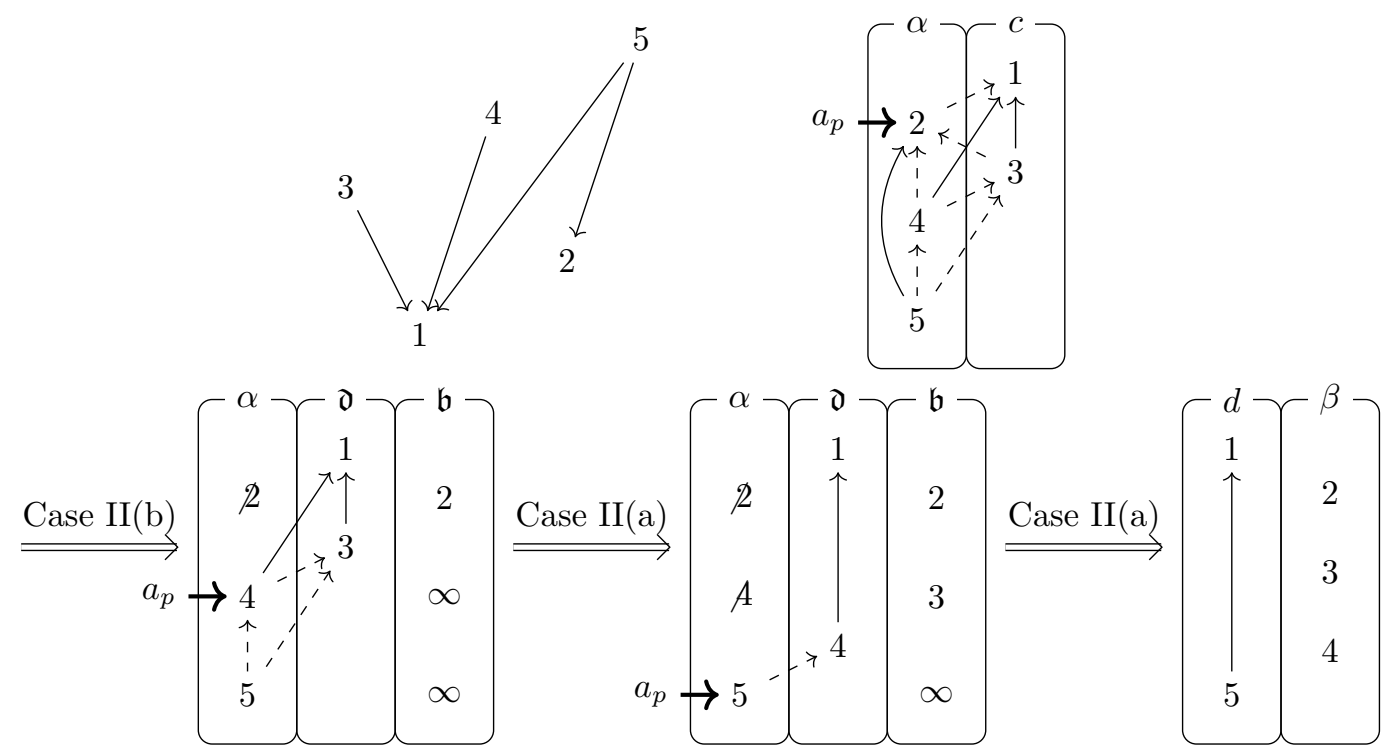

FiguRE 17. $\operatorname{Alg}_{\Phi}: \mathcal{P}=\mathcal{P}_{(2,1,1), 5}, \alpha=(5,4,2)$, and $c=(3,1)$

(2) Since $a_{2}=4>d_{1}=1$ and $4 \rightarrow_{\mathcal{P}} 1, a_{2}$ is in Case $\mathrm{I}(\mathrm{a})$. We set $d_{2}=4, p=3$ and continue.

(3) Since $d_{1}=1<a_{3}=2<d_{2}=4$ and $2 \rightarrow \mathcal{P} 1, a_{3}$ is in Case II. The set $A$ is equal to $\{(0,0),(0,1),(1,1)\}$ thus $(h, q)=(1,1)$, in which case it is in Case II(a). We set $b_{3}=2$, $b_{4}=3, p=5$ and continue.

(4) Since $a_{4}=6>d_{2}=4$ and $6 \rightarrow_{\mathcal{P}} 4, a_{4}$ is in Case $\mathrm{I}(\mathrm{a})$. We set $d_{3}=6, p=6$ and continue.

(5) Since $d_{2}=4<a_{6}=5<d_{3}=6$ and $5 \rightarrow \mathcal{P} 4, a_{6}$ is in Case II. The set $A$ is equal to $\{(0,0),(1,0),(1,1),(1,2),(1,3)\}$ thus $(h, q)=(1,3)$ in which case it is in Case II(b). We set $d_{2}=5, d_{3}=9, b_{6}=4, b_{7}=6, b_{8}=7, b_{9}=8$ and terminate the algorithm.

As a result, we have $\Phi((9,8,7,5,6,3,2,4,1), \emptyset)=((9,5,1),(8,7,6,4, \infty, 3,2, \infty, \infty))$.

Example 7.5 (Figure 19). Suppose that $\mathcal{P}=\mathcal{P}_{(7,6,5,4,3,2,1), 9}, \alpha=(\infty, \infty, 8,7, \infty, 6,4,3,2), c=$ $(9,5,1)$, and $X=\{5,8,9\}$. We need to process five steps of $\operatorname{Alg}_{\Psi}$ to calculate $\Psi_{X}(\alpha, c)$ in this case.

(1) Since $d_{1}=1<a_{1}=2<d_{2}=5$ and $2 \rightarrow \rightarrow_{\mathcal{P}} 1, a_{1}$ is in Case II. The set $A$ is equal to $\{(0,0),(0,1),(0,2),(1,2),(1,3)\}$ thus $(h, q)=(1,3)$ in which case it is in Case II(b). We set $d_{1}=4, d_{2}=6, b_{1}=1, b_{2}=2, b_{3}=3, b_{4}=5, p=5$ and continue.

(2) Since $a_{5}=\infty, 5 \in X$, and $\mathfrak{d} \neq \emptyset, a_{4}$ is in Case $\infty(\mathrm{b})$. We set $\mathfrak{d}=(9,6), b_{5}=4, p=6$ and continue.

(3) Since $d_{1}=6<a_{6}=7<d_{2}=9$ and $7 \rightarrow \mathcal{P} 6, a_{6}$ is in Case II. The set $A$ is equal to $\{(0,0),(0,1),(1,1)\}$ thus $(h, q)=(1,1)$, in which case it is in Case $\mathrm{II}(\mathrm{a})$. We set $b_{6}=7$, $b_{7}=8, p=8$ and continue.

(4) Since $a_{8}=\infty, 8 \in X$, and $\mathfrak{d} \neq \emptyset, a_{8}$ is in Case $\infty(\mathrm{b})$. We set $\mathfrak{d}=(9), b_{8}=6$ and continue.

(5) Since $a_{9}=\infty, 9 \in X$, and $\mathfrak{d} \neq \emptyset, a_{9}$ is in Case $\infty(\mathrm{b})$. We set $\mathfrak{d}=\emptyset, b_{9}=9$ and continue.

As a result, we have $\Psi_{\{5,8,9\}}((\infty, \infty, 8,7, \infty, 6,4,3,2),(9,5,1))=(\emptyset,(9,6,8,7,4,5,3,2,1))$. 

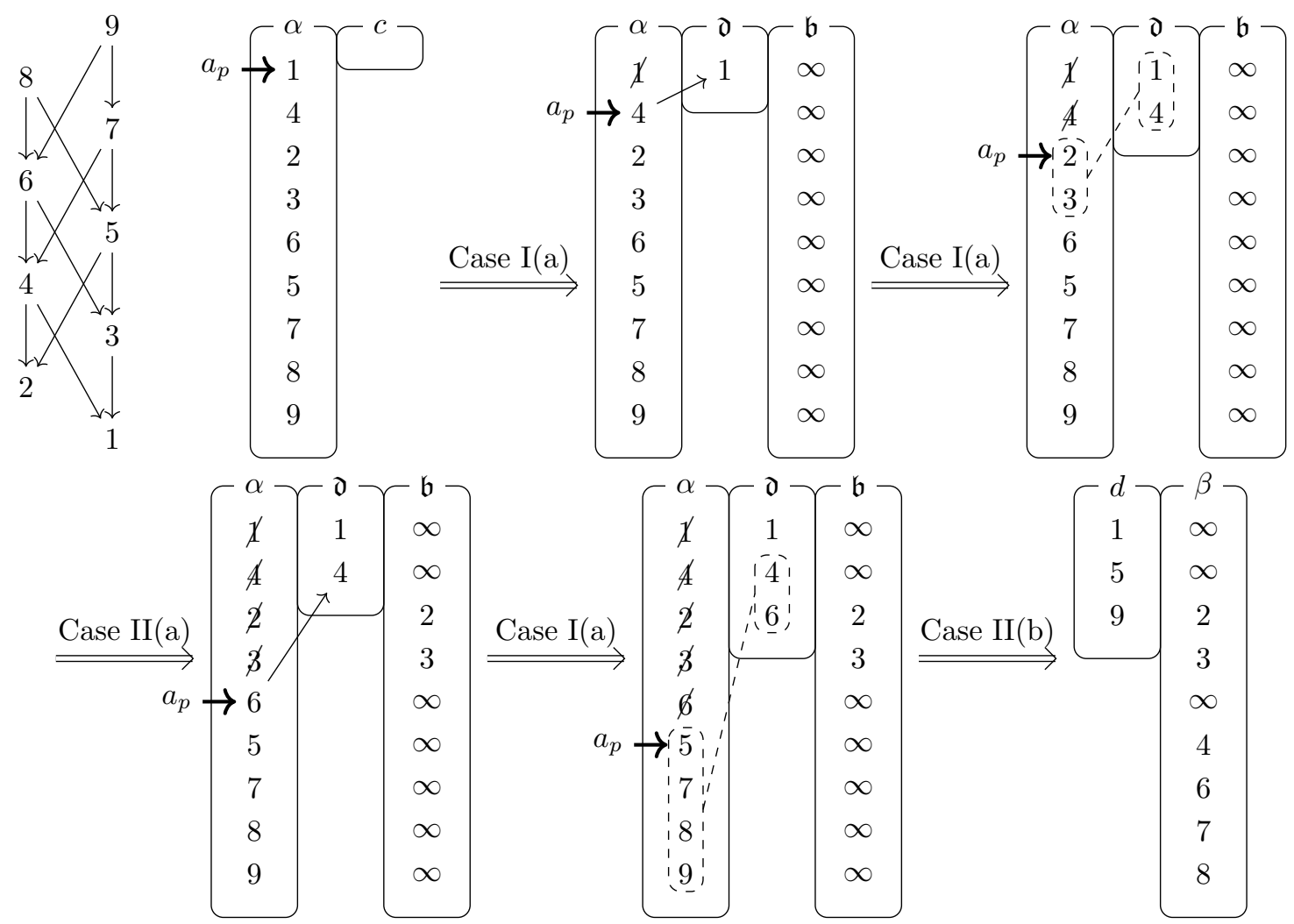

Figure 18. $\operatorname{Alg}_{\Phi}: \mathcal{P}=\mathcal{P}_{(7,6,5,4,3,2,1), 9}, \alpha=(9,8,7,5,6,3,2,4,1)$, and $c=\emptyset$

Remark. Indeed, Example 7.4 and Example 7.5 are "mirror images" to each other. This is not a coincidence but explained in detail in Section 8.6 .

7.3. Some examples of $\mathbf{A l g}_{\mathcal{P} \text {-RS }}$. Here we provide some examples of the algorithm $\mathbf{A l g}_{\mathcal{P} \text {-RS }}$.

Example 7.6. Figure 20 shows the steps of $\mathbf{A l g}_{\mathcal{P} \text {-RS }}$ when $\mathcal{P}=\mathcal{P}_{(2,1), 4}$ and $w$ is an element of $\{3241,3421,4231,4312,4132\}$, the set of vertices of a connected $\mathcal{P}$-Knuth equivalence graph that is

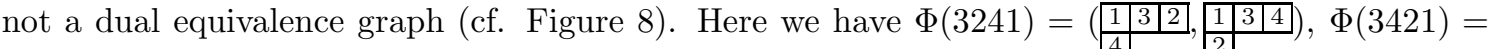

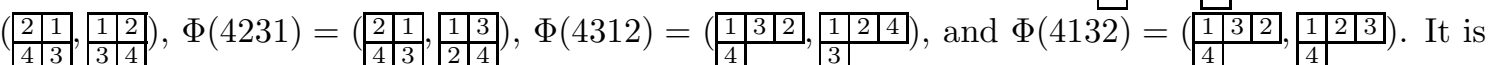
easy to observe in this case that (cf. Theorem 6.1)

(A) the outputs are pairs $(P T, Q T)$ where $P T \in \mathcal{P}-\mathrm{Tab}_{\lambda}, Q T \in \mathrm{SYT}_{\lambda}$ for some $\lambda \vdash 4$,

(B) if $\mathcal{P}-\operatorname{RS}(w)=(P T, Q T)$ then $\operatorname{des}_{\mathcal{P}}(w)=\{4-x \mid x \in \operatorname{des}(Q T)\}$,

(C) $\operatorname{read}\left(\begin{array}{l|l}\hline & 1 \\ \hline\end{array}\right)=4231$ and $\operatorname{read}\left(\begin{array}{ll|l}\hline & 3 & 2\end{array}\right)=4132$ are the vertices of the given connected $\mathcal{P}$-Knuth equivalence graph,

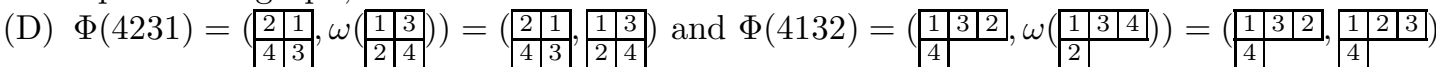
where $\omega$ is Schützenberger's evacuation, and 

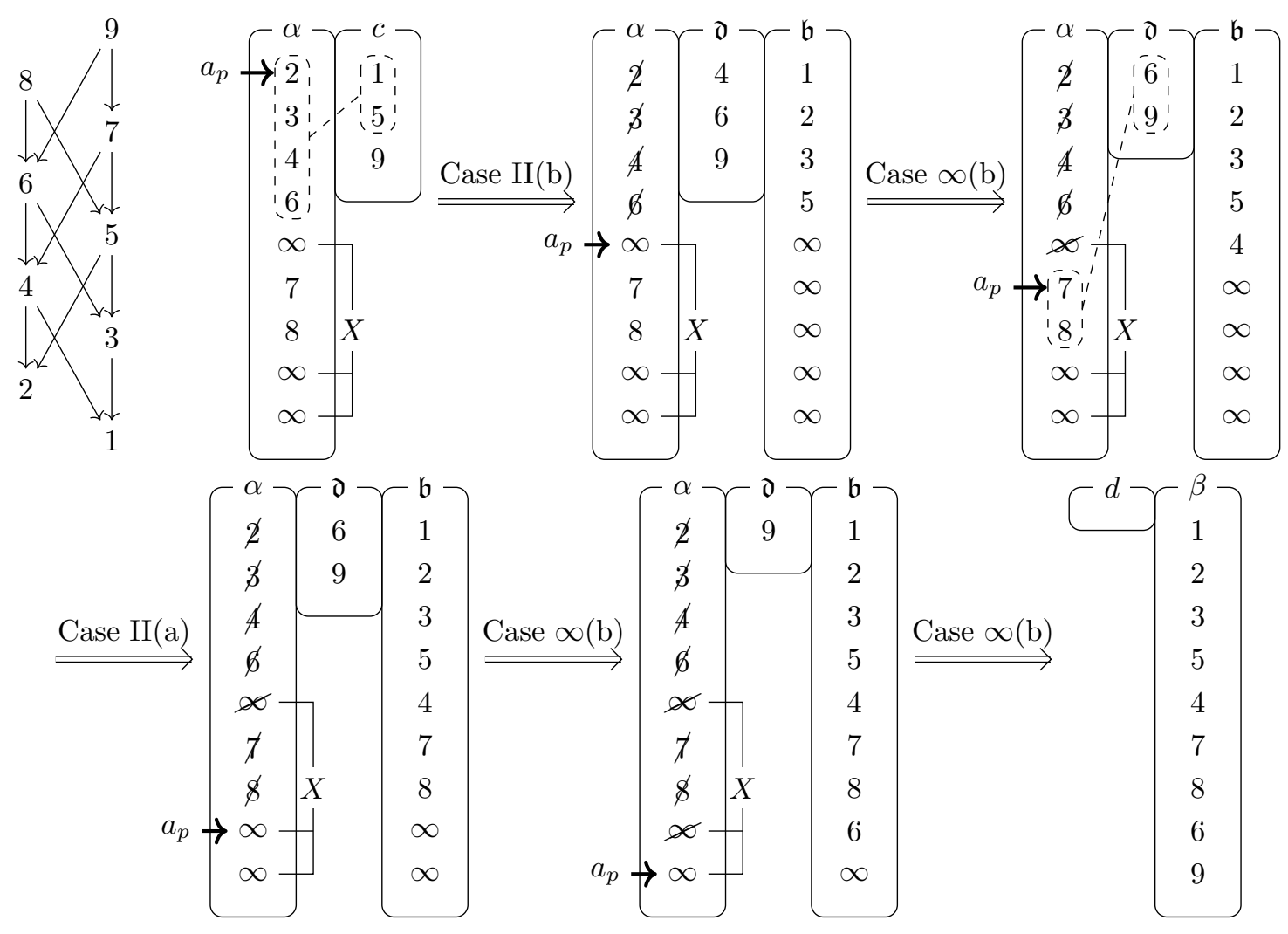

Figure 19. $\operatorname{Alg}_{\Psi}: \mathcal{P}=\mathcal{P}_{(7,6,5,4,3,2,1), 9}, \alpha=(\infty, \infty, 8,7, \infty, 6,4,3,2)$,

$$
c=(9,5,1) \text {, and } X=\{5,8,9\}
$$

(E), (F) $\Phi$ gives a bijection between the given set of vertices and $\left\{\begin{array}{l|l}\frac{2}{4} & \frac{1}{3}\end{array}\right\} \times \mathrm{SYT}_{(2,2)} \sqcup\left\{\begin{array}{l|l|l|}\frac{1}{4} & 3 \mid 2\end{array}\right\} \times$ $\operatorname{SYT}_{(3,1)}$.

Furthermore, its generating function is $t^{2}\left(s_{31}+s_{22}\right)$ as expected by Theorem 4.13 ,

Example 7.7. Figure21]shows the steps of $\mathbf{A l g}_{\mathcal{P} \text {-RS }}$ when $\mathcal{P}=\mathcal{P}_{(7,6,5,4,3,2,1), 9}$ and $w=(9,8,7,5,6,3,2,4,1)$.

Here we have $\Phi(w)=\left(\begin{array}{|l|l|l|l|l|l|l|l|}\hline & 4 & 3 & 2 & 1 & 3 & 4 & 6 \\ \hline 5 & 8 & 7 & 6 \\ \hline & 2 & 7 & 8 & 9\end{array}\right)$. Note that $\left\{9-x \mid x \in \operatorname{des}_{\mathcal{P}}(w)\right\}=\{1,4,6\}=$ $\operatorname{des}\left(\begin{array}{|l|l|l|l|l}\hline & 3 & 4 & 6 \\ \hline 2 & 7 & 8 & 9\end{array}\right)$

5

Example 7.8. Figure 22 shows the steps of $\mathbf{A l g}_{\mathcal{P} \text {-RS }}$ when $\mathcal{P}=\mathcal{P}_{(9,8,6,6,4,3,2,2,1), 10}$ and $w=$

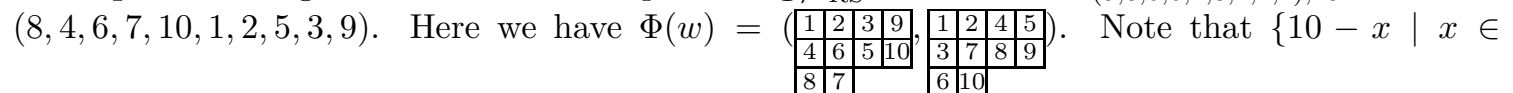
$\left.\operatorname{des}_{\mathcal{P}}(w)\right\}=\{2,5,9\}=\operatorname{des}\left(\begin{array}{|l|l|l|l}\hline 1 & 2 & 4 & 5 \\ \hline 3 & 7 & 8 & 9 \\ \hline 6 & 10 & \end{array}\right.$. 

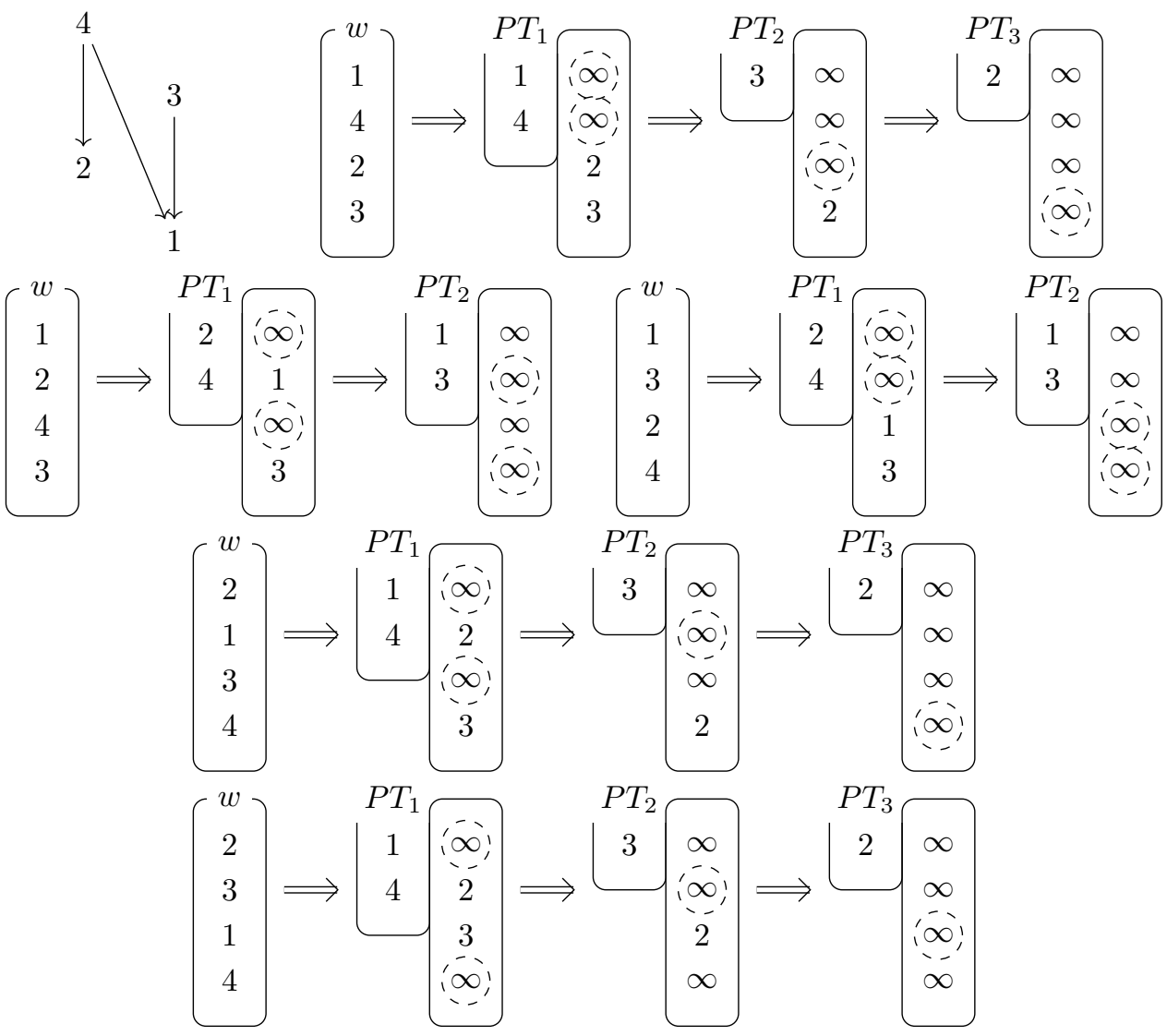

FIGURE 20. $\operatorname{Alg}_{\mathcal{P}-\mathrm{RS}}: \mathcal{P}=\mathcal{P}_{(2,1), 4}, w \in\{3241,3421,4231,4312,4132\}$

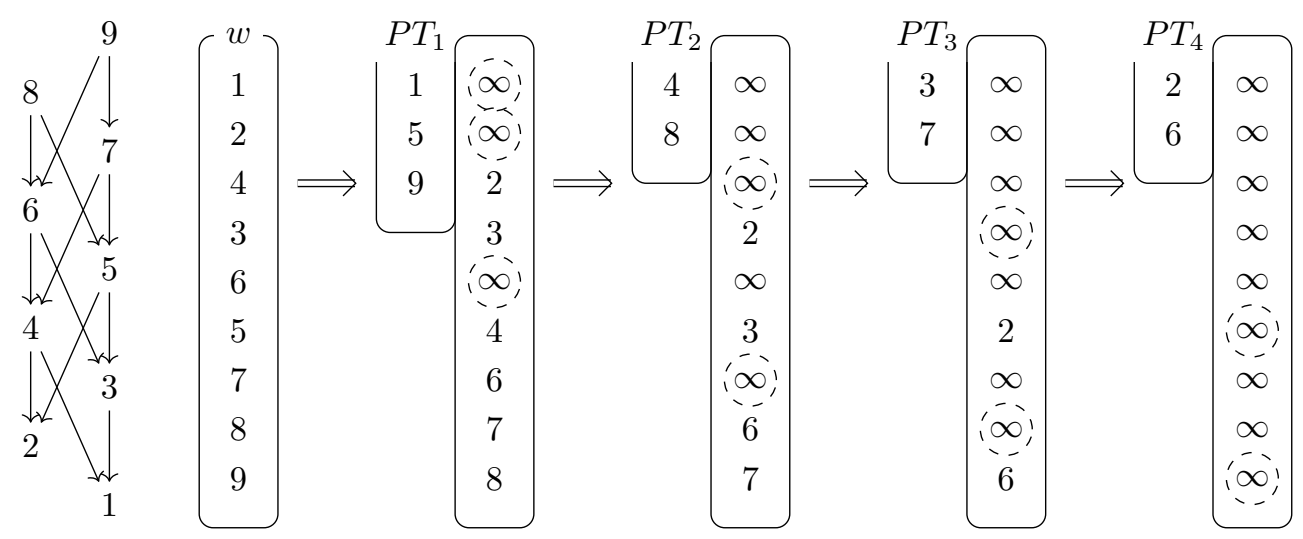

FIGURE 21. $\operatorname{Alg}_{\mathcal{P}-\mathrm{RS}}: \mathcal{P}=\mathcal{P}_{(7,6,5,4,3,2,1), 9}, w=(9,8,7,5,6,3,2,4,1)$ 


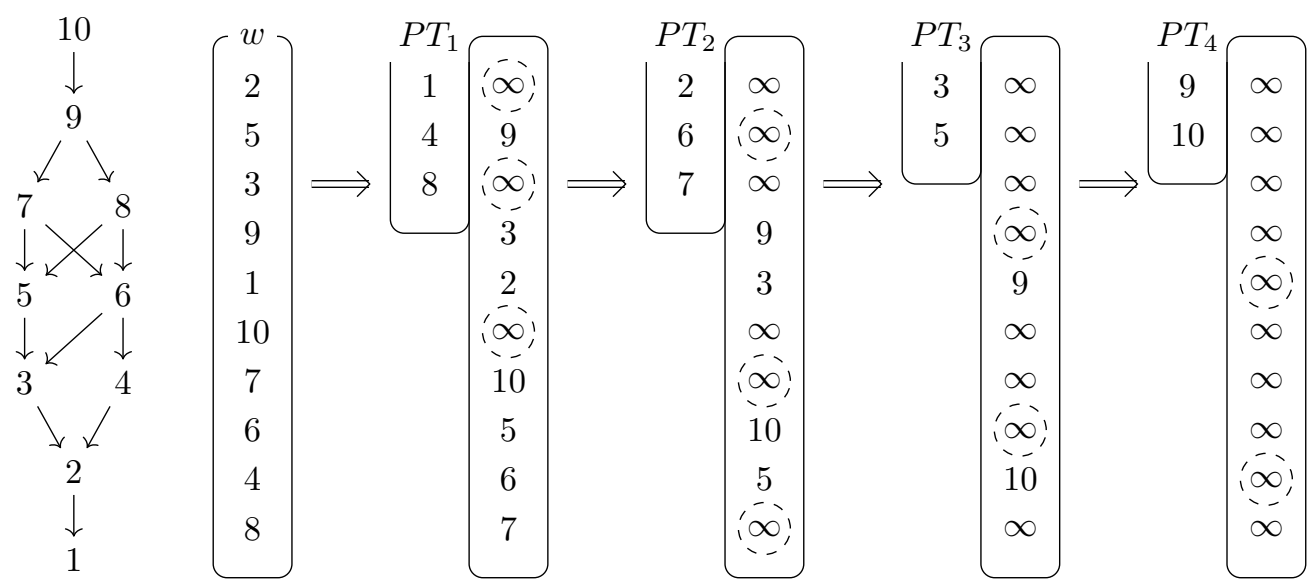

FigURE 22. $\operatorname{Alg}_{\mathcal{P}-\mathrm{RS}}: \mathcal{P}=\mathcal{P}_{(9,8,6,6,4,3,2,2,1), 10}, w=(8,4,6,7,10,1,2,5,3,9)$

7.4. Some pathologies for ladder-climbing partial orders. Here we provide some examples when $\mathcal{P}$-RS does not produce a desired output when $\mathcal{P}$ is ladder-climbing.

Example 7.9. Figure 23 shows the steps of $\mathbf{A l g}_{\mathcal{P} \text {-RS }}$ when $\mathcal{P}=\mathcal{P}_{(3,1,1), 5}$ and $w=(3,4,5,2,1)$. Here we see that $\Phi(w)=\left(\begin{array}{l|l|l|l|l|l}3 & 1 & 2\end{array}, \begin{array}{lll}1 & 2 & 5\end{array}\right)$. However, $P T$ in this case is not a $\mathcal{P}$-tableau since $3 \rightarrow \mathcal{P} 1$.

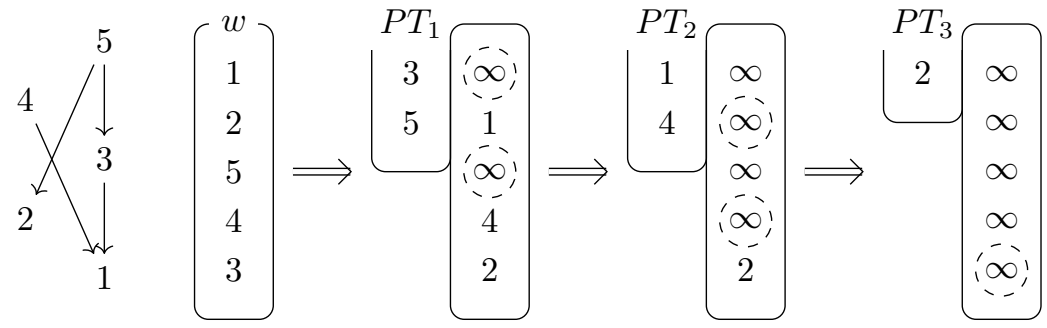

FIGURE 23. $\operatorname{Alg}_{\mathcal{P} \text {-RS }}: \mathcal{P}=\mathcal{P}_{(3,1,1), 5}, w=(3,4,5,2,1)$

Example 7.10. Figure 24] shows the steps of $\mathbf{A l g}_{\mathcal{P} \text {-RS }}$ when $\mathcal{P}=\mathcal{P}_{(4,2,1,1), 6}$ and $w=(4,3,6,5,2,1)$.

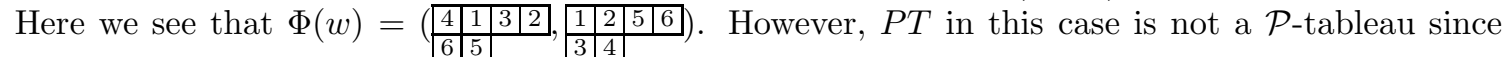
$4 \rightarrow \mathcal{P} 1$.

Example 7.11. Figure 25 shows the steps of $\mathbf{A l g}_{\mathcal{P} \text {-RS }}$ when $\mathcal{P}=\mathcal{P}_{(5,3,2,1,1), 7}$ and $w=(3,1,5,6,7,4,2)$.

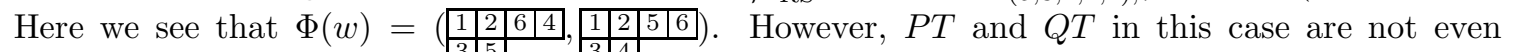
tableaux. 

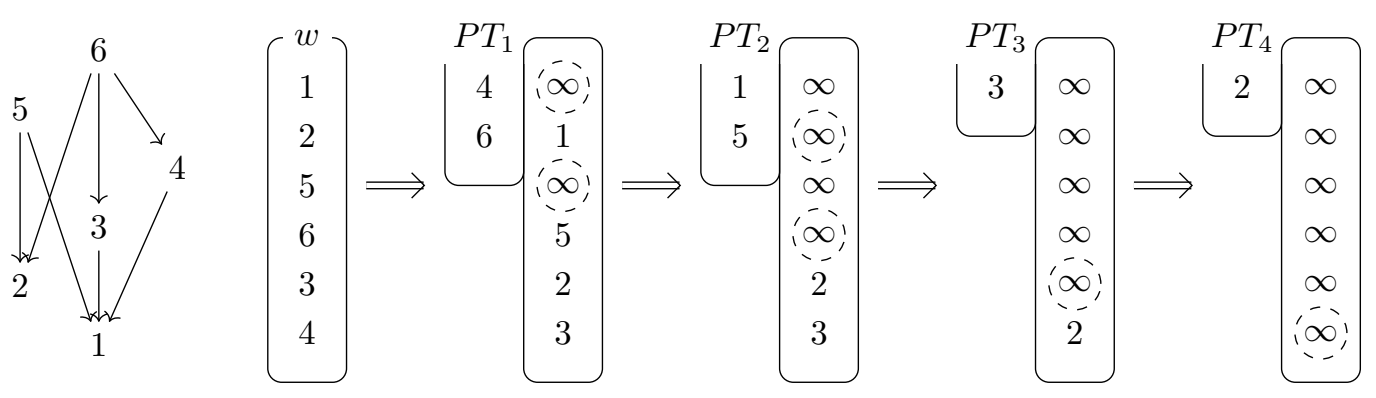

FIGURE 24. $\operatorname{Alg}_{\mathcal{P}-\mathrm{RS}}: \mathcal{P}=\mathcal{P}_{(4,2,1,1), 6}, w=(4,3,6,5,2,1)$
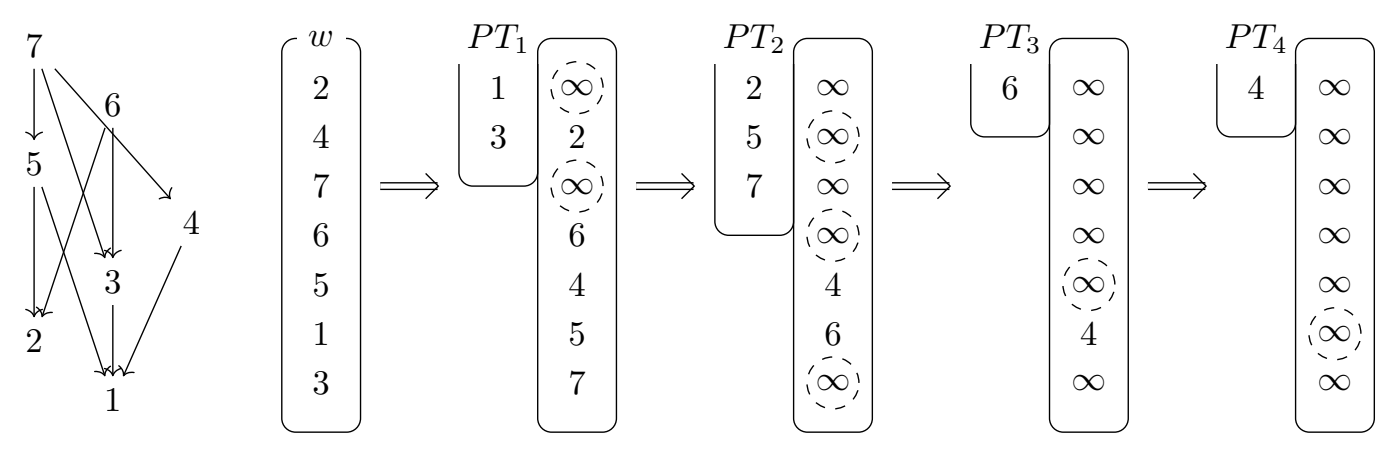

FIGURE 25. $\operatorname{Alg}_{\mathcal{P}-\mathrm{RS}}: \mathcal{P}=\mathcal{P}_{(5,3,2,1,1), 7}, w=(3,1,5,6,7,4,2)$

\section{Proof of Proposition 4.10, 5.5, and 5.6}

8.1. Proof of Proposition 4.10, We start with the following lemma.

Lemma 8.1. Let $\mathcal{P}$ be a natural unit interval order on $[1, n]$. Suppose that we are given $a, b \in[1, n]$ and $w \in \mathfrak{S}_{n}$ that satisfy $w^{-1}(a)<w^{-1}(b)$ and $a \rightarrow_{\mathcal{P}} b$. Then the following are equivalent:

(1) $(a, b) \notin \mathrm{g}^{-h} t_{\mathcal{P}}(w)$.

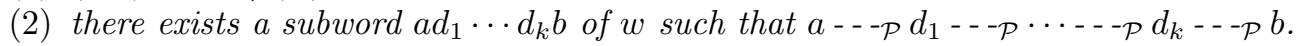

(3) there exists a subword $a d_{1} \cdots d_{k}$ b of $w$ such that $a-\rightarrow_{\mathcal{P}} d_{1}-\rightarrow_{\mathcal{P}} \cdots \rightarrow \rightarrow_{\mathcal{P}} d_{k} \rightarrow \rightarrow_{\mathcal{P}} b$.

(4) there exists a subword $a d_{1} \cdots d_{k} b$ of $w$ such that $a \rightarrow \rightarrow_{\mathcal{P}} d_{1} \rightarrow \rightarrow_{\mathcal{P}} \cdots \rightarrow \mathcal{P} d_{k} \rightarrow \rightarrow_{\mathcal{P}} b$ and $\left\{a, d_{1}, \ldots, d_{k}, b\right\}$ is a ladder in $\mathcal{P}$.

Proof. (1) $\Leftrightarrow(2)$ by definition, and $(4) \Rightarrow(3) \Rightarrow(2)$ is clear. It remains to show $(2) \Rightarrow(4)$. To this end, suppose that $a d_{1} \cdots d_{k} b$ is a subword of $w$ such that $a--_{\mathcal{P}} d_{1}--_{\mathcal{P}} \cdots \cdots-\mathcal{p} d_{k}--_{\mathcal{p}} b$ and we construct another subword satisfying the condition of (4).

For simplicity we set $d_{0}=a$ and $d_{k+1}=b$. First, let $d_{i}$ be the last element in the subword $d_{0} d_{1} \cdots d_{k} d_{k+1}$ such that $d_{0}--_{\mathcal{p}} d_{i}$. By removing $d_{1}, d_{2}, \ldots, d_{i-1}$ if necessary, without loss of generality we may assume that it is $d_{1}$. Similarly we let $d_{j}$ be the last element in the subword $d_{0} d_{1} \cdots d_{k} d_{k+1}$ such that $d_{1}--\mathcal{p} d_{j}$. By removing $d_{2}, \ldots, d_{j-1}$ if necessary, without loss of generality we may assume that it is $d_{2}$. We iterate this process, and thus we may assume that $d_{i+1}$ is the last element in $d_{0} d_{1} \cdots d_{k} d_{k+1}$ such that $d_{i-\cdots} d_{i+1}$ for $i \in[0, k]$. 
We claim that $d_{i} \rightarrow \rightarrow \mathcal{P} d_{i+1}$ for any $i \in[0, k]$. For the sake of contradiction suppose otherwise, i.e. $d_{i}{ }_{--\mathcal{P}} d_{i+1}$ and let $i \in[0, k]$ be the smallest element such that $d_{i}{ }^{--\mathcal{P}} d_{i+1}$. If $i>0$, then $d_{i-1} \rightarrow \mathcal{P} d_{i}$ by assumption and thus $d_{i-1}-\mathcal{P}_{\mathcal{P}} d_{i+1}$ by Condition $(\pitchfork)$, which contradicts the fact that $d_{i}$ is the last element satisfying $d_{i-1}-_{-\mathcal{P}} d_{i}$. If $i=0$, i.e. $a \leftarrow_{--\mathcal{P}} d_{1}$, then we claim that $a \leftarrow_{\mathcal{P}} d_{j}$ for any $j \geq 2$, which in particular contradicts the fact that $a \rightarrow_{\mathcal{P}} b=d_{k+1}$. Thus suppose otherwise. Then there exists $j$ such that $a \rightarrow_{\mathcal{P}} d_{j}$, and we choose such $j$ to be the minimum one. Then we have $a<d_{j-1}, d_{j-1}--_{\mathcal{P}} d_{j}$, and $a \rightarrow_{\mathcal{P}} d_{j}$, which contradicts Condition ( $\pitchfork$ ). (Note that this argument is valid even when $j=2$.)

Now for any $i \in[1, k]$, we have $d_{i-1} \rightarrow_{\mathcal{P}} d_{i} \rightarrow_{\mathcal{P}} d_{i+1}$, and thus $d_{i-1} \rightarrow_{\mathcal{P}} d_{i+1}$ since again $d_{i}$ is the last element satisfying $d_{i-1-\mathcal{-}^{-}} d_{i}$. But this means that $\left\{d_{0}, d_{1}, \ldots, d_{k}, d_{k+1}\right\}$ is a ladder in $\mathcal{P}$, which is what we want to prove.

Let us start proving Proposition 4.10, Let $w=\cdots x y z \cdots$ and $w^{\prime}=\cdots x^{\prime} y^{\prime} z^{\prime} \cdots$ such that the $\mathcal{P}$-Knuth move connecting $w$ and $w^{\prime}$ shuffles $\{x, y, z\}=\left\{x^{\prime}, y^{\prime}, z^{\prime}\right\}$. Suppose that $\mathcal{I}:=$ $\left(p_{1}, \ldots, p_{s}, r_{1}, \ldots, r_{u}, q_{1}, \ldots, q_{t}\right)$ is the longest subword of genuine $\mathcal{P}$-inversions in $w$ such that $\underline{\mathcal{I}} \cap\{x, y, z\}=\left\{r_{1}, \ldots, r_{u}\right\}$. In particular we have g-ht $_{\mathcal{P}}(w)=s+t+u$. Note that $s, t \geq 0$ and $u \in[0,2]$. ( $u$ cannot be 3 since otherwise $x \rightarrow_{\mathcal{P}} y \rightarrow_{\mathcal{P}} z$ in which case there is no $\mathcal{P}$-Knuth move shuffling $x, y, z$.)

We may assume $s, t \geq 2$. Indeed, we add $n+1, n+2$ and $0,-1$ to the poset $([1, n], \mathcal{P})$ so that $n+2 \rightarrow_{\mathcal{P}} n+1 \rightarrow_{\mathcal{P}} i \rightarrow_{\mathcal{P}} 0 \rightarrow_{\mathcal{P}}-1$ for any $i \in[1, n]$. Then we replace $w$ and $w^{\prime}$ with $(n+2, n+1)+w+(0,-1)$ and $(n+2, n+1)+w^{\prime}+(0,-1)$, respectively. After this procedure, $\mathcal{P}$ still remains to avoid $\mathcal{P}_{(3,1,1), 5}$ and $\mathcal{P}_{(4,2,1,1), 6}$. Also, any longest subword of genuine $\mathcal{P}$-inversions in $w$ and $w^{\prime}$ contains $n+2, n+1,0$, and -1 , which in particular increases $s$ and $t$ by 2 , respectively. (Here, we have $p_{1}=n+2, p_{2}=n+1, q_{t-1}=0$, and $q_{t}=-1$.)

We will construct the subword $\mathcal{I}^{\prime}$ of genuine $\mathcal{P}$-inversions of length $s+t+u$ on $w^{\prime}$, which contains $p_{1}, \ldots, p_{s-1}, q_{2}, \ldots, q_{t}$. It means in particular that g-ht $\mathcal{P}_{\mathcal{P}}\left(w^{\prime}\right) \geq s+t+u$, which proves the claim by symmetry. Note that we possibly change only $p_{s}, r_{1}, \ldots, r_{u}, q_{1}$ part from $\mathcal{I}$ to obtain $\mathcal{I}^{\prime}$. Therefore, the entries before $p_{s-1}$ and after $q_{2}$ in $w$ and $w^{\prime}$ do not affect this process. By removing such entries if necessary, it suffices to assume that $s=t=2$, i.e. we have $\mathcal{I}=\left(p_{1}, p_{2}, r_{1}, \ldots, r_{u}, q_{1}, q_{2}\right)$.

Let $a, b, c \in[1, n]$ be such that $a<b<c$ and $\{a, b, c\}=\{x, y, z\}=\left\{x^{\prime}, y^{\prime}, z^{\prime}\right\}$. From now on we argue case-by-case based on $\left.\mathcal{P}\right|_{\{a, b, c\}}$ and $u \in[0,2]$.

8.1.1. $\left.\mathcal{P}\right|_{\{a, b, c\}} \simeq \mathcal{P}_{\emptyset, 3}$. There is no $\mathcal{P}$-Knuth move in this case, so there is nothing to prove.

8.1.2. $\left.\mathcal{P}\right|_{\{a, b, c\}} \simeq \mathcal{P}_{(1), 3}$. We have $a \leftarrow^{-\mathcal{P}} b \leftarrow^{-\mathcal{P}} c$ and $a \leftarrow_{\mathcal{P}} c$. There is only one $\mathcal{P}$-Knuth move in this case: $[\cdots b c a \cdots] \stackrel{\mathcal{P}}{m}[\cdots c a b \cdots]$.

- $u=0$ case. In this case we have $\mathcal{I}=p_{1} p_{2} q_{1} q_{2}$ and in particular $\left(p_{2}, q_{1}\right) \in \mathrm{g}-\operatorname{inv}_{\mathcal{P}}(w)$. If $\left(p_{2}, q_{1}\right) \in \operatorname{g-inv}_{\mathcal{P}}\left(w^{\prime}\right)$ then we are done since we may set $\mathcal{I}^{\prime}=\mathcal{I}$. From now on we assume $\left(p_{2}, q_{1}\right) \notin$ $\operatorname{g-inv}_{\mathcal{P}}\left(w^{\prime}\right)$.

First consider the case when $w=[\cdots b c a \cdots]$ and $w^{\prime}=[\cdots c a b \cdots]$. By Lemma 8.1, there exists a subword $\mathcal{J}:=p_{2} d_{1} \cdots d_{k} q_{1}(k \geq 1)$ in $w^{\prime}$ where $p_{2} \rightarrow \mathcal{P} d_{1} \rightarrow \mathcal{P} \cdots \rightarrow \mathcal{P} d_{k} \rightarrow \mathcal{P} q_{1}$ and $\underline{\mathcal{J}}$ is a ladder in $\mathcal{P}$. As $\left(p_{2}, q_{1}\right) \in$ g-inv $_{\mathcal{P}}(w)$, it means that the order of some elements in $\mathcal{J}$ should be switched under the $\mathcal{P}$-Knuth move so that it no longer prohibits $\left(p_{2}, q_{1}\right)$ from being a genuine 
$\mathcal{P}$-inversion of $w$. Then the only possibility is that $\mathcal{J}=p_{2} d_{1} \cdots d_{v-1} c b d_{v+2} \cdots d_{k} q_{1}$, i.e. $d_{v}=c$ and $d_{v+1}=b$ for some $v \in[1, k-1]$.

We claim that we may choose $\mathcal{I}^{\prime}:=p_{1} p_{2} a q_{2}$. To this end we need to check that $\left(p_{2}, a\right),\left(a, q_{2}\right) \in$ g-ht $_{\mathcal{P}}\left(w^{\prime}\right)$. First if $\left(p_{2}, a\right) \notin \mathrm{g}^{-h} \mathrm{It}_{\mathcal{P}}\left(w^{\prime}\right)$ then as $p_{2}>c \rightarrow \mathcal{P}$ a there exists a subword $p_{2} e_{1} \cdots e_{l} a$

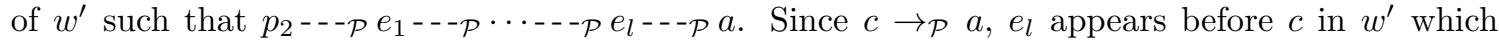
means that $p_{2} e_{1} \cdots e_{l} a$ is also a subword of $w$. However, this contradicts $\left(p_{2}, q_{1}\right) \in \mathrm{g}^{-h} t_{\mathcal{P}}(w)$ as

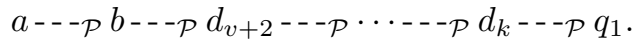

This time suppose that $\left(a, q_{2}\right) \notin{\mathrm{g}-h t_{\mathcal{P}}}\left(w^{\prime}\right)$. Since $b>q_{1} \rightarrow_{\mathcal{P}} q_{2}$ and $b-\rightarrow_{\mathcal{P}} a$, by Condition (内) we should have $a>q_{2}$. First suppose that $a \rightarrow \rightarrow_{\mathcal{P}} q_{2}$. If $v<k-1$ then $b \rightarrow_{\mathcal{P}} q_{1} \rightarrow_{\mathcal{P}} q_{2}$ and $b \rightarrow \mathcal{P} a \rightarrow_{\mathcal{P}} q_{2}$, which contradicts Lemma 3.4. Thus $v=k-1$, i.e. $\mathcal{J}=\cdots c b q_{1}$. However, direct calculation shows that $\mathcal{P}$ restricted to $\left\{c, b, q_{1}, a, q_{2}\right\}$ is isomorphic to $\mathcal{P}_{(3,1,1), 5}$, which is again a contradiction.

Thus we have $a \rightarrow_{\mathcal{P}} q_{2}$, and by Lemma 8.1 there exists a subword $a e_{1} \cdots e_{l} q_{2}$ of $w^{\prime}(l \geq 1)$ such that $a \rightarrow \mathcal{P} e_{1} \rightarrow \mathcal{P} \cdots \rightarrow \mathcal{P} e_{l} \rightarrow \mathcal{P} q_{2}$ and $\left\{a, e_{1}, \ldots, e_{l}, q_{2}\right\}$ is a ladder in $\mathcal{P}$. By Lemma 3.11] it follows that $\left\{c, b, a, e_{1}, \ldots, e_{l}, q_{2}\right\}$ is also a ladder in $\mathcal{P}$. By assumption we have $c \rightarrow_{\mathcal{P}} q_{1} \rightarrow_{\mathcal{P}} q_{2}$, and also $q_{1} \notin\left\{c, b, a, e_{1}, \ldots, e_{l}, q_{2}\right\}$; it is clear that $q_{1} \neq c, b, a, q_{2}$, and if $q_{1}=e_{i}$ for some $i$ then it means that $\left(q_{1}, q_{2}\right) \notin \mathrm{g}$-htt $(w)$. In other words, $q_{1}$ is climbing the ladder $\left\{c, b, a, e_{1}, \ldots, e_{l}, q_{2}\right\}$, which contradicts the assumption on $\mathcal{P}$.

It remains to consider the case when $w=[\cdots c a b \cdots]$ and $w^{\prime}=[\cdots b c a \cdots]$. By arguing similarly, we should be able to find a subword $\mathcal{J}=p_{2} d_{1} \cdots d_{v-1} b a d_{v+2} \cdots d_{k} q_{1}$ of $w^{\prime}$ with the same properties as above. Then we may choose $\mathcal{I}^{\prime}=p_{1} c q_{1} q_{2}$, which can also be shown analogously. We omit the details.

- $u=1$ case. We have $\mathcal{I}=p_{1} p_{2} r_{1} q_{1} q_{2}$ where $r_{1} \in\{a, b, c\}$. First consider the case when $w=[\cdots b c a \cdots]$ and $w^{\prime}=[\cdots c a b \cdots]$. Using Lemma 8.1, one can easily show that if $r_{1}=a$ then we may set $\mathcal{I}^{\prime}=\mathcal{I}$ and we are done.

Now suppose that $r_{1}=b$. We claim that we may choose $\mathcal{I}^{\prime}=p_{1} p_{2} a q_{1} q_{2}$. To this end we need to verify that $\left(p_{2}, a\right),\left(a, q_{1}\right) \in \mathrm{g}^{-h t_{\mathcal{P}}}\left(w^{\prime}\right)$. First if $\left(p_{2}, a\right) \notin \mathrm{g}-\mathrm{ht}_{\mathcal{P}}\left(w^{\prime}\right)$ then as $p_{2} \rightarrow_{\mathcal{P}} b>a$ there exists a subword $p_{2} e_{1} \cdots e_{l} a$ of $w^{\prime}$ such that $p_{2} \rightarrow \rightarrow_{\mathcal{P}} e_{1} \rightarrow \rightarrow_{\mathcal{P}} \cdots \rightarrow \rightarrow_{\mathcal{P}} e_{l} \rightarrow \mathcal{P} a$. Since $b \rightarrow \mathcal{P} a$, either $e_{l}=b$ or $e_{l--_{\mathcal{P}}} b$ by Condition $(\pitchfork)$, which means that $\left(p_{2}, b\right) \notin \mathrm{g}-\mathrm{ht}_{\mathcal{P}}(w)$. This is a contradiction and thus we have $\left(p_{2}, a\right) \in \mathrm{g}^{-h t_{\mathcal{P}}}\left(w^{\prime}\right)$. On the other hand, since $b \rightarrow_{\mathcal{P}} q_{1}$ and $b-\rightarrow_{\mathcal{P}} a$ we have $a>q_{1}$ by Condition $(\pitchfork)$. Now if $\left(a, q_{1}\right) \notin \mathrm{g}-\mathrm{ht}_{\mathcal{P}}\left(w^{\prime}\right)$, i.e. there exists a subword $a e_{1} \cdots e_{l} q_{1}(l \geq 0)$ of $w^{\prime}$ such that $a \rightarrow \rightarrow_{\mathcal{P}} e_{1} \rightarrow \rightarrow_{\mathcal{P}} \cdots \rightarrow \rightarrow_{\mathcal{P}} e_{l} \rightarrow \rightarrow_{\mathcal{P}} q_{1}$ (note that $e_{1}$ cannot be $b$ ), then since $b \rightarrow \rightarrow_{\mathcal{P}} a$ it follows that $\left(b, q_{1}\right) \notin \mathrm{g}^{-h t_{\mathcal{P}}}(w)$. This is a contradiction, and thus we have $\left(a, q_{1}\right) \in \mathrm{g}^{-\mathrm{ht}_{\mathcal{P}}}\left(w^{\prime}\right)$ as desired.

If $r_{1}=c$, then by the same argument one can easily show that we may choose $\mathcal{I}^{\prime}=p_{1} p_{2} b q_{1} q_{2}$. We omit the details.

It remains to consider the case when $w=[\cdots c a b \cdots]$ and $w^{\prime}=[\cdots b c a \cdots]$. Similarly to above, we may set $\mathcal{I}^{\prime}=p_{1} p_{2} c q_{1} q_{2}$ if $r_{1} \in\{b, c\}$ and $\mathcal{I}^{\prime}=p_{1} q_{2} b q_{1} q_{2}$ if $r_{1}=a$. Again we omit the details.

- $u=2$ case. We have $\mathcal{I}=p_{1} p_{2} c a q_{1} q_{2}$. Then using Lemma 8.1 one can easily show that $\mathcal{I}^{\prime}=\mathcal{I}$ satisfies the desired properties. 
8.1.3. $\left.\mathcal{P}\right|_{\{a, b, c\}} \simeq \mathcal{P}_{(1,1), 3}$. We have $a \leftarrow \mathcal{P} b \leftarrow_{-\mathcal{P}} c$ and $a \leftarrow \mathcal{P} c$. Here we have two kinds of $\mathcal{P}$-Knuth moves: $[\cdots b c a \cdots] \stackrel{\mathcal{P}}{m}[\cdots b a c \cdots]$ and $[\cdots c b a \cdots] \stackrel{\mathcal{P}}{m}[\cdots c a b \cdots]$.

- $u=0$ case. In this case we have $\mathcal{I}=p_{1} p_{2} q_{1} q_{2}$ and in particular $\left(p_{2}, q_{1}\right) \in \operatorname{g-inv}_{\mathcal{P}}(w)$. Since the relative position of $b$ and $c$ does not change by the $\mathcal{P}$-Knuth moves in this case, one can easily show that $\left(p_{2}, q_{1}\right) \in \mathrm{g}$-inv $\mathcal{P}_{\mathcal{P}}\left(w^{\prime}\right)$ by using Lemma 8.1. Then we are done since we may set $\mathcal{I}^{\prime}=\mathcal{I}$.

- $u=1$ case. We have $\mathcal{I}=p_{1} p_{2} r_{1} q_{1} q_{2}$ where $r_{1} \in\{a, b, c\}$. Again, since the relative position of $b$ and $c$ does not change by the $\mathcal{P}$-Knuth moves in this case, one can easily show that we may set $\mathcal{I}^{\prime}=\mathcal{I}$ by using Lemma 8.1 .

- $u=2$ case. We have $\mathcal{I}=p_{1} p_{2} r_{1} r_{2} q_{1} q_{2}$ where either $\left(r_{1}, r_{2}\right)=(b, a)$ or $\left(r_{1}, r_{2}\right)=(c, a)$. Since the relative position of $b$ and $c$ does not change by the $\mathcal{P}$-Knuth moves in this case, we only need to consider the situations when the relative position of $r_{1}$ and $r_{2}$ changes under the moves. We have two cases to consider.

The first case is when $\mathcal{I}=p_{1} p_{2} b a q_{1} q_{2}, w=[\cdots c b a \cdots]$, and $w^{\prime}=[\cdots c a b \cdots]$. We claim that we may choose $\mathcal{I}^{\prime}=p_{1} p_{2} c a q_{1} q_{2}$. To this end we need to check $\left(p_{2}, c\right) \in \mathrm{g}^{-h t_{\mathcal{P}}}\left(w^{\prime}\right)$. (It is trivial that $(c, a) \in{\mathrm{g}-h t_{\mathcal{P}}}\left(w^{\prime}\right)$ in this case.) Since $p_{2} \rightarrow_{\mathcal{P}} b$ and $c \rightarrow_{\mathcal{P}} b$, we have $p_{2}>c$ by Condition $(\pitchfork)$. Thus if $\left(p_{2}, c\right) \notin \mathrm{g}-\mathrm{ht}_{\mathcal{P}}\left(w^{\prime}\right)$ then there exists a subword $p_{2} e_{1} \cdots e_{l} c(l \geq 0)$ of $w^{\prime}$ such that $p_{2} \rightarrow{ }_{\mathcal{P}} e_{1} \rightarrow \rightarrow_{\mathcal{P}} \cdots \rightarrow \rightarrow_{\mathcal{P}} e_{l} \rightarrow \rightarrow \mathcal{P} c$. However, since $c \rightarrow \mathcal{P} b$, it implies $\left(p_{2}, b\right) \notin \mathrm{g}^{-h h_{\mathcal{P}}}(w)$, which is a contradiction.

The remaining case is when $\mathcal{I}=p_{1} p_{2} c a q_{1} q_{2}, w=[\cdots b c a \cdots]$, and $w^{\prime}=[\cdots b a c \cdots]$. Here one can show that we may choose $\mathcal{I}^{\prime}=p_{1} p_{2} b a q_{1} q_{2}$ similarly to above. We omit the details.

8.1.4. $\left.\mathcal{P}\right|_{\{a, b, c\}} \simeq \mathcal{P}_{(2), 3}$. This case is completely analogous to the above case when $\left.\mathcal{P}\right|_{\{a, b, c\}} \simeq$ $\mathcal{P}_{(1,1), 3}$ if one "reverses" the words therein. We again omit the details.

8.1.5. $\left.\mathcal{P}\right|_{\{a, b, c\}} \simeq \mathcal{P}_{(2,1), 3}$. We have $a \leftarrow \mathcal{P} b \leftarrow \mathcal{P} c$. Here we have two kinds of $\mathcal{P}$-Knuth moves: $[\cdots b c a \cdots] \stackrel{\mathcal{P}}{\stackrel{m}{m}[\cdots b a c \cdots]}$ and $[\cdots a c b \cdots] \stackrel{\mathcal{P}}{\stackrel{m}{m}[\cdots c a b \cdots]}$.

- $u=0$ case. In this case we have $\mathcal{I}=p_{1} p_{2} q_{1} q_{2}$. It is easy to show that $\left(p_{2}, q_{1}\right) \in \mathrm{g}^{-h t_{\mathcal{P}}}(w)$ implies $\left(p_{2}, q_{1}\right) \in{\mathrm{g}-h t_{\mathcal{P}}}_{(}\left(w^{\prime}\right)$, and thus we may set $\mathcal{I}^{\prime}=\mathcal{I}$.

- $u=1$ case. We have $\mathcal{I}=p_{1} p_{2} r_{1} q_{1} q_{2}$ where $r_{1} \in\{a, b, c\}$. Again, one can easily show that we may set $\mathcal{I}^{\prime}=\mathcal{I}$.

- $u=2$ case. We have $\mathcal{I}=p_{1} p_{2} r_{1} r_{2} q_{1} q_{2}$ where $\left(r_{1}, r_{2}\right) \in\{(b, a),(c, a),(c, b)\}$. Here we only need to consider the situations when the relative position of $r_{1}$ and $r_{2}$ changes under the $\mathcal{P}$-Knuth moves. We have two cases to consider.

The first case is when $\mathcal{I}=p_{1} p_{2} c a q_{1} q_{2}, w=[\cdots b c a \cdots]$, and $w^{\prime}=[\cdots b a c \cdots]$. Here we may choose $\mathcal{I}^{\prime}=p_{1} p_{2} b a q_{1} q_{2}$. Trivially $(b, a) \in \mathrm{g}^{-h}{ }_{\mathcal{P}}\left(w^{\prime}\right)$, so it suffices to check that $\left(p_{2}, b\right) \in \mathrm{g}^{-h} t_{\mathcal{P}}\left(w^{\prime}\right)$. If we suppose otherwise then since $p_{2} \rightarrow_{\mathcal{P}} c \rightarrow_{\mathcal{P}} b$ there exists a subword $p_{2} e_{1} \cdots e_{l} b(l>0)$ in $w^{\prime}$ such that $p_{2} \rightarrow \rightarrow_{\mathcal{P}} e_{1} \rightarrow \rightarrow_{\mathcal{P}} \cdots \rightarrow_{\mathcal{P}} e_{l} \rightarrow \rightarrow_{\mathcal{P}} b$ and $\left\{p_{2}, e_{1}, \ldots, e_{l}, b\right\}$ is a ladder in $\mathcal{P}$. However, $c \notin\left\{p_{2}, e_{1}, \ldots, e_{l}, b\right\}$ and $p_{2} \rightarrow_{\mathcal{P}} c \rightarrow_{\mathcal{P}} b$ which means that $c$ is climbing the aforementioned ladder, which is a contradiction. Thus we have $\left(p_{2}, b\right) \in \mathrm{g}^{-h t_{\mathcal{P}}}\left(w^{\prime}\right)$.

The remaining case is when $\mathcal{I}=p_{1} p_{2} c a q_{1} q_{2}, w=[\cdots c a b \cdots]$, and $w^{\prime}=[\cdots a c b \cdots]$. Here we may choose $\mathcal{I}^{\prime}=p_{1} p_{2} c b q_{1} q_{2}$ similarly to above. We omit the details. 
We exhausted all the cases and thus conclude the statement.

8.2. Proof of Proposition 5.5](A), Here, we write $(\cdots \underline{a b c} \cdots) \stackrel{\mathcal{P}}{m}\left(\cdots a^{\prime} b^{\prime} c^{\prime} \cdots\right)$ for $\left\{a^{\prime}, b^{\prime}, c^{\prime}\right\}=$ $\{a, b, c\}$ to indicate the location of a $\mathcal{P}$-Knuth move (that is the underlined part). We start with the following lemma. After this, we use wavy underlines, e.g. $\cdots x_{1} \cdots x_{k} \cdots \sim_{\mathcal{P}} \cdots y_{1} \cdots y_{k} \cdots$ for $\left\{x_{1}, \ldots, x_{k}\right\}=\left\{y_{1}, \ldots, y_{k}\right\}$ to indicate the part where Lemma 8.2 is applied.

Lemma 8.2. Suppose that a fixed natural unit interval order $\mathcal{P}$ is given.

(1) If $b_{1} \leftarrow_{\mathcal{P}} b_{2} \leftarrow_{\mathcal{P}} \cdots \leftarrow_{\mathcal{P}} b_{k}$ and $a<b_{1}$ then $a b_{k} \cdots b_{1} \sim_{\mathcal{P}} b_{k} \cdots b_{2} a b_{1}$.

(2) If $b_{1} \leftarrow_{\mathcal{P}} b_{2} \leftarrow_{\mathcal{P}} \cdots \leftarrow_{\mathcal{P}} b_{k}$ and $a>b_{k}$ then $b_{k} \cdots b_{1} a \sim_{\mathcal{P}} b_{k} a b_{k-1} \cdots b_{1}$.

(3) If $b_{1} \leftarrow-\mathcal{P} b_{2} \leftarrow--\mathcal{P} \cdots \leftarrow-\mathcal{P} b_{k}, x \leftarrow \mathcal{P} y$, and $y<b_{1}$ then $y x b_{k} \cdots b_{1} \sim_{\mathcal{P}} y b_{k} \cdots b_{1} x$.

(4) If $b_{1}{ }^{\leftarrow-\mathcal{P}} b_{2} \leftarrow-\mathcal{P}^{\prime} \cdots \leftarrow-\mathcal{P}^{-} b_{k}, x \leftarrow \mathcal{P} y$, and $x>b_{k}$ then $b_{k} \cdots b_{1} y x \sim_{\mathcal{P}} y b_{k} \cdots b_{1} x$.

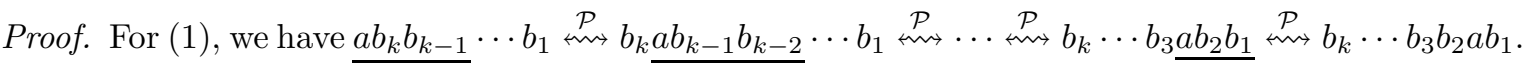

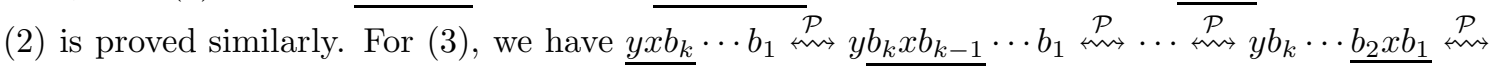
$y b_{k} \cdots b_{2} b_{1} x$. (4) is proved similarly.

We prove (A) by showing that each step in the column insertion algorithm respects the $\mathcal{P}$-Knuth equivalence. It is sufficient to consider when the input is given by $\left(\alpha=\left(a_{m}, \ldots, a_{1}\right), c=\left(c_{l}, \ldots, c_{1}\right)\right)$ such that $c_{1} \leftarrow \mathcal{P} c_{2} \leftarrow \mathcal{P} \cdots \leftarrow \mathcal{P} c_{l}, \alpha=\alpha^{f}$, and only one step of $\mathbf{A l g}_{\Phi}$ is required to obtain the output. We argue case-by-case.

Case I(a). There is nothing to prove since $\alpha+c=d$ and $\beta=(\infty)$.

Case I(b). The length of $\alpha$ equals 1, i.e. $\alpha=(a)$ for some $a$. First suppose that $c_{i}<a<c_{i+1}$ and

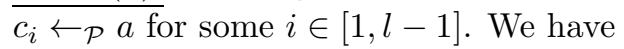

$$
\begin{aligned}
& a c_{l} \cdots c_{i+2} c_{i+1} c_{i} \cdots c_{1} \sim \mathcal{P} c_{l} \cdots c_{i+2} \underline{a c_{i+1}} c_{i} c_{i-1} \cdots c_{1} \\
& \stackrel{\mathcal{P}}{m} c_{l} \cdots c_{i+2} a c_{i} c_{i+1} c_{i-1} \cdots c_{1} \sim \mathcal{P} c_{l} \cdots c_{i+2} a c_{i} \cdots c_{1} c_{i+1} .
\end{aligned}
$$

which proves the claim. It remains to show that $a c_{l} \cdots c_{1} \sim_{\mathcal{P}} c_{l} \cdots c_{2} a c_{1}$ if $c_{1} \leftarrow \mathcal{P} c_{2} \leftarrow \mathcal{P} \cdots \leftarrow \mathcal{P} c_{l}$ and $a<c_{1}$, but it follows directly from Lemma 8.2 .

Case II(a). It suffices to prove the following lemma.

Lemma 8.3. Suppose that $c_{1} \leftarrow \mathcal{P} \cdots \leftarrow \mathcal{P} c_{l}, c_{i}<a_{1}<a_{2}<\cdots<a_{m}<c_{i+1}$ for some $i \in[1, l-1]$, and $\left\{c_{i}, a_{1}, \ldots, a_{m}, c_{i+1}\right\}$ is a ladder in $\mathcal{P}$. Then $a_{m} \cdots a_{1} c_{l} \cdots c_{1} \sim_{\mathcal{P}} c_{l} \cdots c_{1} a_{m} \cdots a_{1}$.

Proof. Since we have

$$
\begin{aligned}
& a_{m} \cdots a_{1} c_{l} c_{l-1} c_{l-2} \cdots c_{1} \sim_{\mathcal{P}} c_{l} a_{m} \cdots a_{1} c_{l-1} c_{l-2} c_{l-3} \cdots c_{1} \sim_{\mathcal{P}} \cdots \\
& \sim_{\mathcal{P}} c_{l} \cdots c_{i+3} a_{m} \cdots a_{1} c_{i+2} c_{i+1} c_{i} \cdots c_{1} \sim_{\mathcal{P}} c_{l} \cdots c_{i+2} a_{m} \cdots a_{1} c_{i+1} c_{i} \cdots c_{1} \quad \text { and } \\
& c_{l} \cdots c_{i+1} c_{i} a_{m} \cdots a_{1} c_{i-1} c_{i-2} \cdots c_{1} \sim_{\mathcal{P}} c_{l} \cdots c_{i} c_{i-1} a_{m} \cdots a_{1} c_{i-2} c_{i-3} \cdots c_{1} \sim_{\mathcal{P}} \cdots \\
& \sim_{\mathcal{P}} c_{l} \cdots c_{3} c_{2} a_{m} \cdots a_{1} c_{1} \sim \mathcal{P} c_{l} \cdots c_{3} c_{2} c_{1} a_{m} \cdots a_{1}
\end{aligned}
$$


it is enough to consider the case when $i=1$ and $l=2$. However, in this case we have

$$
\begin{aligned}
& a_{m} \cdots a_{2} \underline{a_{1} c_{2} c_{1}} \stackrel{\mathcal{P}}{\leftrightarrow} a_{m} \cdots a_{3} \underline{a_{2} c_{2} a_{1}} c_{1} \stackrel{\mathcal{P}}{\stackrel{P}{P} \cdots \stackrel{\mathcal{P}}{a_{m}}} \underline{a_{m} c_{2} a_{m-1}} a_{m-2} \cdots a_{1} c_{1}
\end{aligned}
$$

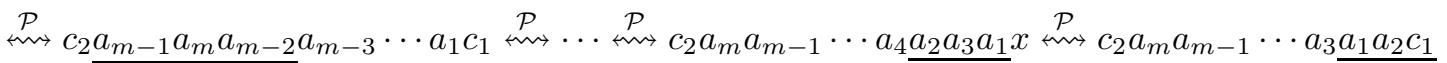

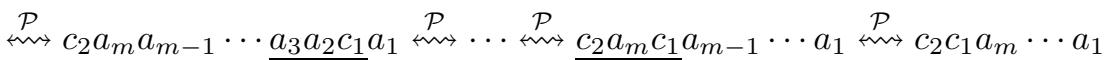

as desired.

Case II(b). Suppose that there exist $s, t$ and $0=u(0)<u(1)<\cdots<u(s)=m$ such that $\overline{c_{t+i}<a_{u(i-1)+1}}<\cdots<a_{u(i)}$ for $i \in[1, s]$. Also we assume that $\left\{c_{t+1}, \ldots, c_{t+s}, a_{1}, \ldots, a_{m}\right\}$ is a ladder in $\mathcal{P}$. Note that if $t+s<l$ then we may assume that $c_{t+s+1} \rightarrow \mathcal{P} a_{i}$ for any $i \in[1, m]$ by maximality in Case II.

We argue by induction on $s \geq 1$. Assume that $s=1$, i.e. $c_{t+1}<a_{1}<\cdots<a_{m}$ and $c_{t+2} \rightarrow_{\mathcal{P}} a_{i}$ for any $i \in[1, m]$ if $t+1<l$. First if $m=1$, then we have

$$
\begin{aligned}
& \overbrace{a_{1} c_{l} \cdots c_{t+2}} c_{t+1} \cdots c_{1} \sim_{\mathcal{P}} c_{l} \cdots c_{t+3} \underline{a_{1} c_{t+2} c_{t+1}} c_{t} \cdots c_{1} \\
& \stackrel{\mathcal{P}}{m} c_{l} \cdots c_{t+2} \underline{a_{1} c_{t+1} c_{t}} c_{t-1} \cdots c_{1} \\
& \stackrel{\mathcal{P}}{\leftrightarrow} c_{l} \cdots c_{t+2} a_{1} c_{t} c_{t+1} c_{t-1} c_{t-2} \cdots c_{1} \stackrel{\mathcal{P}}{\stackrel{P}{P}} \cdot \\
& \stackrel{\mathcal{P}}{\stackrel{P}{m} c_{l} \cdots c_{t+2} a_{1} c_{t} \cdots c_{2} c_{t+1} c_{1}} \\
& \stackrel{\mathcal{P}}{m} c_{l} \cdots c_{t+2} a_{1} c_{t} \cdots c_{2} c_{1} c_{t+1}
\end{aligned}
$$

which proves the claim. In general, we have

$$
\begin{aligned}
a_{m} \cdots a_{1} c_{l} \cdots c_{1} & \sim_{\mathcal{P}} a_{m} \cdots a_{2} c_{l} \cdots c_{t+2} a_{1} c_{t} \cdots c_{1} c_{t+1} \\
& \sim_{\mathcal{P}} a_{m} \cdots a_{3} c_{l} \cdots c_{t+2} a_{2} c_{t} \cdots c_{1} a_{1} c_{t+1} \sim_{\mathcal{P}} \cdots \\
& \sim_{\mathcal{P}} c_{l} \cdots c_{t+2} a_{m} c_{t} \cdots c_{1} a_{m-1} \cdots a_{1} c_{t+1}
\end{aligned}
$$

by iterating the above process $m$ times.

Now suppose that the induction step is valid up to $s-1$. Then we have

$$
\begin{aligned}
& a_{u(s)} \cdots a_{1} c_{l} \cdots c_{1} \\
& \quad \sim_{\mathcal{P}} a_{u(s)} \cdots a_{u(1)+1} c_{l} \cdots c_{1} a_{u(1)} \cdots a_{1} \\
& \sim_{\mathcal{P}} c_{l} \cdots c_{t+s+1} a_{u(s)} a_{u(s-1)} \cdots a_{u(2)} c_{t+1} \cdots c_{1}+a_{u(s)-1} \cdots a_{u(s-1)+1} c_{t+s} \\
& \quad+a_{u(s-1)-1} \cdots a_{u(s-2)+1} c_{t+s-1}+\cdots+a_{u(2)-1} \cdots a_{u(1)+1} c_{t+2} a_{u(1)} \cdots a_{1} \\
& \quad \sim_{\mathcal{P}} a_{u(s)-1} \cdots a_{u(s-1)+1} c_{t+s}+a_{u(s-1)-1} \cdots a_{u(s-2)+1} c_{t+s-1}+\cdots \\
& \quad+a_{u(2)-1} \cdots a_{u(1)+1} c_{t+2} a_{u(1)} \cdots a_{1}+c_{l} \cdots c_{t+s+1} a_{u(s)} a_{u(s-1)} \cdots a_{u(2)} c_{t+1} \cdots c_{1} \\
& \quad \sim_{\mathcal{P}} a_{u(s)-1} \cdots a_{u(s-1)+1} c_{t+s}+a_{u(s-1)-1} \cdots a_{u(s-2)+1} c_{t+s-1}+\cdots+a_{u(2)-1} \cdots a_{u(1)+1} c_{t+2} \\
& \quad+c_{l} \cdots c_{t+s+1} a_{u(s)} a_{u(s-1)} \cdots a_{u(2)} a_{u(1)} c_{t} \cdots c_{1}+a_{u(1)} \cdots a_{1} c_{t+1} \\
& \quad \sim_{\mathcal{P}} c_{l} \cdots c_{t+s+1} a_{u(s)} a_{u(s-1)} \cdots a_{u(2)} a_{u(1)} c_{t} \cdots c_{1}+a_{u(s)-1} \cdots a_{u(s-1)+1} c_{t+s} \\
& \quad+a_{u(s-1)-1} \cdots a_{u(s-2)+1} c_{t+s-1}+\cdots+a_{u(2)-1} \cdots a_{u(1)+1} c_{t+2}+a_{u(1)} \cdots a_{1} c_{t+1}
\end{aligned}
$$

which completes the induction step. (Here, the second $\sim_{\mathcal{P}}$ is from induction assumption, the fourth one is from $s=1$ case, and the others are from Lemma 8.3.) 
8.3. Proof of Proposition 5.5(B), We argue by induction on $l=|c|$. First suppose that $l=1$. If $a_{1}$ is in Case I, then it should be in Case I(b) by assumption and the result is trivial. If $a_{1}$ is in Case II, then the only possible case is when $a_{1}$ is in Case II(b) and either $a_{1}$ is not processed in the same step as $a_{2}$ or $\alpha=\left(a_{1}\right)$. Again the result is trivial in this case.

From now on suppose that $l \geq 2$ and the result holds up to $l-1$. Note that $a_{1}<c_{2}$ since otherwise $a_{1} \rightarrow_{\mathcal{P}} c_{1}$ by Condition ( $\pitchfork$ ). If $a_{1}<c_{1}$, then $a_{1}$ is in Case I(b) and $a_{1}$ bumps $c_{1}$. Then $a_{1} \leftarrow_{\mathcal{P}} a_{j}$ for any $j \in[2, m]$ and $a_{i} \leftarrow_{\mathcal{P}} c_{j}$ for $j \in[2, l]$, and thus we may apply induction assumption on $\left(a_{m}, \ldots, a_{2}\right)$ and $\left(c_{l}, \ldots, c_{2}\right)$ to prove the claim. Thus it suffices to assume that $c_{1}<a_{1}<c_{2}$. Since $a_{1} \dashv_{\mathcal{P}} c_{1}$, it implies that $a_{1} \rightarrow \mathcal{P} c_{1}$, and thus $a_{1}$ is in Case II. Suppose that $a_{1}$ is in Case II(b) and $a_{1}, \ldots, a_{k}$ are processed in the same step but $a_{k+1}$ is not. As $a_{1} \leftarrow \mathcal{P} \cdots \leftarrow \mathcal{P} a_{k}$, it means that $a_{1}, \ldots, a_{k}$ bump $c_{1}, \ldots, c_{k}$, respectively, and we may apply induction hypothesis to $\left(a_{m}, \ldots, a_{k+1}\right)$ and $\left(c_{l}, \ldots, c_{k+1}\right)$ similarly to above.

It remains to assume that $a_{1}$ is in Case II(a). Since $a_{1} \leftarrow \mathcal{P} a_{2}$, this only happens when $a_{1} \leftrightarrow-\mathcal{P} c_{2}$. However, as $a_{1} \rightarrow \mathcal{P} a_{2}$ and $a_{2} f_{\mathcal{P}} c_{2}$, this forces that $a_{1}<c_{2}<a_{2}$ and $a_{2} \rightarrow \mathcal{P} c_{2}$ by Condition ( $\pitchfork)$. In other words, $\left\{c_{1}, a_{1}, c_{2}, a_{2}\right\}$ is a ladder in $\mathcal{P}$. Thus by the assumption that $a_{1}$ is in Case II(a) we have $a_{2} t^{--\mathcal{P}} c_{3}$. We may iterate this argument and observe that $\left\{c_{1}, a_{1}, c_{2}, a_{2}, \ldots, c_{l}, a_{l}\right\}$ is a ladder. However, in this case $a_{1}$ is in Case II(b), which is a contradiction. Thus $a_{1}$ cannot be in Case II(a) and the claim is proved.

8.4. Proof of Proposition 5.5](C), We assume that the input is given by $\left(\alpha=\left(a_{m}, \ldots, a_{1}\right), c=\right.$ $\left.\left(c_{l}, \ldots, c_{1}\right)\right)$ and the output is given by $(-, \beta)$ where $\beta=\left(b_{m}, \ldots, b_{1}\right)$. Furthermore, we suppose that $a_{i}$ is processed in the first step of the algorithm. For example, if $a_{i}$ is in Case I(a) or Case I(b) then it means that $a_{j}=\infty$ for $j<i$.

In order to prove (C), we need to show that $b_{i} \neq \infty$ and $b_{i} \leftarrow_{\mathcal{P}} b_{i+1}$ under the assumption that $a_{i}, a_{i+1} \neq \infty, a_{i} \leftarrow \mathcal{P} a_{i+1}$, and $b_{i+1} \neq \infty$. First we consider the case when $a_{i}$ and $a_{i+1}$ are processed in the same step. If this step is in Case II(a), then $a_{i}=b_{i}$ and $a_{i+1}=b_{i+1}$ thus the result is obvious. On the other hand, if this step is in Case II(b) then one may easily observe that $b_{i}<a_{i}<b_{i+1}<a_{i+1}$ and $\left\{b_{i}, a_{i}, b_{i+1}, a_{i+1}\right\}$ is a ladder in $\mathcal{P}$. In particular we have $b_{i} \leftarrow_{\mathcal{P}} b_{i+1}$ as well.

Therefore, it suffices to assume that $a_{i}$ and $a_{i+1}$ are processed in different steps. We let $\mathfrak{d}=$ $\left(d_{l^{\prime}}, \ldots, d_{1}\right)$ be the chain obtained after the first step (which processes $\left.a_{i}\right)$ is performed. (See Figure 26]) For example, if $a_{i}$ is in Case I(a) then $l^{\prime}=l+1, d_{l^{\prime}}=a_{i}$, and $d_{j}=c_{j}$ for $j \in[1, l]$.

First, we note that $b_{i} \neq \infty$; otherwise, $a_{i}$ is in Case I(a) which means that $a_{i}$ becomes the largest element in the chain $\mathfrak{d}$. Since $a_{i} \leftarrow \mathcal{P} a_{i+1}$, it means that $a_{i+1}$ is also in Case I(a), which contradicts the assumption that $b_{i+1} \neq \infty$. In particular, it follows that $l=l^{\prime}$, i.e. the length of $c$ and $\mathfrak{d}$ should be equal.

It remains to show that $b_{i} \leftarrow \mathcal{P} b_{i+1}$. First assume that $a_{i}$ is in Case $\mathrm{I}(\mathrm{b})$ ), i.e. there exists $j \in[0, l-1]$ such that $c_{j}<a_{i}<c_{j+1}$ and $c_{j} \leftarrow_{\mathcal{P}} a_{i}$. (Here we put $c_{0}=-\infty$ as before.) In this case $b_{i}=c_{j+1}, d_{j+1}=a_{i}$, and $d_{k}=c_{k}$ for $k \neq j+1$. Now, if $a_{i+1}$ is in

- Case I(a): this is impossible as we assumed that $b_{i+1} \neq \infty$.

- Case I(b): $a_{i+1}$ should bump $d_{k}=c_{k}$ for some $k \geq j+2$ since $d_{j+1}=a_{i} \leftarrow \mathcal{P} a_{i+1}$. As a result, $b_{i}=c_{j+1} \leftarrow \mathcal{P} c_{k}=b_{i+1}$. 


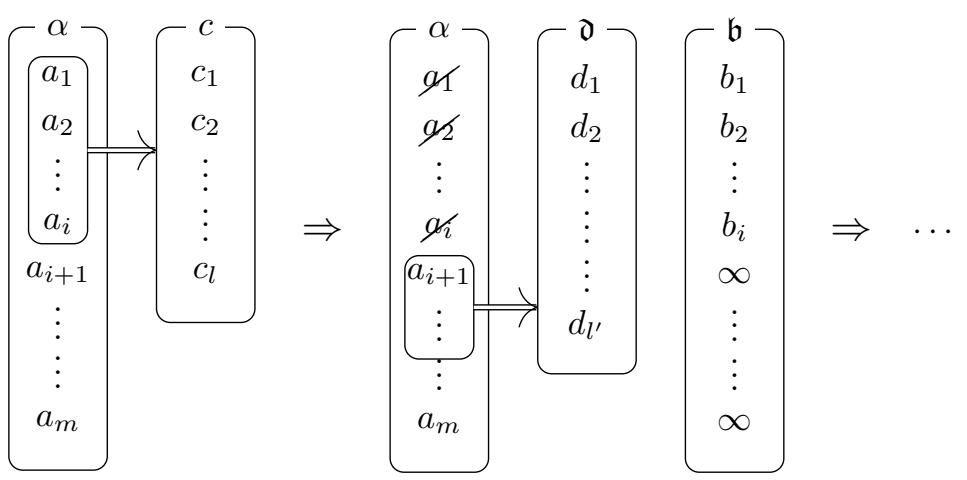

FIGURE 26. Description of the calculation

- Case II(a): since $a_{i} \leftarrow_{\mathcal{P}} a_{i+1}$, this is only possible when there exists $d_{k}=c_{k}$ for some $k \geq j+2$ such that $c_{k} \leftarrow_{-\mathcal{P}} a_{i+1}$. As $c_{j+1} \leftarrow_{\mathcal{P}} c_{k}$, we have $c_{j+1} \leftarrow_{\mathcal{P}} a_{i+1}$ by Condition ( $\left.\pitchfork\right)$ applied to $\left(c_{j+1}, c_{k}, a_{i+1}\right)$. Therefore we have $b_{i}=c_{j+1} \leftarrow \mathcal{P} a_{i+1}=b_{i+1}$.

- Case II(b): it is shown in the same way as Case II(a).

Now we assume that $a_{i}$ is in Case II(a), i.e. $a_{i}=b_{i}$ and $d=\mathfrak{d}$. Then for any $x \geq a_{i+1}$ we have $a_{i}=b_{i} \leftarrow_{\mathcal{P}} x$ by Condition ( $\left.\pitchfork\right)$ applied to $\left(a_{i}, a_{i+1}, x\right)$, and thus the only nontrivial case occurs when $a_{i+1}$ is in Case II(b). However, this is only possible when there exists $j \in[1, l]$ such that $a_{i}{ }^{--\mathcal{P}} c_{j}{ }^{--\mathcal{P}} a_{i+1}$, in which case $a_{i}$ and $a_{i+1}$ should be processed in the same step because of the maximality in Case II. This violates the aforementioned assumption.

Lastly we assume that $a_{i}$ is in Case II(b), i.e. $a_{i} \rightarrow \mathcal{P} b_{i}$. Again, by Condition ( $\pitchfork$ ) the only nontrivial case occurs when $a_{i+1}$ is in Case II(b) and this is only possible when there exists $j \in[1, l]$ such that $a_{i} \leftarrow \mathcal{P} c_{j}=d_{j} \leftarrow--\mathcal{P} a_{i+1}$. In this case we have $b_{i+1}=c_{j}$ and thus $b_{i} \leftarrow \mathcal{P} c_{j}=b_{i+1}$ by Condition ( $\pitchfork)$ applied to $\left(b_{i}, a_{i}, c_{j}\right)$.

We exhaust all the possibilities and thus completed the proof of $(\mathrm{C})$

8.5. Proof of Proposition 5.5](D), We keep the setup in the proof of(C) above. First we consider the case when $a_{i}$ and $a_{i+1}$ are processed in the same step. If this step is in Case II(a), then $a_{i}=b_{i}$ and $a_{i+1}=b_{i+1}$ thus the result is obvious. On the other hand, if this step is in Case II(b) then one may easily observe that $b_{i}<a_{i}=b_{i+1}<a_{i+1}$ and $\left\{b_{i}, a_{i}=b_{i+1}, a_{i+1}\right\}$ is a ladder in $\mathcal{P}$. In particular we have $b_{i} \leftarrow_{--\mathcal{P}} b_{i+1}$ as well. Therefore, it suffices to assume that $a_{i}$ and $a_{i+1}$ are processed in different steps.

We suppose that $b_{i+1}=\infty$, i.e. $a_{i+1}$ is in Case I(a). Since $a_{i} \psi_{\mathcal{P}} a_{i+1}$, it follows that $d_{l^{\prime}} \neq a_{i}$, i.e. $l=l^{\prime}$ and $d_{l}=c_{l}$ (and also $l \neq 0$ ). However, it is only possible when $a_{i}<c_{l}$ and $c_{l} \leftarrow \mathcal{P} a_{i+1}$, which implies $a_{i} \leftarrow \mathcal{P} a_{i+1}$ by Condition ( $\left.\pitchfork\right)$ applied to $\left(a_{i}, c_{l}, a_{i+1}\right)$. This violates our assumption, and thus $b_{i+1} \neq \infty$ as expected.

It remains to show that $b_{i} \psi_{\mathcal{P}} b_{i+1}$ if $b_{i} \neq \infty$, i.e. the length of $c$ is equal to $\mathfrak{d}$. We first assume that $a_{i}$ is in Case I(b), i.e. there exists $j \in[0, l-1]$ such that $c_{j}<a_{i}<c_{j+1}$ and $c_{j} \leftarrow \mathcal{P} a_{i}$. (Here we put $c_{0}=-\infty$ as before.) In this case we have $b_{i}=c_{j+1}>a_{i}, d_{j+1}=a_{i}$, and $d_{k}=c_{k}$ for any $k \neq j+1$. As $b_{i} \nvdash_{\mathcal{P}} a_{i+1}$ by Condition ( $\left.\pitchfork\right)$ applied to $\left(a_{i}, b_{i}=c_{j+1}, a_{i+1}\right)$, it follows that $b_{i} \nvdash_{\mathcal{P}} x$ for any $x \leq a_{i+1}$ again by Condition $(\pitchfork)$ applied to $\left(b_{i}, x, a_{i+1}\right)$. Therefore the only nontrivial case 
occurs when $a_{i+1}$ is in Case I(b) as well. This is only possible when either [there exists $k \in[0, j-1$ ] such that $c_{k}<a_{i+1}<c_{k+1}$ and $\left.c_{k} \leftarrow \mathcal{P} a_{i+1}\right]$ or $\left[c_{j}<a_{i+1}<d_{j+1}=a_{i}\right.$ and $\left.c_{j} \leftarrow \mathcal{P} a_{i+1}\right]$. Thus $b_{i+1}$ is equal to either $c_{k+1}$ for $k<j$ or $a_{i}$. In either case, we have $b_{i}=c_{j+1} \nvdash_{\mathcal{P}} b_{i+1}$.

Let us assume that $a_{i}$ is in Case II(a), i.e. $c=\mathfrak{d}$. As above, the only nontrivial case occurs when $a_{i+1}$ is in Case I(b), i.e. there exists $j \in[0, l-1]$ such that $c_{j}<a_{i+1}<c_{j+1}$ and $c_{j} \leftarrow \mathcal{P} a_{i+1}$, in which case $b_{i+1}=c_{j+1}$. Now if $a_{i}=b_{i} \leftarrow_{\mathcal{P}} b_{i+1}=c_{j+1}$, then as $a_{i}$ is in Case II(a) there exists $k<j+1$ such that $a_{i} \leftarrow_{-\mathcal{P}} c_{k}$. But this is contradiction since $c_{j} \leftarrow \mathcal{P} a_{i+1}$ implies $c_{k} \leftarrow \mathcal{P} a_{i+1}$, which means $a_{i} \leftarrow \mathcal{P} a_{i+1}$ by Condition ( $\left.\pitchfork\right)$ applied to $\left(a_{i}, c_{k}, a_{i+1}\right)$.

Lastly, we assume that $a_{i}$ is in Case II(b) so that $a_{i} \rightarrow \rightarrow \mathcal{P} b_{i}$. As $a_{i}$ and $a_{i+1}$ are not processed in the same step, $a_{i}$ should be in $\mathfrak{d}$. If $a_{i+1}$ is in

- Case I(a): this is impossible as we assumed that $b_{i+1}=\infty$.

- Case I(b): since $a_{i} \nvdash_{\mathcal{P}} a_{i+1}, a_{i+1}$ either bumps $a_{i}$ or some element above $a_{i}$ in the chain $\mathfrak{d}$, i.e. $b_{i+1}=a_{i}$ or $b_{i+1} \leftarrow_{\mathcal{P}} a_{i}$. In either case, we should have $b_{i} \nvdash_{\mathcal{P}} b_{i+1}$ since otherwise $a_{i} \rightarrow_{\mathcal{P}} b_{i}$.

- Case II(a): suppose that $b_{i} \leftarrow \mathcal{P} b_{i+1}=a_{i+1}$. As $b_{i} \leftarrow^{--\mathcal{P}} a_{i}$, we have $a_{i}<a_{i+1}$ by Condition ( $\pitchfork$ ) applied to $\left(b_{i}, a_{i}, a_{i+1}\right)$, and thus $\left\{b_{i}, a_{i}, a_{i+1}\right\}$ is a ladder in $\mathcal{P}$. However, this violates the maximality of Case II as $a_{i}$ and $a_{i+1}$ are not processed in the same step. Thus we should have $b_{i} \psi_{\mathcal{P}} b_{i+1}=a_{i+1}$

- Case II(b): $b_{i+1}$ is some element in the chain $d^{\prime}$ satisfying $a_{i+1} \rightarrow_{\mathcal{P}} b_{i+1}$. If $b_{i} \leftarrow \mathcal{P} b_{i+1}$, then we should have $b_{i}<a_{i}<b_{i+1}<a_{i+1}$ by Condition ( $\left.\pitchfork\right)$ applied to $\left(b_{i}, a_{i}, b_{i+1}\right)$ and also $b_{i} \leftarrow \mathcal{P} a_{i+1}$ by Condition ( $\pitchfork)$ applied to $\left(b_{i}, b_{i+1}, a_{i+1}\right)$. However, in such a case $\left\{b_{i}, a_{i}, a_{i+1}\right\}$ is a ladder in $\mathcal{P}$, which violates the maximality in Case II. Thus we have $b_{i} \nvdash_{\mathcal{P}} b_{i+1}$

We exhaust all the possibilities and thus completed the proof of (D).

8.6. Proof of Proposition 5.6. Hereafter we write $\widehat{\infty}=\infty$ and $\widehat{a}:=n+1-a$ for $a \in[1, n]$. ( $n$ is assumed to be fixed.) We define a new order $\widehat{\mathcal{P}}$ on $[1, n]$ such that $a \rightarrow_{\widehat{\mathcal{P}}} b$ if and only if $\widehat{a} \leftarrow_{\mathcal{P}} \widehat{b}$. Then one can easily check that $\mathcal{P}=\mathcal{P}_{\lambda, m}$ if and only if $\widehat{\mathcal{P}}=\mathcal{P}_{\lambda^{\prime}, m}$. Also, from now on we write $\mathfrak{C}^{\mathcal{P}}, \mathfrak{C}^{\widehat{\mathcal{P}}}, \Phi^{\mathcal{P}}, \Psi_{X}^{\widehat{\mathcal{P}}}$, etc. to clarify which partial order is used in their definitions. We define

$$
\begin{aligned}
& \widehat{\bullet}: \mathfrak{A} \rightarrow \mathfrak{A}: \alpha=\left(a_{m}, \ldots, a_{1}\right) \mapsto \widehat{\alpha}=\left(\widehat{a_{1}}, \ldots, \widehat{a_{m}}\right) \\
& \widehat{\bullet}: \mathfrak{C}^{\mathcal{P}} \rightarrow \mathfrak{C}^{\widehat{\mathcal{P}}}: c=\left(c_{l}, \ldots, c_{1}\right) \mapsto \widehat{c}=\left(\widehat{c_{1}}, \ldots, \widehat{c_{l}}\right)
\end{aligned}
$$

and $\widehat{\bullet}: \widehat{\mathfrak{C}^{\mathcal{P}}} \rightarrow \mathfrak{C}^{\mathcal{P}}$ similarly. Also we set

$$
\omega: \mathfrak{C}^{\mathcal{P}} \mathfrak{A} \rightarrow \mathfrak{A C}^{\widehat{\mathcal{P}}}:(c, \alpha) \mapsto(\widehat{\alpha}, \widehat{c}),
$$

and $\omega: \mathfrak{C}^{\widehat{\mathcal{P}}} \mathfrak{A} \rightarrow \mathfrak{A C}^{\mathcal{P}}$ similarly. (Here we abuse notations and denote both functions by $\omega$.) Note that these functions are well-defined bijections.

Suppose that $\Phi^{\mathcal{P}}(\alpha, c)=(d, \beta)$ for some $(\alpha, c) \in \mathfrak{A C}^{\mathcal{P}}$ and $(d, \beta) \in \mathfrak{C}^{\mathcal{P}} \mathfrak{A}$ where $\alpha=\left(a_{m}, \ldots, a_{1}\right)$ and $\beta=\left(b_{m}, \ldots, b_{1}\right)$. We set $X=\left\{m+1-i \mid i \in[1, m], a_{i} \neq \infty, b_{i}=\infty\right\}$. (This set records in which step Case $\mathrm{I}(\mathrm{a})$ occured when calculating $\Phi^{\mathcal{P}}(\alpha, c)$.) We claim the following.

Lemma 8.4. Keep the assumptions above. Then we have $(\alpha, c)=\left(\omega \circ \Psi_{X}^{\widehat{P}} \circ \omega \circ \Phi^{\mathcal{P}}\right)(\alpha, c)=$ $\left(\omega \circ \Psi_{X}^{\widehat{P}} \circ \omega\right)(d, \beta)$, i.e. $(\widehat{c}, \widehat{\alpha})=\Psi_{X}^{\widehat{P}}(\widehat{\beta}, \widehat{d})$. 
Remark. Since the set $X$ depends on $(\alpha, c)$, the lemma above does not show that $\omega \circ \Psi_{X}^{\widehat{P}} \circ \omega \circ \Phi^{\mathcal{P}}$ is the identity. However, it shows that one can recover $(\alpha, c)$ from $(d, \beta)$ and $X$, which essentially proves Proposition 5.6.

Proof. We may assume that $m \geq 1$ and $a_{m} \neq \infty$. Suppose that it only needs one step. If it is in

- Case $\mathrm{I}(\mathrm{a})$ : in this case it is clear that $\alpha=\left(a_{1}\right), \beta=(\infty), d=\left(a_{1}\right)+c$, and $X=\{1\}$. Thus $\Psi_{X}^{\widehat{P}}(\widehat{\beta}, \widehat{d})=\Psi_{\{1\}}^{\widehat{\mathcal{P}}}\left((\infty), \widehat{c}+\left(\widehat{a_{1}}\right)\right)=\left(\widehat{c},\left(\widehat{a_{1}}\right)\right)=(\widehat{c}, \widehat{\alpha})$ as desired. (This corresponds to Case $\infty$ (b) of $\mathbf{A l g}_{\Psi} \cdot$ )

- Case I(b): we have $\alpha=\left(a_{1}\right)$ and $X=\emptyset$. There exists $r \in[0, l-1]$ such that $c_{r}<a_{1}<c_{r+1}$ and $c_{r} \leftarrow \mathcal{P} a_{1}$. (If $a_{1}<c_{1}$ then we set $r=0$.) Then we have $\beta=\left(c_{r+1}\right)$ and $d=$ $\left(c_{l}, \ldots, c_{r+2}, a_{1}, c_{r}, \ldots, c_{1}\right)$. Since $\widehat{c_{r+2}}<\widehat{c_{r+1}}<\widehat{a_{1}}$ and $\widehat{c_{r+2}} \leftarrow \widehat{\widehat{p}} \widehat{c_{r+1}}$, we have $\Psi_{X}^{\widehat{P}}(\widehat{\beta}, \widehat{d})=$ $\Phi^{\widehat{\mathcal{P}}}\left(\left(\widehat{c_{r+1}}\right),\left(\widehat{c_{1}}, \ldots, \widehat{c_{r}}, \widehat{a_{1}}, \widehat{c_{r+2}}, \ldots, \widehat{c_{l}}\right)\right)=\left(\left(\widehat{c_{1}}, \ldots, \widehat{c_{r}}, \widehat{c_{r+1}}, \widehat{c_{r+2}}, \ldots, \widehat{c_{l}}\right),\left(\widehat{a_{1}}\right)\right)=(\widehat{c}, \widehat{\alpha})$ as desired. (This corresponds to Case I(b) of $\mathbf{A l g}_{\Psi}$, and it is still valid when $r+2=l+1$.)

- Case II(a): we have $X=\emptyset, \alpha=\beta, c=d$, and $a_{1}<a_{2}<\cdots<a_{m}$. There exists $r, h$ such that $\left\{c_{r}, \ldots, c_{r+h}\right\} \cup \underline{\alpha}$ is a ladder in $\mathcal{P}$ and $c_{r}<a_{i}<c_{r+h}$ for any $i$. Then it is easy to see that $\widehat{a_{m}}<\cdots<\widehat{a_{2}}<\widehat{a_{1}},\left\{\widehat{c_{r}}, \ldots, \widehat{c_{r+h}}\right\} \cup \underline{\widehat{\alpha}}$ is a ladder in $\widehat{\mathcal{P}}$, and $\widehat{c_{r+h}}<\widehat{a_{i}}<\widehat{c_{r}}$ for any $i$. Thus $\Psi_{X}^{\widehat{P}}(\widehat{\beta}, \widehat{d})=\Phi^{\widehat{\mathcal{P}}}(\widehat{\alpha}, \widehat{c})=(\widehat{c}, \widehat{\alpha})$ as desired. (This corresponds to Case II(a) of $\mathbf{A l g}_{\Psi} \cdot$ )

- Case II(b): we have $X=\emptyset$. As in the description of $\mathbf{A l g}_{\Phi}$, we choose $r, h$ and $0=$ $u(r-1)<u(r)<u(r+1)<\cdots<u(r+h)=m$ such that $c_{i}<a_{u(i-1)+1}<\cdots<a_{u(i)}$ for $i \in[r, r+h]$ and $\left\{c_{r}, \ldots, c_{r+h}, a_{1}, \ldots, a_{m}\right\}$ is a ladder in $\mathcal{P}$. Then it follows that $d=\left(c_{l}, \ldots, c_{r+h+1}, a_{u(r+h)}, \ldots, a_{u(r)}, c_{r-1}, \ldots, c_{1}\right)$ and

$$
b_{j}=\left\{\begin{array}{lr}
c_{i} & \text { if } j=u(i-1)+1 \text { for some } i \in[r, r+h], \\
a_{j-1} & \text { otherwise. }
\end{array}\right.
$$

However, it implies that $\widehat{b_{1}}>\widehat{b_{2}}>\cdots>\widehat{b_{m}},\left\{\widehat{a_{u(r)}}, \ldots, \widehat{a_{u(r+h)}}, \widehat{b_{1}}, \ldots, \widehat{b_{m}}\right\}$ is a ladder in $\widehat{\mathcal{P}}$, and $\widehat{b_{u(i-1)}+1}=\widehat{c_{i}}>\widehat{a_{u(i-1)}+1}>\cdots>\widehat{a_{u(i)}}$ for $i \in[r, r+h]$. Also note that $\widehat{c_{r-1}} \rightarrow_{\widehat{\mathcal{P}}} x$ for any $x \in\left\{\widehat{a_{u(r)}}, \ldots, \widehat{a_{u(r+h)}}, \widehat{b_{1}}, \ldots, \widehat{b_{m}}\right\}$ when $r>1$. Thus

$$
\begin{aligned}
\Psi_{X}^{\widehat{P}}(\widehat{\beta}, \widehat{d}) & =\Phi^{\widehat{\mathcal{P}}}\left(\left(\widehat{b_{1}}, \ldots, \widehat{b_{m}}\right),\left(\widehat{c_{1}}, \ldots, \widehat{c_{r-1}}, \widehat{a_{u(r)}}, \ldots, \widehat{\left.a_{u(r+h}\right)}, \widehat{c_{r+h+1}} \ldots, \widehat{c_{l}}\right)\right) \\
& =\left(\left(\widehat{c_{1}}, \ldots, \widehat{c_{r-1}}, \widehat{c_{r}}, \ldots, \widehat{c_{r+h}}, \widehat{c_{r+h+1}}, \ldots, \widehat{c_{l}}\right),\left(\widehat{a_{1}}, \ldots, \widehat{a_{m}}\right)\right)=(\widehat{c}, \widehat{\alpha})
\end{aligned}
$$

as desired. (This corresponds to Case II(b) of $\mathbf{A} \lg _{\Psi}$.)

To prove the general case we argue by induction on the number of steps of $\mathbf{A l g}_{\Phi}$ for the calculation of $\Phi^{\mathcal{P}}(\alpha, c)$. Let $m^{\bullet} \in[0, m-1]$ be the smallest element such that $a_{m} \bullet+1, \ldots, a_{m}$ are processed in the same step. Set $\alpha^{\bullet}=\left(a_{m} \bullet, \ldots, a_{1}\right), \alpha^{\dagger}=\left(a_{m}, \ldots, a_{m} \bullet+1\right), \beta^{\bullet}=\left(b_{m} \bullet, \ldots, b_{1}\right)$ and $\beta^{\dagger}=\left(b_{m}, \ldots, b_{m} \bullet+1\right)$, so that $\alpha=\alpha^{\dagger}+\alpha^{\bullet}$ and $\beta=\beta^{\dagger}+\beta^{\bullet}$. Then there exists $d^{\bullet} \in \mathfrak{C}^{\mathcal{P}}$ such that $\Phi^{\mathcal{P}}\left(\alpha^{\bullet}, c\right)=\left(d^{\bullet}, \beta^{\bullet}\right)$ and $\Phi^{\mathcal{P}}\left(\alpha^{\dagger}, d^{\bullet}\right)=\left(d, \beta^{\dagger}\right)$ because of the choice of $m^{\bullet}$. Thus by induction assumption we have $\left(\widehat{c}, \widehat{\alpha^{\bullet}}\right)=\Psi_{X}^{\widehat{P}}\left(\widehat{\beta^{\bullet}}, \widehat{d^{\bullet}}\right)$ and $\left(\widehat{d^{\bullet}}, \widehat{\alpha^{\dagger}}\right)=\Psi_{X^{\dagger}}^{\widehat{\mathcal{P}}}\left(\widehat{\beta^{\dagger}}, \widehat{d}\right)$ where $X^{\bullet}=\left\{i+m^{\bullet}-m \mid i \in\right.$ $\left.X \cap\left[m-m^{\bullet}+1, m\right]\right\}$ and $X^{\dagger}=X \cap\left[1, m-m^{\bullet}\right]$.

Therefore, in order to prove the lemma, it suffices to show that the first step of the calculation of $\Psi_{X}^{\widehat{P}}(\widehat{\beta}, \widehat{d})$ processes $\widehat{b_{m} \bullet+1}, \ldots, \widehat{b_{m}}$ but not $\widehat{b_{m} \bullet}$. (See Figure 27) If $a_{m}$ is in Case I of $\mathbf{A l g}_{\Phi}$ then $\widehat{b_{m}}$ is in Case I or Case $\infty$ of $\mathbf{A l g}_{\Psi}$ as shown above, in which case the statement is trivial. Thus 
we assume that $a_{m}$ is in Case II of $\mathbf{A l g}_{\Phi}$. Then we have $|d|=\left|d^{\bullet}\right|$ and $X^{\dagger}=\emptyset$. Thus in particular $\Psi_{X^{\dagger}}^{\widehat{\mathcal{P}}}\left(\widehat{\beta^{\dagger}}, \widehat{d}\right)=\Phi^{\widehat{\mathcal{P}}}\left(\widehat{\beta^{\dagger}}, \widehat{d}\right)$. We set $d=\left(d_{q}, \ldots, d_{1}\right)$ and $d^{\bullet}=\left(d_{q}^{\bullet}, \ldots, d_{1}^{\bullet}\right)$.

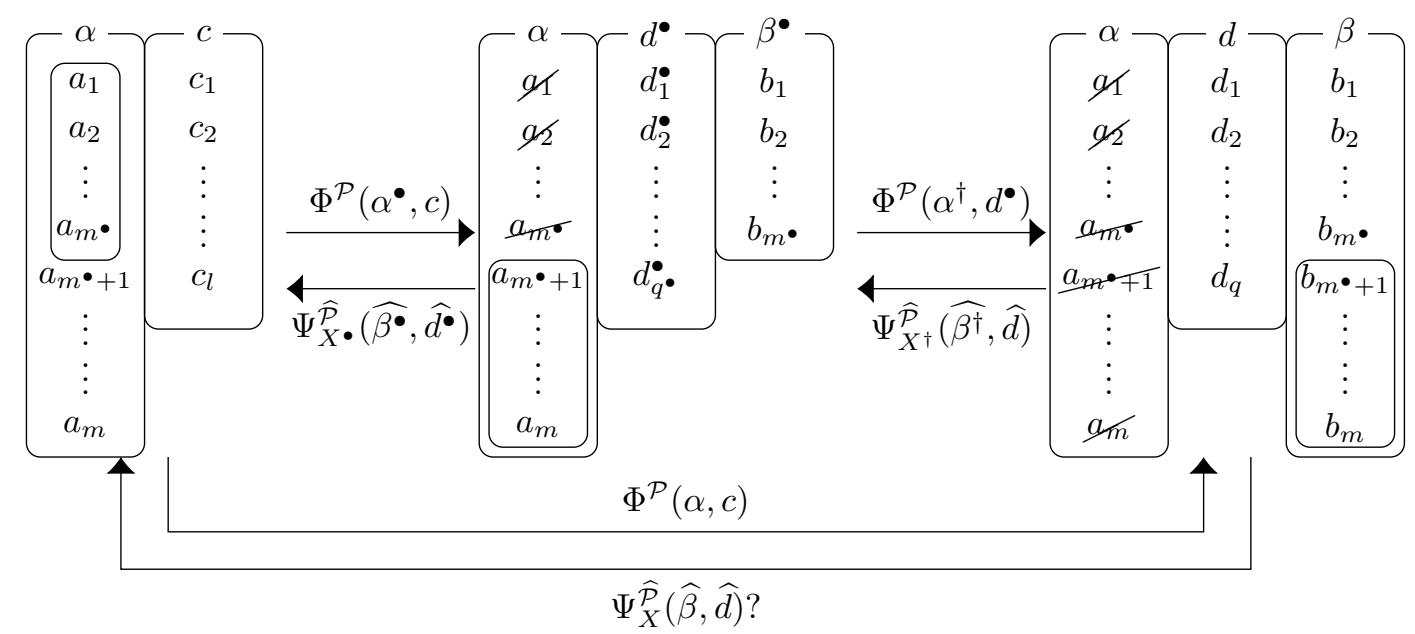

FiguRE 27. Strategy of the proof

Case II(a). Suppose that $a_{m}$ is in Case II(a) of $\mathbf{A l g}_{\Phi}$. Then $d=d^{\bullet}, \beta^{\dagger}=\alpha^{\dagger}$ and there exist $r, h$ such that $\mathcal{L}:=\left\{d_{r}^{\bullet}, \ldots, d_{r+h}^{\bullet}, a_{m} \cdot+1, \ldots, a_{m}\right\}$ is a ladder in $\mathcal{P}$ and $d_{r}^{\bullet} \leq x \leq d_{r+h}^{\bullet}$ for any $x \in \mathcal{L}$. Here we need to check that $\left\{\widehat{b_{m} \bullet}\right\} \cup\{\widehat{x} \mid x \in \mathcal{L}\}$ is not a ladder in $\widehat{\mathcal{P}}$, i.e. either $m^{\bullet}=0$ or $b_{m} \bullet$ does not satisfy both $b_{m} \bullet \leftarrow_{\mathcal{P}} b_{m} \bullet+1$ and $b_{m} \bullet \leftarrow-\mathcal{P} d_{r}^{\bullet}$. For the sake of contradiction we suppose otherwise. By Proposition [5.5](C) it also means that $a_{m} \bullet \leftarrow_{\mathcal{P}} a_{m} \bullet+1$. Also $a_{m} \bullet$ cannot be in Case $\mathrm{I}(\mathrm{a})$ of $\mathbf{A} \lg _{\Phi}$.

Suppose that $a_{m} \bullet$ is in Case I(b) of $\mathbf{A l g}_{\Phi}$. Then $b_{m}^{\bullet}$ is bumped out from the chain $\mathfrak{d}$, and thus $d_{r}^{\bullet} f^{\prime} \rightarrow \mathcal{P} b_{m} \bullet$ unless $a_{m} \bullet$ bumps the $r$-th element of $\mathfrak{d}$ and $a_{m} \bullet=d_{r}^{\bullet}$. But this is absurd since it means that $a_{m} \bullet<b_{m} \bullet$ but $b_{m} \bullet \leftarrow-\mathcal{P}^{\bullet} d_{r}^{\bullet}=a_{m} \bullet$.

Now we assume that $a_{m} \bullet$ is in Case II(a) of $\operatorname{Alg}_{\Phi}$ and $\mathcal{L}^{\prime}:=\left\{d_{r^{\prime}}^{\bullet}, \ldots, d_{r^{\prime}+h^{\prime}}^{\bullet}, a_{m^{\prime}}, \ldots, a_{m} \bullet\right\}$ is the corresponding ladder in $\mathcal{P}$ where $d_{r^{\prime}}^{\bullet}<\cdots<d_{r^{\prime}+j}^{\bullet}$ and $d_{r^{\prime}}^{\bullet}<a_{m^{\prime}}<\cdots<a_{m} \bullet<d_{r^{\prime}+h^{\prime}}^{\bullet}$ Then $b_{m} \bullet=a_{m} \bullet$, and thus $d_{r}^{\bullet-\rightarrow} b_{m} \bullet=a_{m} \bullet$ which is only possible when $r^{\prime}+h^{\prime}=r$, i.e. $\mathcal{L} \cup \mathcal{L}^{\prime}$ is again a ladder in $\mathcal{P}$. (Note that $a_{m} \bullet=b_{m}^{\bullet} \leftarrow \mathcal{P} a_{m} \bullet+1$.) However, it contradicts the minimality of $m^{\bullet}$.

Assume that $a_{m} \bullet$ is in Case II(b) of $\mathbf{A l g}_{\Phi}$. Then $a_{m} \bullet=d_{i}^{\bullet}$ for some $i$, and as $a_{m} \bullet \leftarrow \mathcal{P} a_{m} \bullet+1$

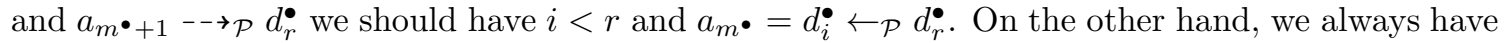
$a_{m} \bullet-\rightarrow \mathcal{P} b_{m} \bullet$, and thus $d_{r}^{\bullet} \rightarrow_{\mathcal{P}} b_{m} \bullet$ by Condition $(\pitchfork)$. However, it contradicts the assumption that $d_{r}^{\bullet-\rightarrow} b_{m} \bullet$.

Case II(b). Suppose that $a_{m}$ is in Case II(b) of $\mathbf{A l g}_{\Phi}$ and let $\mathcal{L}:=\left\{d_{r}^{\bullet}, \ldots, d_{r+h}^{\bullet}, a_{m} \bullet+1, \ldots, a_{m}\right\}$ be the corresponding ladder so that $d_{r}^{\bullet} \leq x \leq a_{m}$ for any $x \in \mathcal{L}$. Then direct calculation as above shows that $\mathcal{L}=\left\{d_{r}, \ldots, d_{r+h}, b_{m} \bullet+1, \ldots, b_{m}\right\}$. Note that $\mathcal{L} \cup\left\{d_{r-1}^{\bullet}\right\}$ cannot be a ladder, and thus in order for $\widehat{b_{m} \bullet}$ to be processed with $\widehat{b_{m} \bullet+1}, \ldots, \widehat{b_{m}}$ we should have that $\{\widehat{x} \mid x \in \mathcal{L}\} \cup\left\{\widehat{b_{m} \bullet}\right\}$ is a 
ladder in $\widehat{\mathcal{P}}$, i.e. $b_{m} \bullet+1-\rightarrow_{\mathcal{P}} b_{m} \bullet$ and $x \rightarrow_{\mathcal{P}} b_{m} \bullet$ for any $x \in \mathcal{L}-\left\{b_{m} \bullet+1\right\}$ by maximality in Case II. By Proposition [5.5(D)] it also means that $a_{m} \bullet \leftarrow_{-\mathcal{P}} a_{m} \bullet+1$.

Assume that $a_{m} \bullet$ is in Case I(b) of $\mathbf{A l g}_{\Phi}$. Then $a_{m} \bullet=d_{i}^{\bullet}$ for some $i$. Since $a_{m} \bullet{ }^{--_{\mathcal{P}}} a_{m} \bullet+1$ and $d_{r}^{\bullet}{ }^{\bullet} \mathcal{P} a_{m} \bullet+1$, we should have $r=i$ and $a_{m} \bullet=d_{r}^{\bullet}$. But it contradicts that $b_{m} \bullet>a_{m} \bullet$ as $b_{m} \bullet \leftarrow-\mathcal{P} b_{m} \bullet+1=d_{r}^{\bullet}$.

Assume that $a_{m} \bullet$ is in Case II(a) of $\mathbf{A l g}_{\Phi}$. Then $b_{m} \bullet=a_{m} \bullet \leftarrow^{-\mathcal{P}} a_{m+1} \neq b_{m} \bullet+1$ which contradicts that $x \rightarrow_{\mathcal{P}} b_{m} \bullet$ for any $x \in \mathcal{L}-\left\{b_{m} \bullet+1\right\}$.

Assume that $a_{m} \bullet$ is in Case II(b) of $\mathbf{A l g}_{\Phi}$ and $\mathcal{L}^{\prime}$ is the corresponding ladder where $a_{m} \bullet>b_{m} \bullet>$

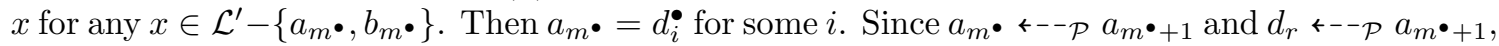
it follows that $r=i$. Together with the fact that $x \rightarrow_{\mathcal{P}} b_{m} \bullet$ for any $x \in \mathcal{L}-\left\{b_{m} \bullet+1\right\}$, it follows that $\mathcal{L} \cup \mathcal{L}^{\prime}$ is again a ladder. However, it contradicts the minimality of $m^{\bullet}$.

We exhaust all the cases and finish the proof.

We are ready to prove Proposition 5.6 .

Proof of Proposition 5.6. Recall that we have $\left(\alpha=\left(a_{m}, \ldots, a_{1}\right), c\right),\left(\alpha^{\prime}=\left(a_{m}^{\prime}, \ldots, a_{1}^{\prime}\right), c^{\prime}\right) \in \mathfrak{A C}$ such that $a_{i}=\infty \Leftrightarrow a_{i}^{\prime}=\infty$ and $\Phi(\alpha, c)=\Phi\left(\alpha^{\prime}, c^{\prime}\right)$. Let $(d, \beta) \in \mathfrak{C A}$ be such a result. Let $X=\left\{m+1-i \mid i \in[1, m], a_{i} \neq \infty, b_{i}=\infty\right\}=\left\{m+1-i \mid i \in[1, m], a_{i}^{\prime} \neq \infty, b_{i}=\infty\right\}$. Then by Lemma 8.4 we have $\omega\left(\Psi_{X}^{\widehat{P}}(\widehat{\beta}, \widehat{d})\right)=(\alpha, c)=\left(\alpha^{\prime}, c^{\prime}\right)$ as desired.

\section{Proof of Theorem 6.1 and 4.13}

9.1. Preliminary lemmas. Before the proof we first state the following series of lemmas which will be frequently used later on.

Lemma 9.1. Suppose that $\left\{a_{1}, \ldots, a_{k}\right\}$ is a ladder in $\mathcal{P}$ where $k \geq 2$ and $a_{1}<\cdots<a_{k}$.

(1) If $b \rightarrow \mathcal{P} a_{k}$, then either $\left\{a_{1}, \ldots, a_{k-1}, a_{k}, b\right\}$ or $\left\{a_{1}, \ldots, a_{k-2}, a_{k-1}, b\right\}$ is a ladder in $\mathcal{P}$.

(2) If $b{ }_{--\mathcal{P}} a_{1}$, then either $\left\{b, a_{1}, a_{2}, \ldots, a_{k}\right\}$ or $\left\{b, a_{2}, a_{3}, \ldots, a_{k}\right\}$ is a ladder in $\mathcal{P}$.

Proof. For (1), first note that we have $b \rightarrow_{\mathcal{P}} a_{k-2}$ which follows from Condition ( $\left.\pitchfork\right)$ as $a_{k-2} \leftarrow \mathcal{P} a_{k}$. Now if $b \rightarrow \mathcal{P} a_{k-1}$, then $\left\{a_{1}, \ldots, a_{k-1}, a_{k}, b\right\}$ is a ladder in $\mathcal{P}$. Otherwise, we have $b \rightarrow \rightarrow_{\mathcal{P}} a_{k-1}$ and thus $\left\{a_{1}, \ldots, a_{k-2}, a_{k-1}, b\right\}$ is a ladder in $\mathcal{P}$. (2) is proved similarly.

Lemma 9.2. Suppose that $\left\{a_{1}, \ldots, a_{k}\right\}$ is a ladder in $\mathcal{P}$ where $a_{1}<\cdots<a_{k}$. If $x \notin\left\{a_{1}, \ldots, a_{k}\right\}$, $a_{1} \leftarrow \mathcal{P} x$, and no one is climbing a ladder in $\mathcal{P}$, then $a_{i} \leftarrow \mathcal{P} x$ and $a_{i+1}<x$ for $i \leq k-3$.

Proof. Since no one is climbing a ladder in $\mathcal{P}$, we have $a_{k} f_{>} \mathcal{P} x$. Since $a_{k-2} \leftarrow_{\mathcal{P}} a_{k}$, this means that $a_{k-2}<x$ by Condition ( $\pitchfork$ ). Now suppose that there exists $i \leq k-3$ such that $a_{i} \nvdash_{\mathcal{P}} x$ (and thus $k \geq 5$ ). By Condition ( $\pitchfork)$, it is equivalent to assuming that $a_{k-3} \leftarrow_{-\mathcal{P}} x$. Since $a_{k-3} \leftarrow \mathcal{P} a_{k-1}$, this means that $x<a_{k-1}$ by Condition ( $\left.\pitchfork\right)$. Thus we have $x \leftarrow--\mathcal{P} a_{k-1}, a_{k}$ and $x-\rightarrow_{\mathcal{P}} a_{k-2}, a_{k-3}$. Since $a_{k-4} \leftarrow \mathcal{P} a_{k-2}$, we also have $x \rightarrow_{\mathcal{P}} a_{k-4}$ by Condition ( $\left.\pitchfork\right)$. Now one may check that $\mathcal{P}$ restricted to $\left\{a_{k-4}, a_{k-3}, a_{k-2}, x, a_{k}\right\}$ is isomorphic to $\mathcal{P}_{(3,1,1), 5}$, which is a contradiction. It remains to check that $a_{i}<x$ for $i \leq k-2$, and thus suppose otherwise (and thus $k \geq 4$ ). It implies that $a_{i-1} \leftarrow \mathcal{P} a_{i}$ by Condition ( $\pitchfork$ ) since $a_{i-1} \leftarrow \mathcal{P} x$ by above (or by assumption if $i=2$ ), which is a contradiction. 
Lemma 9.3. Suppose that $\left\{b_{1}, \ldots, b_{k}, a_{1}, a_{2}, a_{3}\right\}$ is a ladder in $\mathcal{P}$ where $b_{1}<\cdots<b_{k}<a_{1}<a_{2}<$ $a_{3}, x \notin\left\{b_{1}, \ldots, b_{k}, a_{1}, a_{2}, a_{3}\right\}, b_{1} \leftarrow \mathcal{P} x$, and no one is climbing a ladder in $\mathcal{P}$. Then the relation between $\left\{a_{1}, a_{2}\right\}$ and $x$ falls into one of the following. (Also see Figure 28.)

(9.3.1) $a_{1} \leftrightarrow-\mathcal{P} x$ and $a_{2} \rightarrow \mathcal{P} x$

(9.3,2) $a_{1} \leftarrow-\mathcal{P} x$ and $a_{2} \leftarrow--\mathcal{P} x$

(9.3.3) $a_{1} \leftarrow \mathcal{P} x$ and $a_{2} \leftarrow-\mathcal{P} x$

(9.3.4) $a_{1} \leftarrow \mathcal{P} x$ and $a_{2} \leftarrow \mathcal{P} x$

Proof. By Lemma 9.2 we may assume that $k=1$. Then one may check case-by-case using Condition

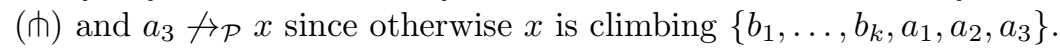

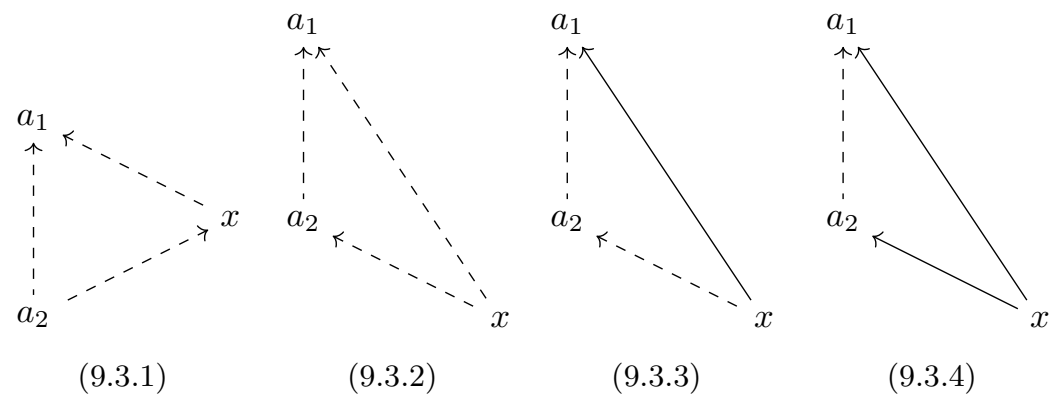

Figure 28. Possible cases in Lemma 9.3

Lemma 9.4. Suppose that $\left\{a_{1}, \ldots, a_{k}\right\}$ is a ladder in $\mathcal{P}$ where $a_{1}<\cdots<a_{k}$ and $k \geq 4, x \notin$ $\left\{a_{1}, \ldots, a_{k}\right\}$, and no one is climbing a ladder in $\mathcal{P}$. If $a_{2} \leftarrow^{--\mathcal{P}} x$, then it falls into one of the following cases. (Also see Figure 29)

(9.4.1) $a_{1} \leftrightarrow-\mathcal{P} x, a_{3} \rightarrow \mathcal{P} x$

(9.4.2) $k=4, a_{1} \leftarrow \mathcal{P} x, a_{3} \rightarrow \mathcal{P} x, a_{4} \rightarrow \mathcal{P} x$

(9.4.3) $k=4, a_{1} \leftarrow \mathcal{P} x, a_{3} \leftarrow_{-\mathcal{P}} x, a_{4} \rightarrow \mathfrak{p} x$

Proof. Using Lemma 9.2. Condition ( $\pitchfork)$, and the assumption that $x$ is not climbing $\left\{a_{1}, \ldots, a_{k}\right\}$ in $\mathcal{P}$, one may deduce that the only possibilities are the above three cases and possibly $[k=5$, $\left.a_{1} \leftarrow \mathcal{P} x, a_{3} \leftarrow-\mathcal{P} x, a_{4} \rightarrow_{\mathcal{P}} x, a_{5}-\rightarrow \mathcal{P} x\right]$ (see the last diagram in Figure 29). However, the latter is impossible since in this case $a_{3}$ is climbing the ladder $\left\{a_{1}, a_{2}, x, a_{5}\right\}$.

9.2. Proof of Theorem 6.1 (A), We are ready to prove Theorem 6.1 (A) Since each column of $P T$ (resp. $Q T$ ) is a chain with respect to $\mathcal{P}$ (resp. the usual order), we may restrict our attention to comparing elements in two adjacent columns of $P T$ and $Q T$, respectively. To this end, we set $\alpha, \beta \in \mathfrak{A}$ to be $\alpha=\left(a_{m}, a_{m-1}, \ldots, a_{1}\right), \beta=\left(b_{m}, \ldots, b_{1}\right)$ and set $P T=\left(P T_{1}, P T_{2}\right)$ where $P T_{1}, P T_{2} \in \mathfrak{C}$ are defined to be $P T_{1}=\left(d_{p}, \ldots, d_{1}\right)$ and $P T_{2}=\left(e_{q}, \ldots, e_{1}\right)$. We assume that $\Phi(\alpha, \emptyset)=\left(P T_{1}, \beta\right), \Phi(\beta, \emptyset)=\left(P T_{2}, \gamma\right)$ for some $\gamma \in \mathfrak{A}$. We define $Q T=\left(Q T_{1}, Q T_{2}\right)$ to be as in the algorithm of $\mathcal{P}$-RS.

We argue by induction on $m=|\alpha|$. There is nothing to prove when its length is 0 , and thus suppose that $m \geq 1$ and the statement is true up to $m-1$. We set $m \bullet<$ (specified 


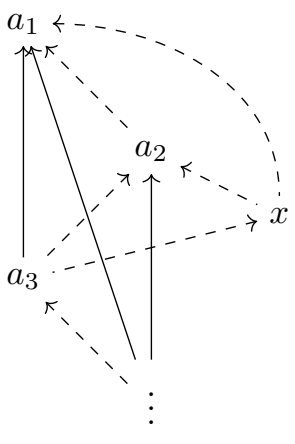

(9.4.1)

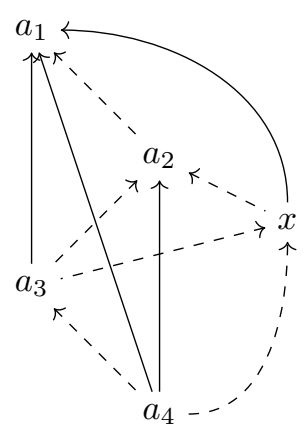

(9.4,2)

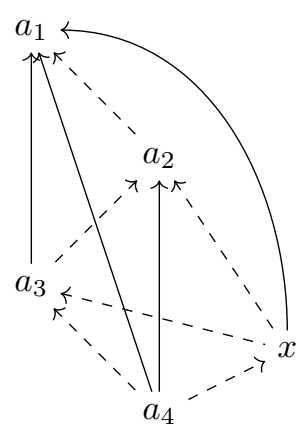

(9.4.3)

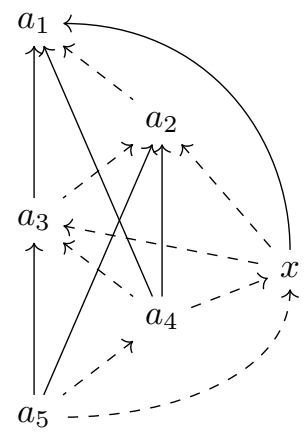

not possible

Figure 29. (Im)Possible cases in Lemma 9.4

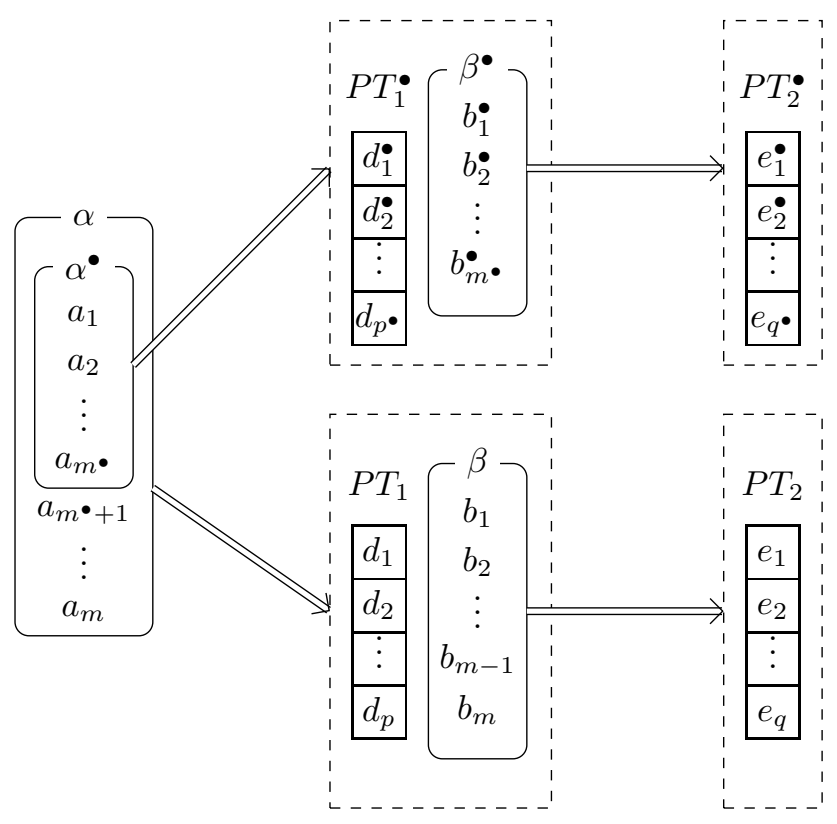

FIGURE 30. Setup for induction argument

later $)$, and define $\alpha^{\bullet}=\left(a_{m} \bullet, \ldots, a_{1}\right), \beta^{\bullet}=\left(b_{m}^{\bullet}, \ldots, b_{2}^{\bullet}, b_{1}^{\bullet}\right), P T^{\bullet}=\left(P T_{1}^{\bullet}, P T_{2}^{\bullet}\right)$ where $P T_{1}^{\bullet}=$ $\left(d_{p^{\bullet}}^{\bullet}, \ldots, d_{2}^{\bullet}, d_{1}^{\bullet}\right), P T_{2}^{\bullet}=\left(e_{q}^{\bullet}, \ldots, e_{2}^{\bullet}, e_{1}^{\bullet}\right), Q T^{\bullet}=\left(Q T_{1}^{\bullet}, Q T_{2}^{\bullet}\right)$, and $\gamma^{\bullet} \in \mathfrak{A}$ analogously. (See Figure 30])

It suffices to assume that $a_{m} \neq \infty$. For $a_{m}$ in Case $\mathrm{I}(\mathrm{a})$, we set $m^{\bullet}=m-1$. Then we have $p=p^{\bullet}+1, q=q^{\bullet}, P T_{1}=\left(a_{m}\right)+P T_{1}^{\bullet}, Q T_{1}=(m)+Q T_{1}^{\bullet}, P T_{2}=P T_{2}^{\bullet}$, and $Q T_{2}=Q T_{2}^{\bullet}$. In this case there is nothing to prove. From now on, we divide all the remaining possibilities into the following cases. (Note that we have $p=p^{\bullet}$ and $Q T_{1}=Q T_{1}^{\bullet}$ in these cases.) 
I. $a_{m}$ is in Case I(b) (see Figure 31): we set $m^{\bullet}:=m-1$. There exists $\varrho \in[1, p]$ such that $d_{\varrho-1}^{\bullet}<a_{m}<d_{\varrho}^{\bullet}$ and $d_{\varrho-1}^{\bullet} \leftarrow \mathcal{P} a_{m}$. (Here we set $d_{0}^{\bullet}=-\infty$.) Here it is easy to observe that $\Phi\left(\left(a_{m}\right), \stackrel{P}{P} T_{1}^{\bullet}\right)=\left(P T_{1},\left(b_{m}\right)\right)$. Also we have $d_{\varrho}=a_{m}<d_{\varrho}^{\bullet}, d_{k}=d_{k}^{\bullet}$ if $k \neq \varrho$, $\beta^{\bullet}=\left(b_{m-1}, \ldots, b_{2}, b_{1}\right)$, and $b_{m}=d_{\varrho}^{\bullet}$.

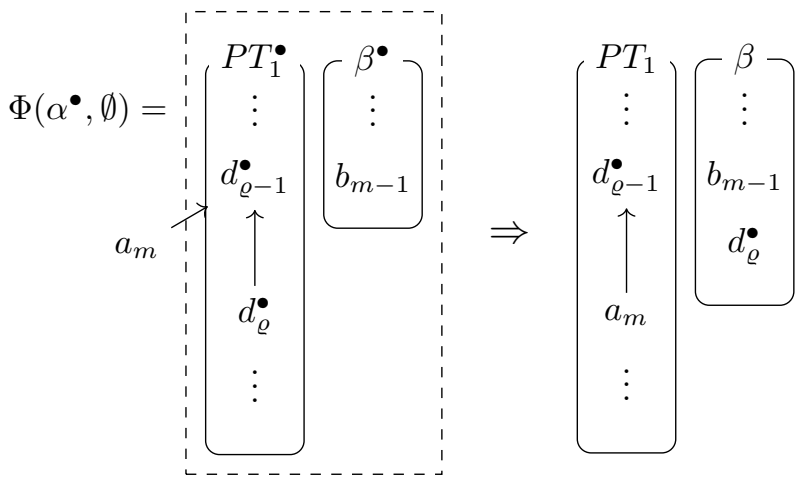

Figure 31. I. $a_{m}$ is in Case $\mathrm{I}(\mathrm{b})$

II. $a_{m}$ is in Case II(a) (see Figure [32): we set $m^{\bullet} \in[0, m-1]$ to be the smallest integer such that $a_{m} \bullet+1, \ldots, a_{m}$ are processed in the same step and $a_{i}{ }^{--\mathcal{P}} a_{i+1}$ if $i \in\left[m^{\bullet}+1, m-1\right]$. Then,

- $\Phi\left(\left(a_{m}, \ldots, a_{m} \bullet+1\right), P T_{1}^{\bullet}\right)=\left(P T_{1},\left(b_{m}, \ldots, b_{m} \bullet+1\right)\right)$,

- there exists $\varrho \in[1, p-1]$ such that $d_{\varrho}<a_{m} \bullet+1<\cdots<a_{m}<d_{\varrho+1}$, and

$-\mathcal{L}_{a d}:=\left\{d_{\varrho}, a_{m} \bullet+1, \cdots, a_{m}, d_{\varrho+1}\right\}$ is a ladder in $\mathcal{P}$.

Then we have $P T_{1}=P T_{1}^{\bullet}, \beta^{\bullet}=\left(b_{m} \bullet, \ldots, b_{2}, b_{1}\right)$, and $b_{i}=a_{i}$ for $i \in\left[m^{\bullet}+1, m\right]$.

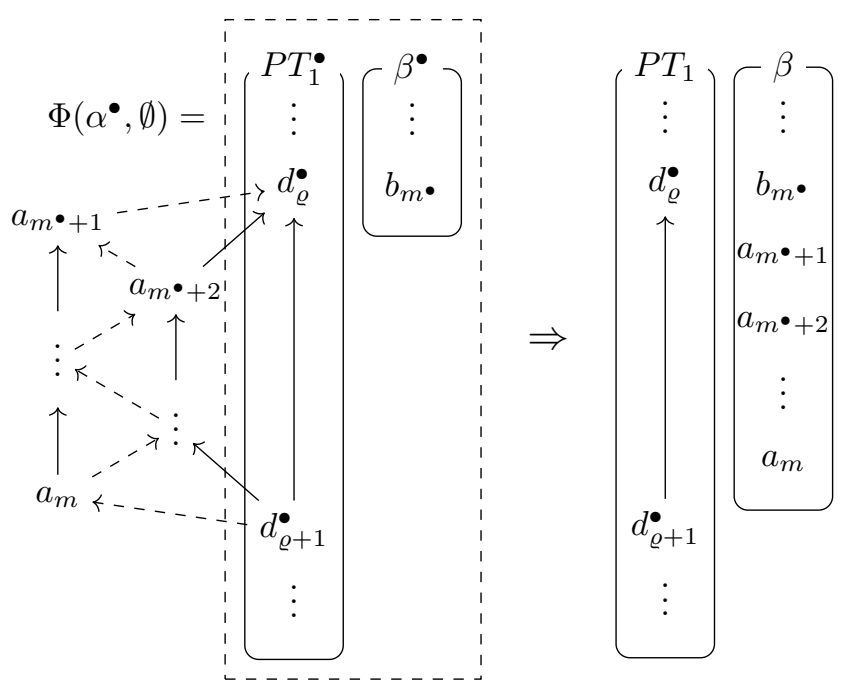

Figure 32. II. $a_{m}$ is in Case II(a) 
III. $a_{m}$ is in Case II(b) (see Figure 33): we set $m^{\bullet} \in[0, m-1]$ to be the smallest integer such that $a_{m} \bullet+1, \ldots, a_{m}$ are processed in the same step and $a_{i} \leftarrow \mathcal{P} a_{i+1}$ if $i \in\left[m^{\bullet}+1, m-1\right]$. Then,

- $\Phi\left(\left(a_{m}, \ldots, a_{m} \bullet+1\right), P T_{1}^{\bullet}\right)=\left(P T_{1},\left(b_{m}, \ldots, b_{m} \bullet+1\right)\right)$,

- there exists $\varrho \in\left[m-m^{\bullet}, p\right]$ such that $d_{\sigma+1}^{\bullet}<a_{m}^{\bullet+1}<d_{\sigma+2}^{\bullet}<a_{m+2}<\cdots<d_{\varrho}^{\bullet}<a_{m}$ where $\sigma:=\varrho-m+m^{\bullet}$,

- either $\varrho=q^{\bullet}$ or $d_{\varrho+1} \rightarrow \mathcal{P} a_{m}$ (by maximality in Case II), and

- $\mathcal{L}_{a d}:=\left\{d_{\sigma+1}^{\bullet}, a_{m} \bullet+1, d_{\sigma+2}^{\bullet}, a_{m} \bullet+2, \ldots, d_{\varrho}^{\bullet}, a_{m}\right\}$ is a ladder in $\mathcal{P}$.

Then we have $d_{i}=d_{i}^{\bullet}$ for $i \in[1, \sigma] \cup[\varrho+1, p]$ and $d_{i}=a_{i+m-\varrho}$ for $i \in[\sigma+1, \varrho], \beta^{\bullet}=$ $\left(b_{m} \bullet, \ldots, b_{2}, b_{1}\right)$, and $b_{i}=d_{i+\varrho-m}^{\bullet}$ for $i \in\left[m^{\bullet}+1, m\right]$.

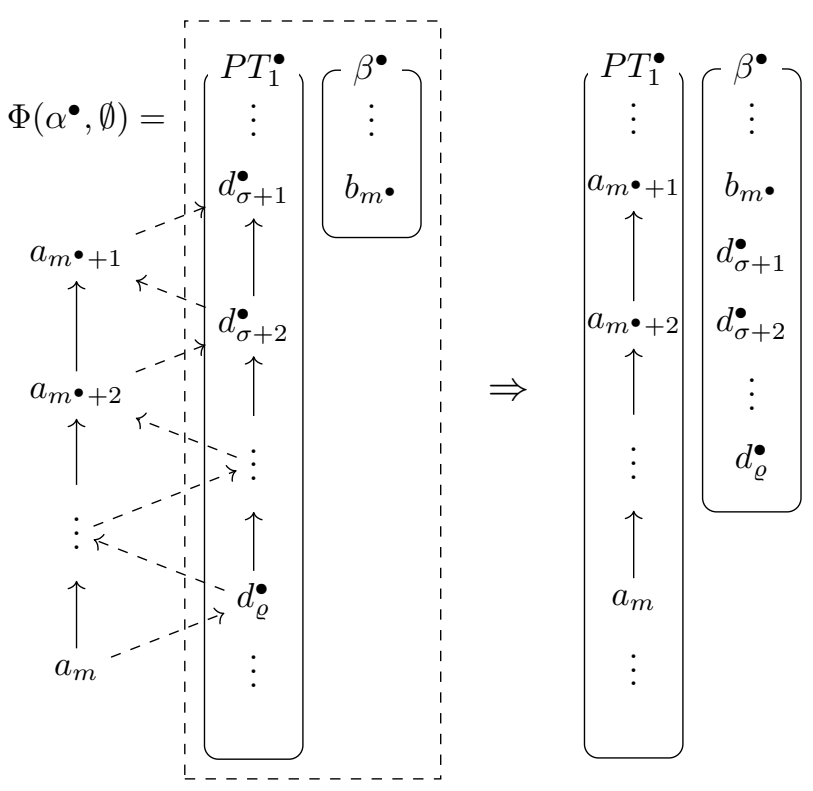

Figure 33. III. $a_{m}$ is in Case II(b)

The condition $\Phi\left(\left(a_{m}, \ldots, a_{m} \bullet+1\right), P T_{1}^{\bullet}\right)=\left(P T_{1},\left(b_{m}, \ldots, b_{m} \bullet+1\right)\right)$ implies that in each case $Q T_{2}$ is obtained from $Q T_{2}^{\bullet}$ by adding some entries in $\left[m^{\bullet}+1, m\right]$ in order, which in turn means that $Q T$ satisfies the standard Young tableau condition if and only if $p \geq q$. Thus in each case it suffices to prove that $p \geq q$ and $d_{i} t_{p_{\mathcal{P}}} e_{i}$ for $i \in[1, q]$. Now we also divide possibilities into following cases. (Here we use Lemma 5.4 in each case. Note that we always have $b_{m} \bullet+1<\cdots<b_{m}$.)

- $b_{m} \bullet+1$ is in Case I(a): we have $e_{q} \bullet \leftarrow_{\mathcal{P}} b_{i}$ for $i \in\left[m^{\bullet}+1, m\right]$. Thus $e_{i}=e_{i}^{\bullet}$ for $i \in\left[1, q^{\bullet}\right]$

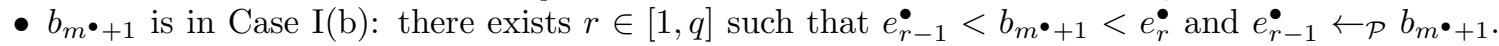
(Here we set $e_{0}^{\bullet}=-\infty$.) Then we have $e_{i}=e_{i}^{\bullet}$ for $i \in[1, r-1]$.

- $b_{m} \bullet+1$ is in Case II: let $m^{\prime} \in\left[1, m^{\bullet}+1\right]$ be the smallest integer such that $b_{m^{\prime}}, \ldots, b_{m} \bullet+1$ are processed in the same step (thus in particular $b_{m^{\prime}}<\cdots<b_{m} \bullet+1$ ), and we set $P T_{2}^{\circ}=\left(e_{q}^{\circ}, \ldots, e_{2}^{\circ}, e_{1}^{\circ}\right) \in$ $\mathfrak{C}$ to be " $P T_{2}$ right before the step processing $b_{m^{\prime}}, \ldots, b_{m}$ ", i.e. such that $\Phi\left(\left(b_{m}, \ldots, b_{m^{\prime}}\right), P T_{2}^{\circ}\right)=$ $\left(P T_{2},-\right)$ and also $\Phi\left(\left(b_{m} \bullet, \ldots, b_{m^{\prime}}\right), P T_{2}^{\circ}\right)=\left(P T_{2}^{\bullet},-\right)$. (As Case II does not increase the length of the chain, $P T_{2}^{\circ}$ is of length $q^{\bullet}$. See Figure 34) 


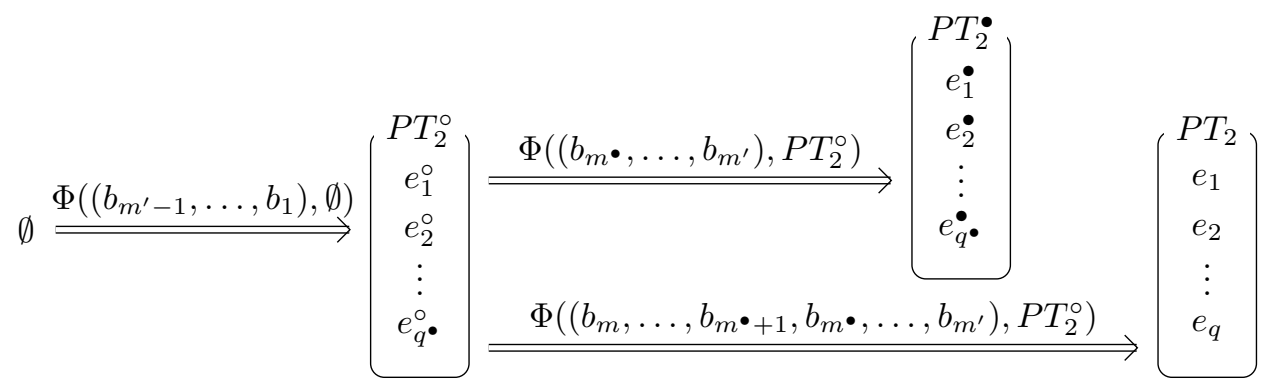

Figure 34. Definition of $P T_{2}^{\circ}, P T_{2}^{\bullet}$, and $P T_{2}$ when $b_{m} \bullet+1$ is in Case II

Also, we set $r, s \geq 1$ and $0 \leq m^{\prime}-1=u(r)<\cdots<u(r+s)=m^{\bullet}+1$ to be such that $e_{r+j}^{\circ}<b_{u(r+j-1)+1}<\cdots<b_{u(r+j)}$ for $j \in[1, s]$ and

$$
\mathcal{L}_{b e}:=\left\{e_{r+1}^{\circ}, \ldots, e_{r+s}^{\circ}, b_{m^{\prime}}, b_{m^{\prime}+1}, \ldots, b_{m} \bullet+1\right\}
$$

is a ladder in $\mathcal{P}$. Note that we always have $e_{i}=e_{i}^{\bullet}=e_{i}^{\circ}$ for $i \in[1, r]$ by Lemma 5.4 because $e_{r}^{\circ} \leftarrow_{\mathcal{P}} b_{j}$ for $j \in\left[m^{\prime}, m\right]$ by assumption. Also one may easily check that $e_{i}^{\bullet}=e_{i}^{\circ}$ for $i \geq r+s+1$. Here we list all the possibilities. (Also see Figure 35.)

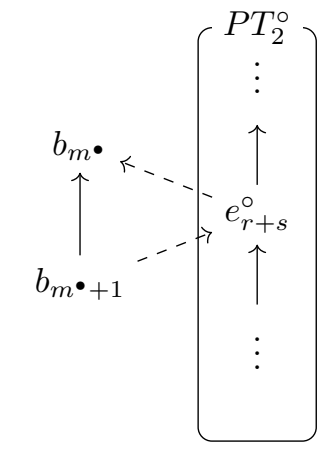

$u(r+s-1)=m^{\bullet}$

$b_{m} \bullet$ was in Case II(a)

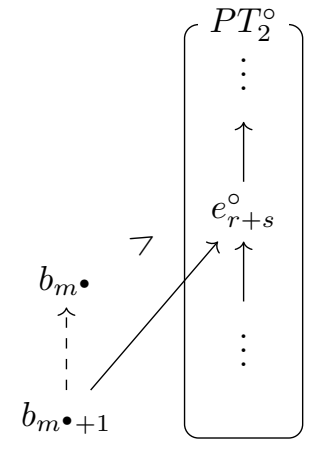

$u(r+s-1)<m^{\bullet}$

$b_{m} \bullet$ was in Case II (b)

FIgURE 35. Two possible cases of $b_{m} \bullet$ on the calculation of $\Phi\left(\left(b_{m} \bullet, \ldots, b_{m^{\prime}}\right), P T_{2}^{\circ}\right)$

○ Case II(a), $u(r+s-1)=m^{\bullet}$. (See Figure 36) This case includes $s=1$ and $u(r)+1=$ $u(r+1)=m^{\bullet}+1$. This means that $b_{m^{\prime}}, \ldots, b_{m} \bullet$ were originally in Case II(a) as well. We have $r+s+1 \leq q^{\bullet} \leq q, e_{i}^{\bullet}=e_{i}^{\circ}$ for all $i$ and also $e_{i}=e_{i}^{\bullet}$ for $i \in[r+1, r+s-1]$ by Lemma 5.4 because $e_{r+s-1}^{\bullet} \leftarrow_{\mathcal{P}} b_{j}$ for $j \in\left[m^{\bullet}+1, m\right]$.

- Case II(a), $u(r+s-1)<m^{\bullet}$. (See Figure 37) This means that $b_{m^{\prime}}, \ldots, b_{m} \bullet$ were originally in Case II(b). We have $r+s+1 \leq q^{\bullet} \leq q, e_{r+i}^{\bullet}=b_{u(r+i)}$ for $i \in[1, s-1], e_{r+s}^{\bullet}=b_{m} \bullet$, and $e_{i}=e_{i}^{\circ}$ for $i \in[r+1, r+s]$ by Lemma 5.4 because Case II(a) does not alter the given chain and $e_{r+s}^{\circ} \leftarrow_{\mathcal{P}} b_{j}$ for $j \in\left[m^{\bullet}+1, m\right]$ by assumption.

$\circ$ Case II(b), $u(r+s-1)<m^{\bullet}$. (See Figure 38,) This means that $b_{m^{\prime}}, \ldots, b_{m} \bullet$ were originally in Case II(b) as well. We have $e_{i}=e_{i}^{\bullet}$ for $i \in[r+1, r+s-1]$ and $e_{r+s}^{\bullet}=b_{m} \bullet$ by Lemma 5.4 since $e_{r+s-1}^{\bullet} \leftarrow_{\mathcal{P}} b_{j}$ for $j \in\left[m^{\bullet}+1, m\right]$ by assumption. 


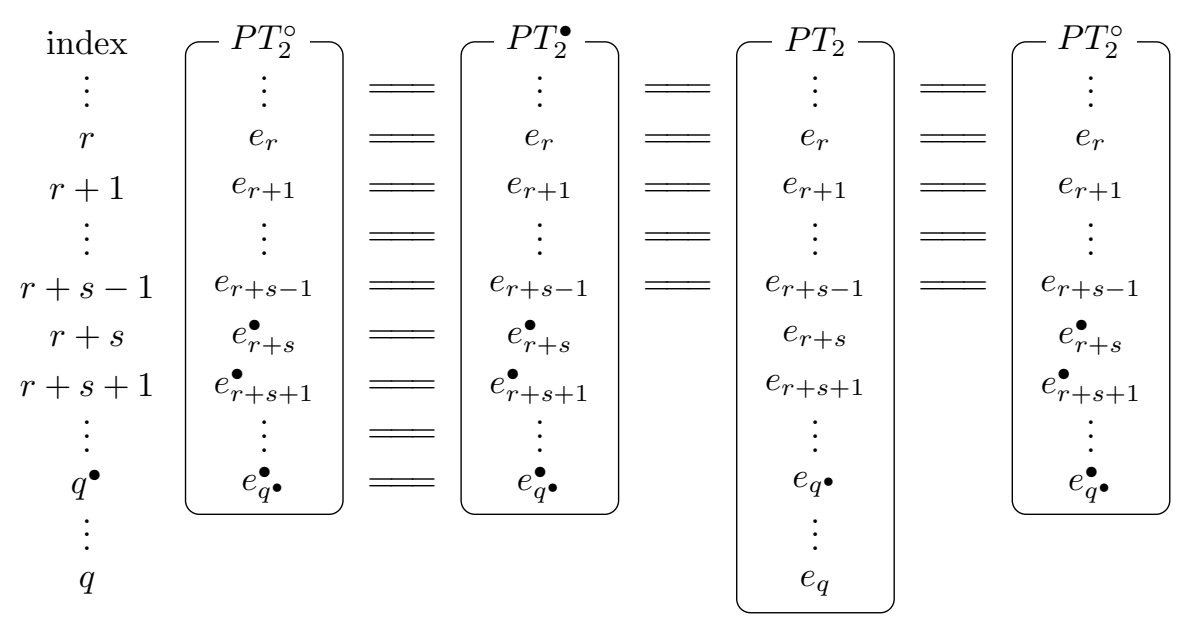

Figure 36. $P T_{2}^{\circ}, P T_{2}^{\bullet}$, and $P T_{2}$ when $b_{m} \bullet+1$ in Case II(a), $u(r+s-1)=m^{\bullet}$

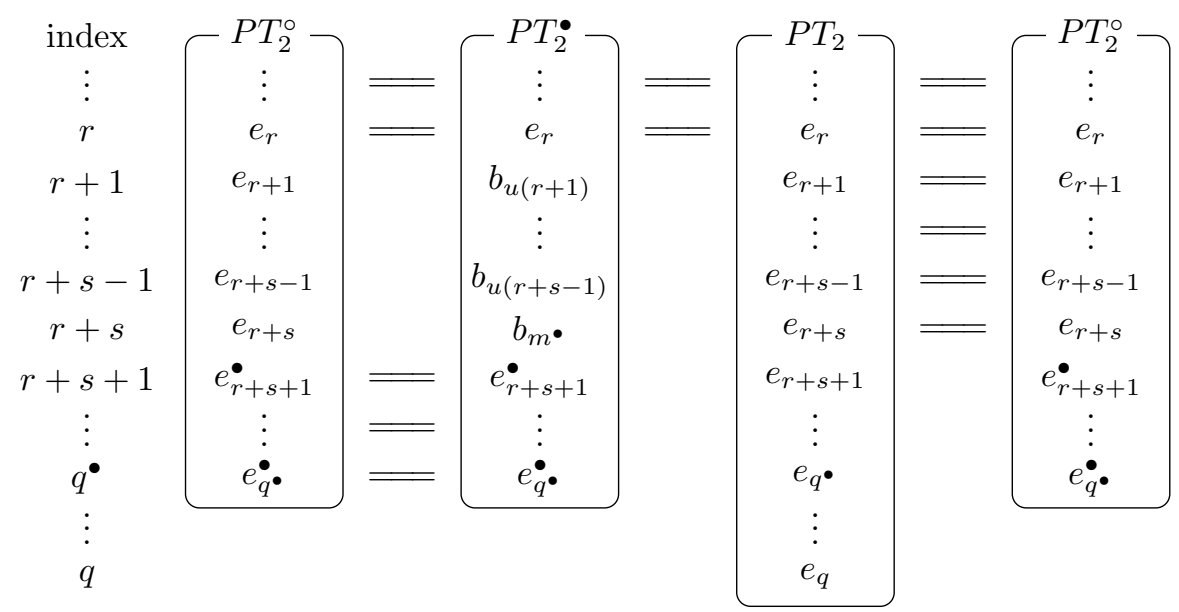

Figure 37. $P T_{2}^{\circ}, P T_{2}^{\bullet}$, and $P T_{2}$ when $b_{m} \bullet+1$ in Case II(a), $u(r+s-1)<m^{\bullet}$

○ Case II(b), $u(r+s-1)=m^{\bullet}$. (See Figure 39) This case includes $s=1$ and $u(r)+1=u(r+$ $1)=m^{\bullet}+1$. This means that $b_{m^{\prime}}, \ldots, b_{m} \bullet$ were originally in Case II(a). We have $e_{i}^{\bullet}=e_{i}^{\circ}$ for any $i$ and $e_{r+i}=b_{u(r+i)}$ for $i \in[1, s-1]$ by Lemma 5.4 because $b_{u(r+s-1)}=b_{m} \bullet \leftarrow \mathcal{P} b_{j}$ for $j \in\left[m^{\bullet}+1, m\right]$. Also, it is easy to observe that $e_{r+i}^{\bullet}<e_{r+i}$ for $i \in[1, s-1]$.

From now on we verify the conditions $p \geq q$ and $d_{i} t_{\mathcal{P}} e_{i}$ for $i \in[1, q]$ in each case. Note that $p=p^{\bullet} \geq q^{\bullet}$ and $d_{i}^{\bullet} \nrightarrow_{\mathcal{P}} e_{i}^{\bullet}$ for any $i$ by induction assumption, and thus $p \geq q$ if $q=q^{\bullet}$ and $d_{i} \not \supset_{\mathcal{P}} e_{i}$ if $d_{i}=d_{i}^{\bullet}, e_{i}=e_{i}^{\bullet}$. 


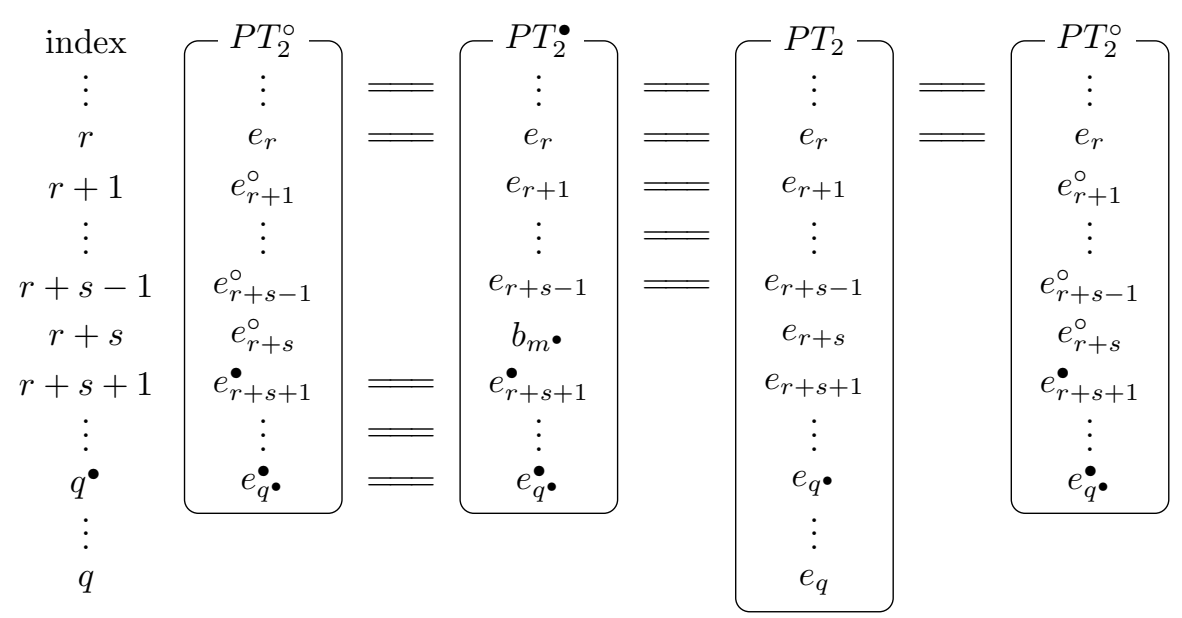

Figure 38. $P T_{2}^{\circ}, P T_{2}^{\bullet}$, and $P T_{2}$ when $b_{m} \bullet+1$ in Case $\mathrm{II}(\mathrm{b}), u(r+s-1)<m^{\bullet}$

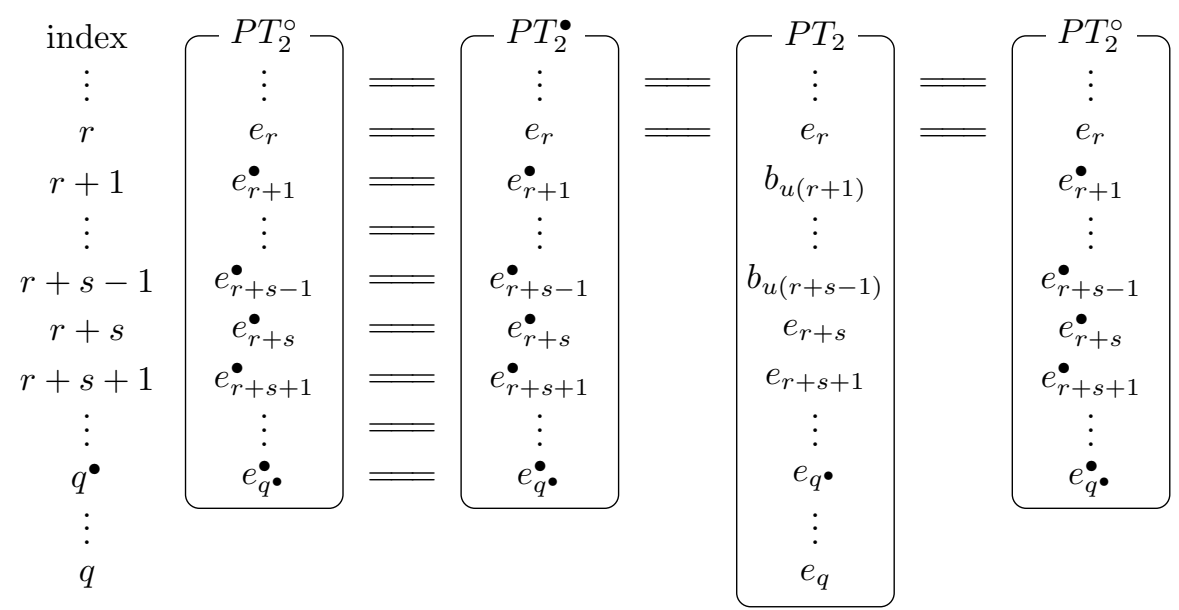

Figure 39. $P T_{2}^{\circ}, P T_{2}^{\bullet}$, and $P T_{2}$ when $b_{m} \bullet+1$ in Case II(b), $u(r+s-1)=m^{\bullet}$

I. $a_{m}$ is in Case I(b). We have $m^{\bullet}=m-1$. Here it suffices to check $d_{k} \nrightarrow_{\mathcal{P}} e_{k}$ only for $k$ satisfying

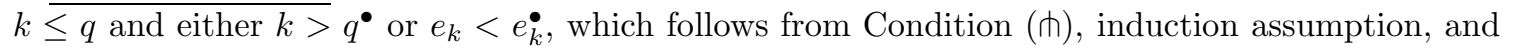
the fact that $d_{k} \leq d_{k}^{\bullet}$ for $k \in[1, p]$.

I.i. $b_{m}$ is in Case I(a): we have $q=q^{\bullet}+1$ and $P T_{2}=\left(b_{m}\right)+P T_{2}^{\bullet}$. First in this case $p \geq \varrho \geq q$ since $e_{q-1}^{\bullet}=e_{q}^{\bullet \bullet} \leftarrow_{\mathcal{P}} b_{m}=d_{\varrho}^{\bullet}$. Now it suffices to check $d_{q} \succ_{\mathcal{P}} e_{q}$. Now if $\varrho>q$, then $e_{q}=d_{\varrho}^{\bullet} \nvdash_{\mathcal{P}} d_{q}^{\bullet}=d_{q}$. If $\varrho=q$, then $e_{q}=d_{\varrho}^{\bullet} \nvdash_{\mathcal{P}} a_{m}=d_{\varrho}$.

I.ii. $b_{m}$ is in Case $\mathrm{I}(\mathrm{b})$ : we have $q=q^{\bullet}$, and $e_{i} \neq e_{i}^{\bullet}$ only when $i=r$ in which case $e_{r}=b_{m}=d_{\varrho}^{\bullet}$. Thus it suffices to check $d_{r} t_{\mathcal{P}} e_{r}$. Note that $r \leq \varrho$ since $d_{\varrho}^{\bullet}=b_{m} \rightarrow_{\mathcal{P}} e_{r-1}^{\bullet}$. If $r<\varrho$ then $d_{r}=d_{r}^{\bullet} f_{\mathcal{P}} d_{\varrho}^{\bullet}=e_{r}$. If $r=\varrho$ then $d_{\varrho}=a_{m} t_{\mathcal{P}} d_{\varrho}^{\bullet}=e_{r}$ thus the condition still holds. 
I.iii. $b_{m}$ is in Case $\mathrm{II}(\mathrm{a}), u(r+s-1)=m-1$ : we have $P T_{2}^{\bullet}=P T_{2}$, and thus there is nothing to check.

I.iv. $b_{m}$ is in Case II(a), $u(r+s-1)<m-1$ : in this case we have $q=q^{\bullet}, e_{r+s}^{\bullet}=b_{m-1}$, $e_{r+j}^{\bullet}=b_{u(r+j)}$ for $j \in[1, s-1]$, and $e_{k}^{\bullet}=e_{k}$ if $k \notin[r+1, r+s]$. Thus it suffices to check $d_{k} t_{\mathcal{P}} e_{k}$ for $k \in[r+1, r+s]$. Note that $r+s \leq \varrho$ since $e_{r+s}^{\bullet}=b_{m-1}<b_{m}=d_{\varrho}^{\bullet}$. First suppose that $k \in[r+1, r+s], k<\varrho$, and $d_{k} \rightarrow_{\mathcal{P}} e_{k}$. Then as $d_{k}=d_{k}^{\bullet} \leftarrow_{\mathcal{P}} d_{\varrho}^{\bullet}=b_{m}$ it implies that $d_{k}$ is climbing $\mathcal{L}_{b e}$ in $\mathcal{P}$, which is a contradiction. Now suppose $k \in[r+1, r+s], k \geq \varrho$ and $d_{k} \rightarrow_{\mathcal{P}} e_{k}$ (which forces that $k=\varrho=r+s$ ). Note that $\mathcal{L}_{b e} \cup\left\{e_{r+s+1}\right\}$ is a ladder in $\mathcal{P}$ since $b_{m}$ is in Case II(a) and $\mathcal{L}_{b e} \cup\left\{e_{r+s+1}, d_{\varrho+1}\right\}$ is also a ladder as $d_{\varrho+1}=d_{\varrho+1}^{\bullet} \rightarrow_{\mathcal{P}} d_{\varrho}^{\bullet}=b_{m}$ and $d_{\varrho+1}=d_{\varrho+1}^{\bullet} t_{\mathcal{P}} e_{r+s+1}^{\bullet}=e_{r+s+1}$. Since $d_{\varrho} \leftarrow \mathcal{P} d_{\varrho+1}$, we see that $d_{\varrho}$ is climbing the ladder $\mathcal{L}_{b e} \cup\left\{e_{r+s+1}, d_{\varrho+1}\right\}$ which is a contradiction. (See Figure 40,)

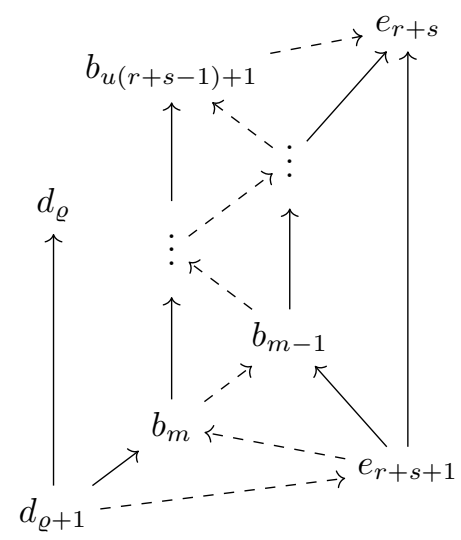

Figure 40. $a_{m}$ in Case I(b), $b_{m}$ in Case II(a), $u(r+s-1)<m-1, \varrho=r+s$

I.v. $b_{m}$ is in Case II(b), $u(r+s-1)<m-1$ : we have $q=q^{\bullet}, e_{r+s}=b_{m}>b_{m-1}=e_{r+s}^{\bullet}$ and $e_{k}=e_{k}^{\bullet}$ for $k \neq r+s$, and thus there is nothing to check.

I.vi. $b_{m}$ is in Case II(b), $u(r+s-1)=m-1$ : we have $q=q^{\bullet}, e_{r+i}^{\bullet}<b_{u(r+i)}=e_{r+i}$ for $i \in[1, s]$ and $e_{k}^{\bullet}=e_{k}$ for $k \notin[r+1, r+s]$, and thus there is nothing to check.

II. $a_{m}$ is in Case II(a). Recall that $b_{i}=d_{i+\varrho-m}^{\bullet}$ for $i \in\left[m^{\bullet}+1, m\right]$. Since $d_{i}=d_{i}^{\bullet}$ for all $i$, similarly to above it suffices to check $d_{k} \succ_{\mathcal{P}} e_{k}$ only for $k$ satisfying $k \leq q$ and either $k>q^{\bullet}$ or $e_{k}<e_{k}^{\bullet}$.

II.i. $b_{m} \bullet+1$ is in Case I(a): direct calculation shows that $q=q^{\bullet}+1$ and $P T_{2}=\left(a_{m}\right)+P T_{2}^{\bullet}$. First we have $p \geq \varrho+1 \geq q^{\bullet}+1=q$ since $d_{\varrho+1}>a_{m} \bullet+1=b_{m^{\bullet}+1} \rightarrow_{\mathcal{P}} e_{q^{\bullet}}$. It remains to check $d_{q} t_{\mathcal{P}} e_{q}$. If $\varrho+1=q$, then $d_{\varrho+1} \rightarrow \rightarrow \mathcal{P} a_{m}=e_{q}$, and thus the result holds. Otherwise, i.e. if $\varrho+1>q$, then $d_{q} \leq d_{\varrho}<a_{m}=e_{q}$, and thus again the result holds.

II.ii. $b_{m+1}$ is in Case I(b): Note that $\varrho+1 \geq r$ since $d_{\varrho+1}^{\bullet}>a_{m} \bullet+1=b_{m} \bullet+1 \rightarrow_{\mathcal{P}} e_{r-1}^{\bullet}$. First assume that $r=q$ or $e_{r+1}^{\bullet} \rightarrow_{\mathcal{P}} b_{m}=a_{m}$. (This includes the case $m=m^{\bullet}+1$.) Then we have $q=q^{\bullet}, e_{r}=a_{m}$, and $e_{k}=e_{k}^{\bullet}$ for $k \neq r$, and thus it suffices to show that $d_{r} \nrightarrow_{\mathcal{P}} e_{r}=a_{m}$. However, if $d_{r} \rightarrow_{\mathcal{P}} a_{m}$ then $d_{\varrho+1} \geq d_{r} \rightarrow_{\mathcal{P}} a_{m}$ thus $d_{\varrho+1} \rightarrow_{\mathcal{P}} a_{m}$, which contradicts the assumption.

From now on we suppose that $e_{r+1}^{\bullet} \not_{\mathcal{P}} a_{m}$ (so that $m \geq m^{\bullet}+2$ ). In this case $a_{m}>e_{r}^{\bullet}$ by Condition (†) since $e_{r+1}^{\bullet} t_{\mathcal{P}} a_{m}$ and $e_{r+1}^{\bullet} \rightarrow_{\mathcal{P}} e_{r}^{\bullet}$. First we assume $m=m^{\bullet}+2$. Then we have 
$a_{m} \leftarrow-\mathcal{P} e_{r+1}^{\bullet}$; otherwise we should have $a_{m}>e_{r+1}^{\bullet}$, but it is impossible by Condition ( $\left.\pitchfork\right)$ since $e_{r+1}^{\bullet} \rightarrow_{\mathcal{P}} e_{r}^{\bullet}>a_{m-1}$ but $a_{m}-\rightarrow \mathcal{P} a_{m-1}$. Now direct calculation shows that $q=q^{\bullet}, e_{r}=a_{m-1}$, and $e_{k}=e_{k}^{\bullet}$ for $k \neq r$, and thus it suffices to show that $d_{r} \nrightarrow_{\mathcal{P}} e_{r}=a_{m-1}$. If $\varrho+1>r$, then we have $d_{r} \leq d_{\varrho}<a_{m-1}=e_{r}$, and thus we are done. Now we claim that $\varrho+1 \neq r$; first note that $d_{\varrho}>e_{r-1}$ since $a_{m-1} \rightarrow_{\mathcal{P}} e_{r-1}^{\bullet}=e_{r-1}$ and $a_{m-1}-\rightarrow_{\mathcal{P}} d_{\varrho}$. Together with $d_{\varrho}=d_{\varrho} \not_{\mathcal{P}} e_{\varrho}^{\bullet}=e_{r-1}$, this means that $d_{\varrho} \rightarrow{ }_{\mathcal{P}} e_{r-1}$. Also $e_{r+1} \rightarrow_{\mathcal{P}} a_{m-1}$ since $e_{r+1}=e_{r+1}^{\bullet} \rightarrow_{\mathcal{P}} e_{r}^{\bullet}>a_{m-1}$. But this means that $\left\{e_{r-1}, d_{\varrho}, a_{m-1}, a_{m}, e_{r+1}\right\}$ is a ladder in $\mathcal{P}$ which $e_{r}^{\bullet}$ is climbing $\left(e_{r+1} \rightarrow_{\mathcal{P}} e_{r}^{\bullet} \rightarrow_{\mathcal{P}} e_{r-1}\right)$, which is a contradiction. (See Figure 41,)

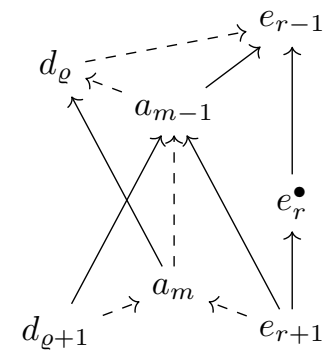

Figure 41. $a_{m}$ in Case II(a), $b_{m}$ in Case $\mathrm{I}(\mathrm{b}), m=m^{\bullet}+2, \varrho+1=r$

It remains to assume that $m \geq m^{\bullet}+3$. We claim that $e_{r}^{\bullet} \not \rightarrow_{\mathcal{P}} a_{m} \bullet+1$. Otherwise, $a_{m-1}<e_{r}^{\bullet}$ by Lemma 9.2 which in turn implies that $a_{m-1} \leftarrow_{\mathcal{P}} e_{r+1}^{\bullet}$ and $a_{m}{ }^{--} \mathcal{P} e_{r+1}^{\bullet}$ by assumption. However, it means that $\left\{a_{m} \bullet+1, \ldots, a_{m}, e_{r+1}^{\bullet}\right\}$ is a ladder that $e_{r}^{\bullet}$ is climbing, which is a contradiction. Since $e_{r}^{\bullet}>a_{m+1}$ by assumption, it follows that $e_{r}^{\bullet-\rightarrow \mathcal{P}} a_{m} \bullet+1$. Now we apply Lemma 9.4 to $\mathcal{L}_{a d}$ (of length $m-m^{\bullet}+2 \geq 5$ ) and $e_{r}^{\bullet}$ and conclude that $d_{\varrho} \leftarrow-\mathcal{P} e_{r}^{\bullet}$ and $a_{m+2}{ }^{--\rightarrow \mathcal{P}} e_{r}^{\bullet}$.

Note that $e_{r+1}^{\bullet} \rightarrow_{\mathcal{P}} a_{m} \bullet+1$ by Condition $(\pitchfork)$ as $e_{r}^{\bullet} \leftarrow_{\mathcal{P}} e_{r+1}^{\bullet}$ and $e_{r}^{\bullet}>a_{m+1}$. Thus by Lemma 9.2 we have $e_{r}^{\bullet} \rightarrow \mathcal{P} a_{j}$ for $j \in\left[m^{\bullet}+1, m-2\right]$ and we may apply Lemma 9.3 to $\left\{a_{m-1}, a_{m}\right\}$ and $e_{r+1}^{\bullet}$. As $a_{m} \nvdash_{\mathcal{P}} e_{r+1}^{\bullet}$ by assumption, case (9.3,4) is excluded. In other cases, direct calculations shows that $e_{r}=a_{m \cdot 1}, e_{k}=e_{k}^{\bullet}$ if $k \notin\{r, r+1\}$, and

○ (9.3. 1): either $e_{r+1}=e_{r+1}^{\bullet}$ or $e_{r+1}=a_{m}>e_{r+1}^{\bullet}$.

○ (9.3.2): $e_{r+1}=a_{m}$.

○ (9.3.3): $e_{r+1}=e_{r+1}^{\bullet}$.

(See Figure 42,) In any case we have $q=q^{\bullet}$. Thus it suffices to check that $d_{r} \succ_{\mathcal{P}} e_{r}$, and $d_{r+1} \neg_{\mathcal{P}} e_{r+1}$ in case $(9.3,2)$. Note that here we have $\varrho+1>r$ since $d_{\varrho+1} \rightarrow_{\mathcal{P}} a_{m-1} \geq a_{m} \bullet+2>e_{r}^{\bullet}$. If $d_{r} \rightarrow_{\mathcal{P}} e_{r}=a_{m} \bullet+1$, then since $d_{r} \leftarrow_{\mathcal{P}} d_{\varrho+1}$ it means that $d_{r}$ is climbing $\mathcal{L}_{a d}$, which is a contradiction. Also in case (9.32) we have $d_{r+1} \nrightarrow_{\mathcal{P}} e_{r+1}=a_{m}$ by Condition ( $\left.\pitchfork\right)$ since $d_{r+1} \leq d_{\varrho+1}$ and $d_{\varrho+1} \rightarrow \mathcal{P} a_{m}$.

II.iii. $b_{m} \bullet+1$ is in Case $\operatorname{II}(\mathrm{a}), u(r+s-1)=m^{\bullet}$ : when $m=m^{\bullet}+1$, then $P T_{2}=P T_{2}^{\bullet}$, and thus there is nothing to check. Thus suppose that $m \geq m^{\bullet}+2$. Note that $e_{r+s}^{\bullet}{ }^{--} \mathcal{P} a_{m+1}=b_{m} \bullet+1$ since $b_{m \bullet+1}$ is in Case II(a) and $u(r+s-1)+1=m^{\bullet}$, and $\varrho \geq r+s$ since $d_{\varrho+1}^{\bullet} \rightarrow \mathcal{P} a_{m} \bullet+1>e_{r+s}^{\bullet}$. First assume that $e_{r+s}^{\bullet} \leftarrow \mathcal{P} a_{m} \bullet+2$. Then $\mathcal{L}_{a d} \cup\left\{e_{r+s}^{\bullet}\right\}-\left\{d_{\varrho}\right\}$ is a ladder and $e_{r+s}^{\bullet} \leftarrow \mathcal{P} e_{r+s+1}^{\bullet}$, and thus by Lemma 9.2 we have $e_{r+s}^{\bullet} \rightarrow \mathcal{P} a_{j}$ for $j \in\left[m^{\bullet}+1, m-2\right]$ and we may apply Lemma 9.3 to $\left\{a_{m-1}, a_{m}\right\}$ and $e_{r+s+1}^{\bullet}$ (9.3) is impossible since $b_{m+1}$ is in Case II(a), i.e. there exists 


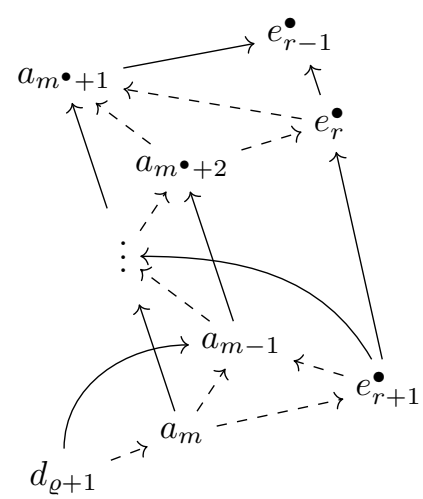

$9.31)$

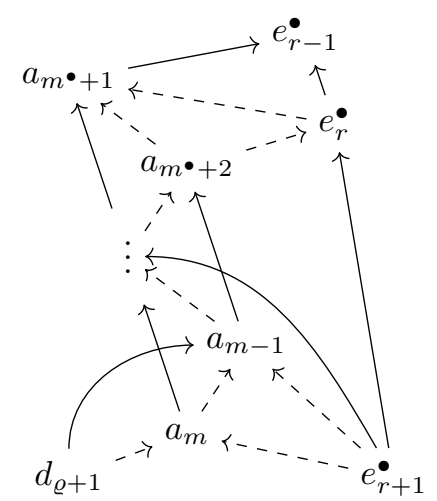

$(9.32)$

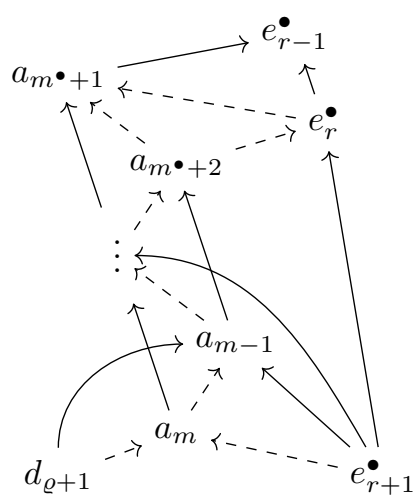

$9.3 .3)$

Figure 42. $a_{m}$ in Case II(a), $b_{m}$ in Case $\mathrm{I}(\mathrm{b}), m \geq m^{\bullet}+3$

$j \in\left[m^{\bullet}+1, m\right]$ such that $a_{j}=b_{j}{ }^{--\mathcal{P}} e_{r+s+1}^{\bullet}$. In other cases, direct calculation shows that $e_{k}=e_{k}^{\bullet}$ if $k \neq r+s+1$ and

○ (9.3,1): either $e_{r+s+1}=e_{r+s+1}^{\bullet}$ or $e_{r+s+1}=a_{m}>e_{r+s+1}^{\bullet}$.

○ (9.32): $e_{r+s+1}=a_{m}$.

○ (9.3.3): $e_{r+s+1}=e_{r+s+1}^{\bullet}$.

(See Figure 43.) In any case we have $q=q^{\bullet}$. Thus we only need to check that $d_{r+s+1} \nrightarrow_{\mathcal{P}} e_{r+s+1}=$ $a_{m}$ in case $(9.32)$. But this holds by Condition $(\pitchfork)$ since $d_{r+s+1} \leq d_{\varrho+1}$ and $d_{\varrho+1}{ }_{--\mathcal{P}} a_{m}=$ $e_{r+s+1}$.

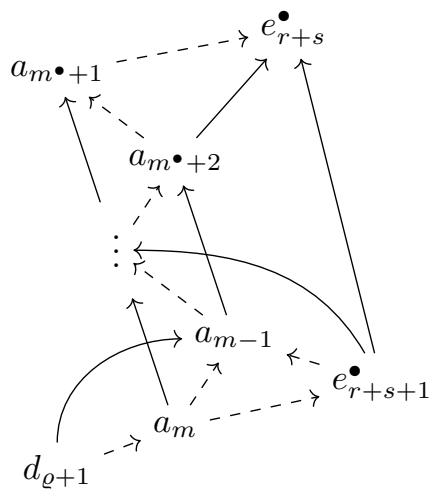

$9.31)$

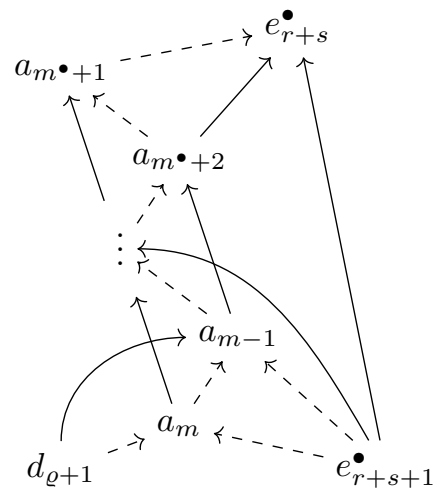

$9.32)$

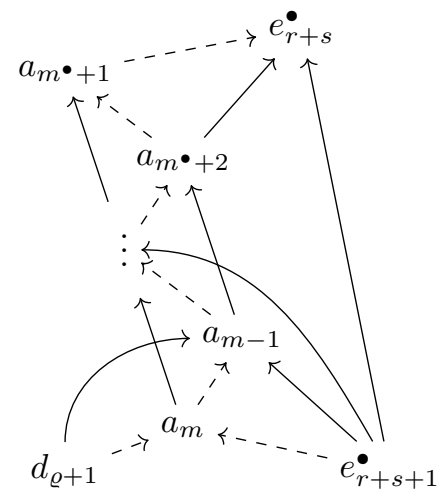

$9.33)$

Figure 43. $a_{m}$ in Case II(a), $b_{m}$ in Case $\mathrm{II}(\mathrm{a}), u(r+s-1)=m^{\bullet}$, $m \geq m^{\bullet}+2, e_{r+s}^{\bullet} \leftarrow \mathcal{P} a_{m} \bullet+2$

It remains to assume that $e_{r+s}^{\bullet}{ }^{--\mathcal{P}} a_{m+2}$. Since $e_{r+s}^{\bullet} \leftarrow \mathcal{P} e_{r+s+1}^{\bullet}$, this means that $a_{m} \bullet+2<$

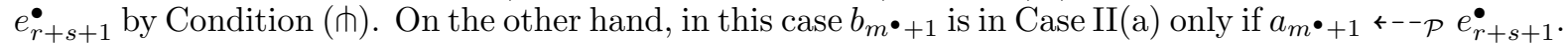


Thus we may apply Lemma 9.4 to $\mathcal{L}_{a d}$ and $e_{r+s+1}^{\bullet}$. Here (9.4.3) is the only one satisfying $a_{m+2}<e_{r+s+1}^{\bullet}$, in which case $m=m^{\bullet}+2$ and $P T_{2}=P T_{2}^{\bullet}$. (See Figure 44) Thus there is nothing to prove.

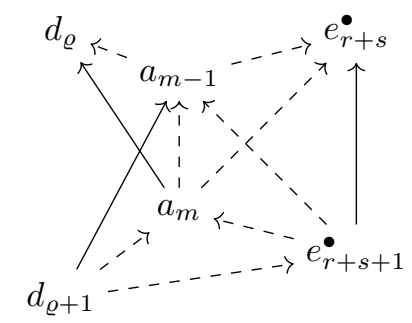

Figure 44. $a_{m}$ in Case II(a), $b_{m}$ in Case II(a), $u(r+s-1)=m^{\bullet}, m \geq m^{\bullet}+2, e_{r+s}^{\bullet} \leftarrow-\mathcal{P} a_{m} \bullet+2$

II.iv. $b_{m \bullet+1}$ is in Case II(a), $u(r+s-1)<m^{\bullet}$ : first assume that $m=m^{\bullet}+1$. Then $q=q^{\bullet}$, $e_{r+s}^{\bullet}=b_{m-1}, e_{r+j}^{\bullet}=b_{u(r+j)}$ for $j \in[1, s-1]$, and $e_{j}=e_{j}^{\bullet}$ if $j \notin[r+1, r+s]$. Thus it suffices to check $d_{k} t_{\mathcal{P}} e_{k}$ for $k \in[r+1, r+s]$. Since $d_{\varrho+1}^{\bullet}=d_{\varrho+1}>a_{m}=b_{m}>b_{m-1}=e_{r+s}^{\bullet}$, we have $r+s \leq \varrho+1$. Also since $d_{\varrho+1} \rightarrow \mathcal{P} a_{m}=b_{m}$, by Lemma 9.1 either $\mathcal{L}_{b e} \cup\left\{d_{\varrho+1}\right\}$ or $\mathcal{L}_{b e} \cup\left\{d_{\varrho+1}\right\}-\left\{b_{m}\right\}$ is a ladder. Now if $d_{k} \rightarrow_{\mathcal{P}} e_{k}$ for some $k \in[r+1, r+s], k<\varrho+1$ then as $d_{k} \leftarrow_{\mathcal{P}} d_{\varrho+1}$ it implies that $d_{k}$ is climbing a ladder, which is impossible. It remains to check the case when $k=r+s=\varrho+1$. We claim that this is impossible. First, $\varrho+2=r+s+1 \geq q \geq p$, and thus $d_{\varrho+2}$ is well-defined. Furthermore, $d_{\varrho+2}=d_{\varrho+2}^{\bullet} t_{\mathcal{P}} e_{r+s+1}^{\bullet}=e_{r+s+1}$, and thus $d_{\varrho+1}<e_{r+s+1}$ by Condition ( $\pitchfork$ ) since $d_{\varrho+2} \rightarrow_{\mathcal{P}} d_{\varrho+1}$. On the other hand, if $d_{\varrho+2}<e_{r+s+1}$ then $a_{m}<d_{\varrho+1} \leftarrow \mathcal{P} d_{\varrho+2}<e_{r+s+1}$ which implies $b_{m}=a_{m} \leftarrow \mathcal{P} e_{r+s+1}$, but it contradicts the assumption that $b_{m}$ is in Case II(a). Therefore, we have $d_{\varrho+1}<e_{r+s+1}<d_{\varrho+2}$ and also $d_{\varrho+2} \rightarrow{ }^{-\rightarrow \mathcal{P}} e_{r+s+1}$. Now one may check that $\mathcal{P}$ restricted to $\left\{d_{\varrho}, a_{m}, d_{\varrho+1}, e_{r+s+1}, d_{\varrho+2}\right\}$ is isomorphic to $\mathcal{P}_{(3,1,1), 5}$, which is a contradiction. (See Figure 45)

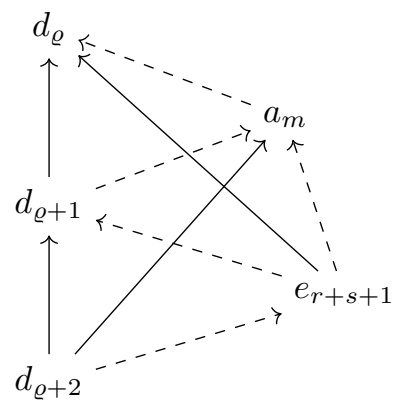

Figure 45. $a_{m}$ in Case II(a), $b_{m}$ in Case II(a), $m=m^{\bullet}+1, \varrho+1=r+s$

Now assume that $m \geq m^{\bullet}+2$. First $d_{\varrho+1}^{\bullet}=d_{\varrho+1} \rightarrow_{\mathcal{P}} a_{m} \bullet+1>b_{m} \bullet=e_{r+s}^{\bullet}$, and thus $\varrho \geq r+s$. As $\mathcal{L}_{b e}$ is a ladder of size $\geq 3$ by assumption, $\mathcal{L}_{b e} \cup\left(\mathcal{L}_{a d}-\left\{d_{\varrho}\right\}\right)$ is also a ladder by Lemma 3.11. Now by Lemma 9.4 we have $e_{r+s+1}^{\bullet} \rightarrow \mathcal{P} x$ for any $x \in \mathcal{L}_{b e} \cup\left(\mathcal{L}_{a d}-\left\{d_{\varrho}\right\}\right)-\left\{a_{m-1}, a_{m}, d_{\varrho+1}\right\}$, and we may apply 9.3 to $\left\{a_{m-1}, a_{m}\right\}$ and $e_{r+s+1}^{\bullet}$. Keep in mind that $b_{m+1}$ is in Case II(a) which 
implies that (9.34) is impossible. Direct calculation shows that $e_{r+j}^{\bullet}=b_{u(r+j)}$ for $j \in[1, s-1]$, $e_{r+s}^{\bullet}=b_{m} \cdot, e_{k}=e_{k}^{\bullet}$ if $k \notin[r+1, r+s+1\}$ and

○ (9.3): either $e_{r+s+1}=e_{r+s+1}^{\bullet}$ or $e_{r+s+1}=a_{m}>e_{r+s+1}^{\bullet}$.

$\circ$ (9.3,2): $e_{r+s+1}=a_{m}$.

$\circ 9.3,3): e_{r+s+1}=e_{r+s+1}^{\bullet}$.

(See Figure 46]) In any case we have $q=q^{\bullet}$. Thus it suffices to show that $d_{k} t_{\mathcal{P}} e_{k}$ if $k \in[r+1, r+s]$ and $d_{r+s+1} t_{\mathcal{P}} e_{r+s+1}$ in case $(9.32)$. The former is clear. Indeed, if $k \in[r+1, r+s]$ then $k<\varrho+1$, and thus $d_{k} \leftarrow_{\mathcal{P}} d_{\varrho+1}$. Therefore, if $d_{k} \rightarrow_{\mathcal{P}} e_{k}$ then $d_{k}$ is climbing $\mathcal{L}_{b e} \cup\left(\mathcal{L}_{a d}-\left\{d_{\varrho}\right\}\right)$ which is a contradiction. Now if we are in case $(9.32)$ then $d_{\varrho+1} \geq d_{r+s+1}$ and $d_{\varrho+1} \rightarrow_{\rightarrow \mathcal{P}} a_{m}=e_{r+s+1}$, and thus $d_{r+s+1} \nrightarrow_{\mathcal{P}} e_{r+s+1}$.

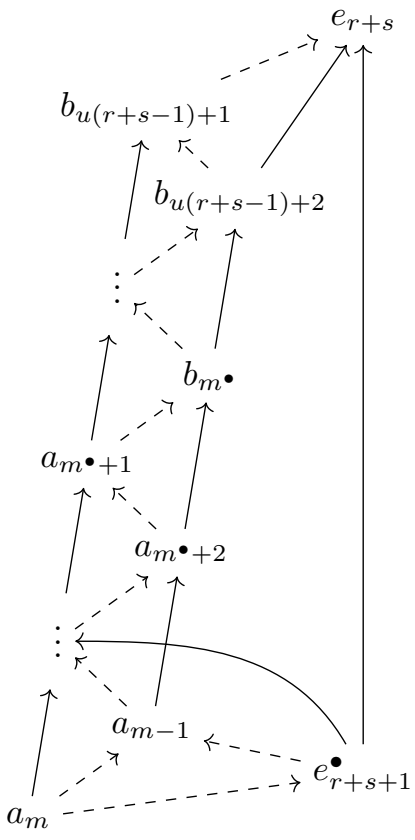

(9.3. 1)

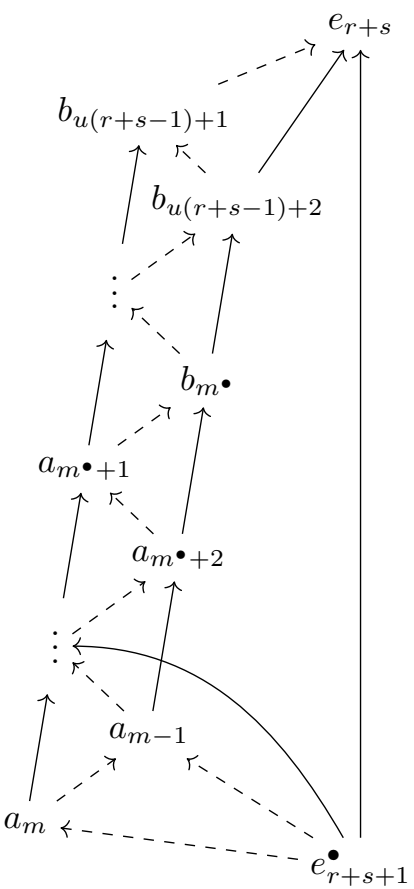

(9.3)

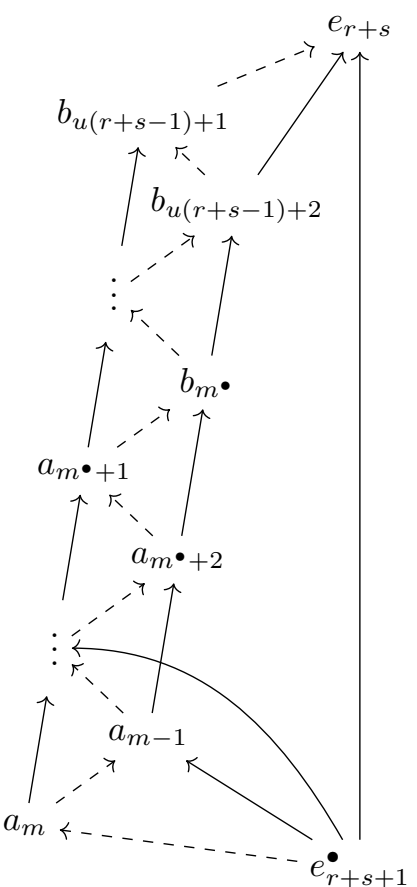

$9.33)$

Figure 46. $a_{m}$ in Case II(a), $b_{m}$ in Case II(a), $u(r+s-1)<m^{\bullet}, m \geq m^{\bullet}+2$

II.v. $b_{m} \bullet+1$ is in Case II(b), $u(r+s-1)<m^{\bullet}:$ note that $\mathcal{L}_{b e} \ni a_{m} \bullet+1, b_{m} \bullet, e_{r+s}^{\circ}$, and thus $\mathcal{L}_{b e}$ is of length $\geq 3$. If $m>m^{\bullet}+1$ then $\mathcal{L}_{b e} \cup\left(\mathcal{L}_{a d}-\left\{d_{\varrho}\right\}\right)$ is a ladder by Lemma 3.11, and thus so is $\mathcal{L}_{b e} \cup\left(\mathcal{L}_{a d}-\left\{d_{\varrho}, d_{\varrho+1}\right\}\right)$. If $m=m^{\bullet}+1$ then $\mathcal{L}_{b e} \cup\left(\mathcal{L}_{a d}-\left\{d_{\varrho}, d_{\varrho+1}\right\}\right)=\mathcal{L}_{b e}$ is clearly a ladder. Then direct calculation shows that $q=q^{\bullet}, e_{r+s}=b_{m}>b_{m-1}=e_{r+s}^{\bullet}$, and $e_{i}=e_{i}^{\bullet}$ otherwise, and thus there is nothing to check here.

II.vi. $b_{m} \bullet+1$ is in Case $\mathrm{II}(\mathrm{b}), u(r+s-1)=m^{\bullet}$ : first assume that $\mathcal{L}_{b e} \cup\left(\mathcal{L}_{a d}-\left\{d_{\varrho}, d_{\varrho+1}\right\}\right)=$ $\mathcal{L}_{b e} \sqcup\left\{a_{m} \bullet+2, \ldots, a_{m}\right\}$ is a ladder. Then direct calculation shows that $e_{r+i}=b_{u(r+i)}>e_{r+i}^{\bullet}$ for $i \in[1, s-1], e_{r+s}=b_{m}>e_{r+s}^{\bullet}$, and $e_{i}=e_{i}^{\bullet}$ if $i \notin[r+1, r+s]$, and thus there is nothing to check. 
Thus suppose otherwise, i.e. $\mathcal{L}_{b e} \cup\left\{a_{m} \bullet+2, \ldots, a_{m}\right\}$ is not a ladder and in particular $m \geq m^{\bullet}+2$. Since $\left\{a_{m} \bullet+1, \ldots, a_{m}, d_{\varrho+1}\right\}$ is a ladder of length $\geq 3$, by Lemma 3.11 the length of $\mathcal{L}_{b e}$ should be at most 2, i.e. $\mathcal{L}_{b e}=\left\{e_{r+1}^{\bullet}, a_{m+1}\right\}$ and also $e_{r+1}^{\bullet}{ }^{\bullet-} \mathcal{p} a_{m} \bullet+2$. Also we have either $q^{\bullet}=r+1$ or $e_{r+2}^{\bullet} \rightarrow \mathcal{P} a_{m} \bullet_{+1}=b_{m} \bullet+1$ since $b_{m} \bullet+1$ is in Case II(b). If $q^{\bullet}>r+1$ then we may apply Lemma 9.3 to $\left\{a_{m-1}, a_{m}\right\}$ and $e_{r+2}^{\bullet}$. Again since $b_{m} \bullet_{+1}$ is in Case II(b), the only possible cases is $(9.3,4)$, i.e. $a_{m} \leftarrow_{\mathcal{P}} e_{r+2}^{\bullet}$. Now direct calculation shows that $q=q^{\bullet}, e_{r+1}=a_{m}>e_{r+1}^{\bullet}$ and $e_{i}=e_{i}^{\bullet}$ otherwise. Thus there is nothing to check. (See Figure 47.)

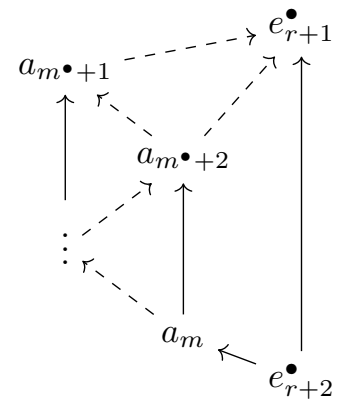

Figure 47. $a_{m}$ in Case II(a), $b_{m}$ in Case II(b), $u(r+s-1)=m^{\bullet}$, $\mathcal{L}_{b e} \sqcup\left\{a_{m} \bullet+2, \ldots, a_{m}\right\}$ is not a ladder

III. $a_{m}$ is in Case II(b). Recall that $b_{i}=a_{i}$ for $i \in\left[m^{\bullet}+1, m\right]$. Here, we have $d_{i}=d_{i}^{\bullet}$ if

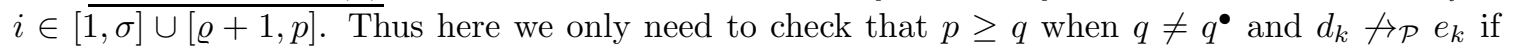
$k \leq q$ and either $k>q^{\bullet}, e_{k}<e_{k}^{\bullet}$, or $k \in[\sigma+1, \varrho]$.

III.i. $b_{m} \bullet+1$ is in Case I(a): direct calculation shows that $q=q^{\bullet}+m-m^{\bullet}$ and $P T_{2}=\left(d_{\varrho}^{\bullet}, \ldots, d_{\sigma+1}^{\bullet}\right)+$ $P T_{2}^{\bullet}$. Note that $\sigma \geq q^{\bullet}$ since $d_{\sigma+1}^{\bullet}=b_{m} \bullet+1 \rightarrow_{\mathcal{P}} e_{q} \bullet$, and thus in particular $q \leq \sigma+m-m^{\bullet}=\varrho \leq p$. It remains to check $d_{k} t_{\mathcal{P}} e_{k}$ for $k \in\left[q^{\bullet}+1, q\right]$. However, since $e_{q+i}=b_{m} \bullet+i=d_{\sigma+i}^{\bullet}{ }^{\bullet-\mathcal{P}} a_{m+i}=$ $d_{\sigma+i}$ and $d_{\sigma+i} \geq d_{q^{\bullet+i}}$ for $i \in\left[1, q-q^{\bullet}\right]$, we have $d_{q^{\bullet}+i} \dashv_{\mathcal{P}} e_{q} \bullet^{\bullet+i}$ by Condition ( $\pitchfork$ ) as desired.

III.ii. $b_{m} \bullet+1$ is in Case I(b): we claim that

$-q=\max \left\{q^{\bullet}, r+m-m^{\bullet}-1\right\}$,

$-e_{r+i}=b_{m} \bullet+i+1=d_{\sigma+i+1}^{\bullet}$ for $i \in\left[0, m-m^{\bullet}-1\right]$ and $e_{k}=e_{k}^{\bullet}$ otherwise, and

$-b_{m} \bullet+i+1$ are in Case I(either (a) or (b)) if $i<m-m^{\bullet}-1$.

(See Figure 48,) Note that the first part follows from the other parts. To this end, we use induction on $i$. If $i=0$ then it is obvious by assumption. Now assume that the result is true up to $i-1$. First

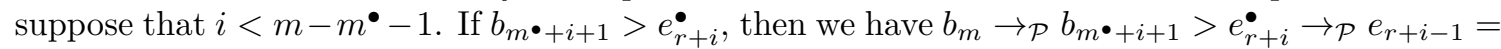
$b_{m} \bullet+i$ which means that $e_{r+i}^{\bullet}$ is climbing $\mathcal{L}_{a d}$, a contradiction. Thus $b_{m} \bullet+i+1<e_{r+i}^{\bullet}$, and direct calculation shows that $b_{m} \bullet+i+1$ is in Case I and $e_{r+i}=b_{m} \bullet+i+1$.

Finally, we assume $i=m-m^{\bullet}-1>0$. If $q^{\bullet}<r+m-m^{\bullet}-1$ or $b_{m}<e_{r+m-m}^{\bullet} \bullet_{-1}$ then $b_{m}$ is in Case I and the result is obvious, and thus suppose otherwise. If $e_{r+m-m}^{\bullet} \bullet_{-1} \leftarrow \mathcal{P} b_{m}$, then $b_{m} \rightarrow_{\mathcal{P}} e_{r+m-m}^{\bullet} \bullet_{-1} \rightarrow_{\mathcal{P}} b_{m-1}, a_{m-1} \leftrightarrow_{-\mathcal{P}} b_{m}$, and $a_{m-1}-_{-\rightarrow \mathcal{P}} b_{m-1}$, which contradicts Lemma 3.4(1). Thus we have $e_{r+m-m}^{\bullet} \bullet_{-1} \leftarrow^{-\mathcal{P}} b_{m}$. If $q^{\bullet}<r+m-m$ or $b_{m} \leftarrow_{\mathcal{P}} e_{r+m-m}^{\bullet}$, then $b_{m}$ is in Case $\mathrm{II}(\mathrm{b})$ and $e_{r+m-m^{\bullet}} \boldsymbol{\bullet}_{-1}=b_{m}$, and thus we are done. It remains to consider the case when $q^{\bullet} \geq r+m-m^{\bullet}$ and $e_{r+m-m}^{\bullet} \boldsymbol{\bullet}^{\bullet} \rightarrow_{\mathcal{P}} b_{m}$, so that $b_{m}$ is in Case II(a). But then 
$a_{m-1} \leftarrow_{\mathcal{P}} e_{r+m-m}^{\bullet} \bullet$ by Lemma 3.4(1) applied to $b_{m-1}, e_{r+m-m}^{\bullet_{-1}}, e_{r+m-m}^{\bullet}$, and $a_{m-1}$. This means that $\left\{b_{m-1}, a_{m-1}, b_{m}, e_{r+m-m}^{\bullet}\right\}$ is a ladder in $\mathcal{P}$ which $e_{r+m-m}^{\bullet_{-1}}$ is climbing, which is a contradiction. (See Figure 49,

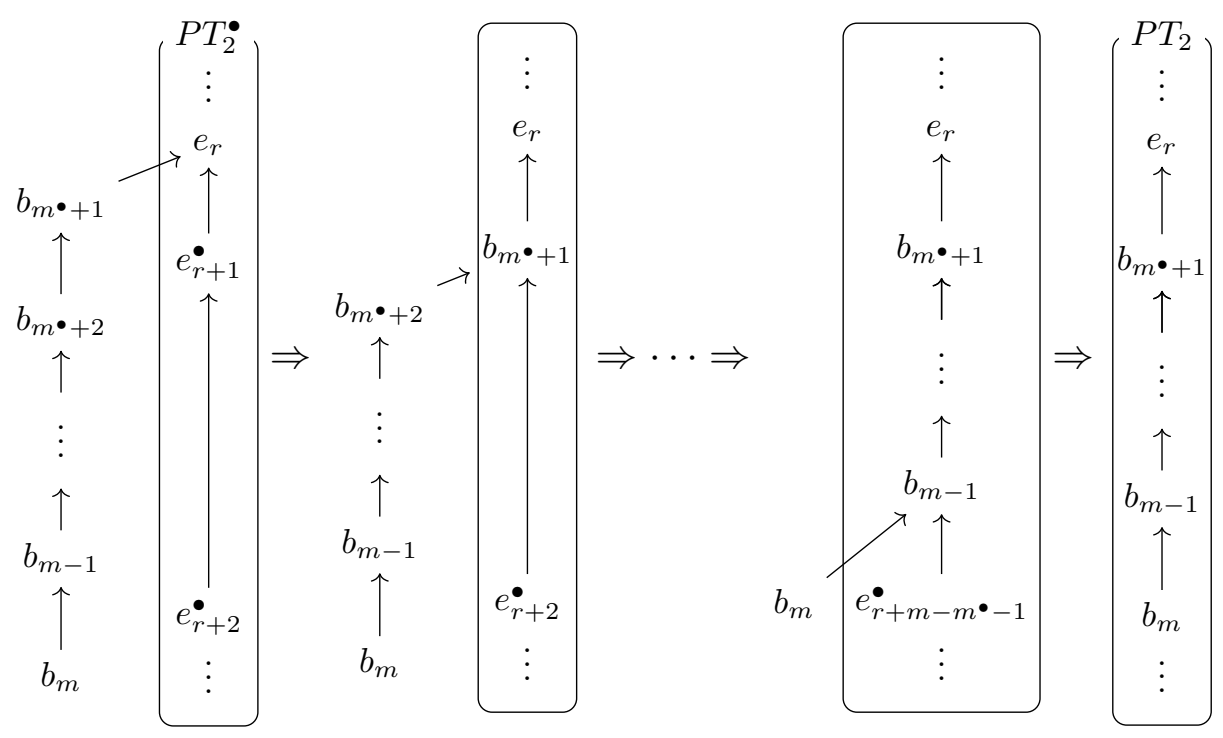

Figure 48. $a_{m}$ in Case II(b), $b_{m} \bullet+1$ in Case I(b)

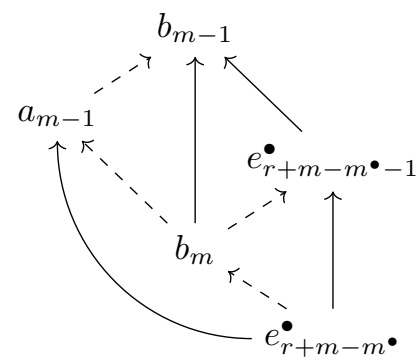

Figure 49. $a_{m}$ in Case II(b), $b_{m} \bullet+1$ in Case I(b),

$$
e_{r+m-m} \bullet-\rightarrow \mathcal{P} b_{m}, b_{m} \rightarrow \mathcal{P} e_{r+m-m} \bullet-1
$$

Note that $\sigma+1 \geq r$ as $d_{\sigma+1}^{\bullet}=b_{m+1} \rightarrow_{\mathcal{P}} e_{r-1}^{\bullet}$. Thus $r+m-m^{\bullet}-1 \leq \sigma+m-m \bullet=\varrho \leq p$, which means that $q \leq p$. It remains to check that $d_{k} \succ_{\mathcal{P}} e_{k}$ for $k \in[\sigma+1, \varrho] \cup\left[r, r+m-m^{\bullet}-1\right]$, $k \leq q$. If $k \in[\sigma+1, \varrho]$ then we have $d_{k}=a_{k+m-\varrho}{ }^{-\rightarrow \mathcal{P}} b_{k+m-\varrho}=e_{r+k+m-\varrho-m} \bullet_{-1}=e_{r+k-\sigma-1}$ and $e_{r+k-\sigma-1} \leq e_{k}$, which means that $d_{k} t_{\mathcal{P}} e_{k}$ by Condition ( $\left.\pitchfork\right)$. Similarly, if $k \in\left[r, r+m-m^{\bullet}-1\right]$ then $d_{k+\sigma+1-r} \rightarrow_{\mathcal{P}} e_{k}$ and $d_{k+\sigma+1-r} \geq d_{k}$, and thus $d_{k} \nrightarrow_{\mathcal{P}} e_{k}$ by Condition ( $\pitchfork$ ).

III.iii. $b_{m} \bullet+1$ is in Case II(a), $u(r+s-1)=m^{\bullet}$ : note that $\sigma+1 \geq r+s$ since $d_{\sigma+1}^{\bullet}=b_{m} \bullet+1>e_{r+s}^{\bullet}$. First we assume that $m=m^{\bullet}+1$, in which case $P T_{2}=P T_{2}^{\bullet}$. Here it suffices to check that $d_{k} \succ_{\mathcal{P}} e_{k}$ for $k=\sigma+1=\varrho, k \leq q$. It is obvious when $\sigma+1=\varrho>r+s$ by Condition 


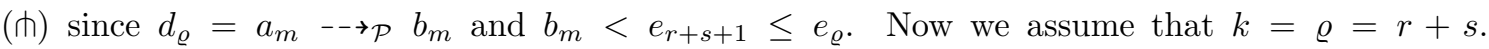
Then $\varrho+1 \leq p$ since $\varrho+1=r+s+1 \leq q \leq p$, and $d_{\varrho+1}>e_{r+s+1}$ by Condition ( $\pitchfork$ ) since $d_{\varrho+1}=d_{\varrho+1}^{\bullet} \rightarrow \mathcal{P} d_{\varrho}^{\bullet}=b_{m}$ and $b_{m}{ }^{--\mathcal{P}} e_{r+s+1}$. As $d_{\varrho}=d_{\varrho+1}^{\bullet} t_{\mathcal{P}} e_{r+s+1}^{\bullet}=e_{r+s+1}$ by assumption, it means that $d_{\varrho+1} \rightarrow{ }_{\varrho} e_{r+s+1}$, i.e. $\left\{e_{r+s}, b_{m}, e_{r+s+1}, d_{\varrho+1}\right\}$ is a ladder. Now if $d_{\varrho} \rightarrow \mathcal{P} e_{r+s}$ then $d_{\varrho}$ is climbing $\left\{e_{r+s}, b_{m}, e_{r+s+1}, d_{\varrho+1}\right\}$, which is a contradiction. (See Figure [50)

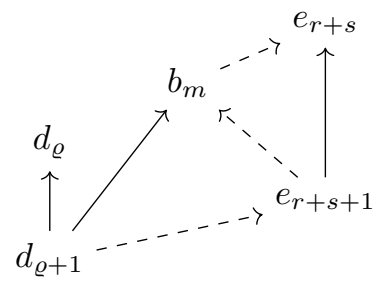

Figure 50. $a_{m}$ in Case II(b), $b_{m} \bullet+1$ in Case II(a),

$$
u(r+s-1)=m^{\bullet}, m=m^{\bullet}+1, \varrho=r+s
$$

Now we show that the case $m>m^{\bullet}+1$ is impossible. For the sake of contradiction we assume this condition. Here $b_{m} \bullet+1$ is in Case II(a) only if $e_{r+s+1}^{\bullet} \rightarrow_{\mathcal{P}} b_{m} \bullet+1$, which in turn

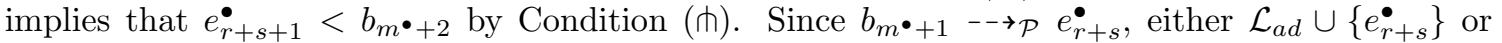
$\mathcal{L}_{a d} \cup\left\{e_{r+s}^{\bullet}\right\}-\left\{b_{m} \bullet+1\right\}$ is a ladder by Lemma 9.1 If $m>m^{\bullet}+2$ or $\mathcal{L}_{a d} \cup\left\{e_{r+s}^{\bullet}\right\}$ is a ladder

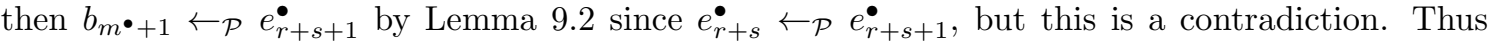
$m=m^{\bullet}+2$ and $\mathcal{L}_{a d} \cup\left\{e_{r+s}^{\bullet}\right\}-\left\{b_{m-1}\right\}$ is a ladder, i.e. $e_{r+s}^{\bullet}{ }^{\bullet-\mathcal{P}} a_{m-1}$. Now we apply Lemma 9.3 to $\left\{a_{m-1}, b_{m}\right\}$ and $e_{r+s+1}^{\bullet}$; the only possible case is (9.3.1) since $e_{r+s+1}^{\bullet}<b_{m}$. However, in this case $b_{m-1}$ is in Case II(b) (together with $b_{m}$ ), which is a contradiction. (See Figure [51,)

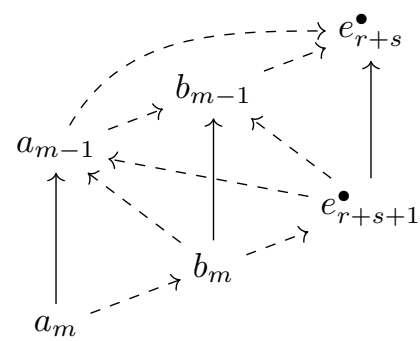

Figure 51. $a_{m}$ in Case II(b), $b_{m} \bullet+1$ in Case II(a),

$$
u(r+s-1)=m^{\bullet}, m>m^{\bullet}+1
$$

III.iv. $b_{m} \bullet+1$ is in Case II(a), $u(r+s-1)<m^{\bullet}$ : first we assume that $m=m^{\bullet}+1$, in which case $q=q^{\bullet}, e_{r+i}^{\bullet}=b_{u(r+i)}$ for $i \in[1, s-1], e_{r+s}^{\bullet}=b_{m-1}$, and $e_{i}=e_{i}^{\bullet}$ otherwise. Thus here it suffices to check that $d_{k} t_{\supset} \mathcal{P} e_{k}$ for $k \in\{\varrho\} \cup[r+1, r+s], k \leq q$. Note that $\varrho \leq r+s$ since $d_{\varrho}^{\bullet}=b_{m}>b_{m-1}=e_{r+s}^{\bullet}$. For $k<\varrho$, first note that either $\mathcal{L}_{b e} \cup\left\{d_{\varrho}\right\}$ or $\mathcal{L}_{b e} \cup\left\{d_{\varrho}\right\}-\left\{b_{m}\right\}$ is a ladder by Lemma 9.1 since $b_{m}{ }^{\leftarrow-\mathcal{P}} a_{m}=d_{\varrho}$. Thus if $d_{k} \rightarrow_{\mathcal{P}} e_{k}$ then $d_{k}$ is climbing a ladder as $d_{k} \leftarrow \mathcal{P} d_{\varrho}$, which is a contradiction. It remains to consider the case $k=\varrho$. If $\varrho<r+s$, then it follows from Condition (内) since $d_{\varrho}=a_{m} \rightarrow_{\mathcal{P}} b_{m}$ and $b_{m}<e_{r+s+1}^{\bullet}=e_{r+s+1} \leq e_{\varrho}$. If $k=\varrho=r+s$, then first $\varrho+1=r+s+1 \leq q \leq p$, and $d_{\varrho+1}=d_{\varrho+1}^{\bullet} \rightarrow \mathcal{P} d_{\varrho}^{\bullet}=b_{m}$. Since $d_{\varrho+1} \nrightarrow_{\mathcal{P}} e_{r+s+1}$ and 
$e_{r+s+1} \rightarrow b_{\mathcal{P}} b_{m}$, by Condition ( $\left.\pitchfork\right)$ it follows that $d_{\varrho+1} \rightarrow \rightarrow_{\mathcal{P}} e_{r+s+1}$, i.e. $\mathcal{L}_{b e} \cup\left\{e_{r+s+1}, d_{\varrho+1}\right\}$ is a ladder in $\mathcal{P}$. Now if $d_{\varrho} \rightarrow_{\mathcal{P}} e_{r+s}$ then as $d_{\varrho} \leftarrow \mathcal{P} d_{\varrho+1}$ it implies that $d_{\varrho}$ is climbing a ladder, which is contradiction. (See Figure 52 .)

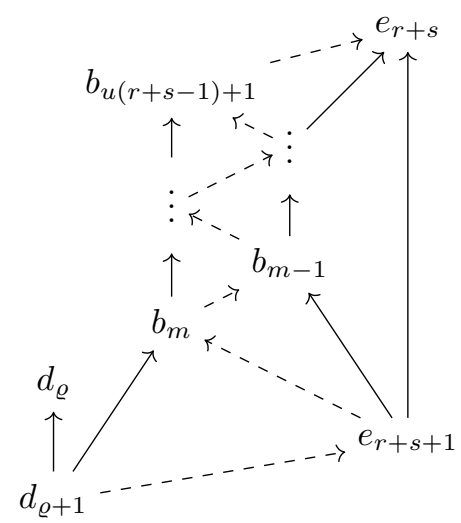

Figure 52. $a_{m}$ in Case II(b), $b_{m}$ in Case II(a), $u(r+s-1)<m^{\bullet}$,

$$
m=m^{\bullet}+1, \varrho=r+s
$$

Now assume that $m>m^{\bullet}+1$, and we prove that this is impossible. Indeed, $b_{m} \bullet+1{ }^{--\mathcal{p}} e_{r+s+1}^{\circ}=$ $e_{r+s+1}^{\bullet}$ since $b_{m} \bullet+1 \leftarrow_{\mathcal{P}} b_{m} \bullet+2$ and $b_{m} \bullet+1$ is in Case II(a). However, since $\mathcal{L}_{b e} \cup \mathcal{L}_{a d}$ is a ladder by Lemma 3.11 and $e_{r+s}=e_{r+s}^{\circ} \leftarrow \mathcal{P} e_{r+s+1}^{\circ}=e_{r+s+1}^{\bullet}$, we should have $b_{m+1} \leftarrow_{\mathcal{P}} e_{r+s+1}^{\bullet}$ by Lemma 9.2. This is a contradiction. (See Figure 53)

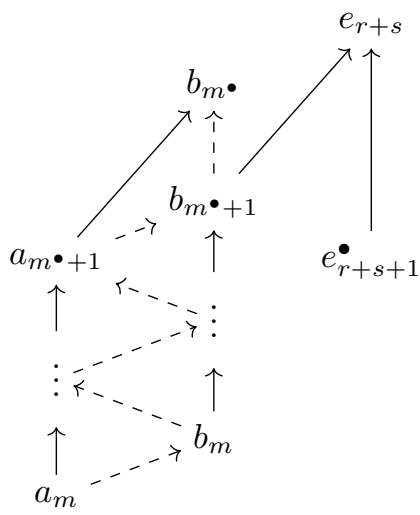

Figure 53. $a_{m}$ in Case II(b), $b_{m} \cdot+1$ in Case II(a),

$$
u(r+s-1)<m^{\bullet}, m>m^{\bullet}+1
$$

III.v. $b_{m} \bullet+1$ is in Case II(b), $u(r+s-1)<m^{\bullet}$ : first we assume that $m=m^{\bullet}+1$, in which case $q=q^{\bullet}, e_{r+s}=b_{m}>b_{m-1}=e_{r+s}^{\bullet}$, and $e_{i}=e_{i}^{\bullet}$ otherwise. Thus here it suffices to check that $d_{k} \neg_{\mathcal{P}} e_{k}$ for $k=\varrho, k \leq q$. Note that $\varrho \geq r+s$ since $d_{\varrho}^{\bullet}=b_{m}>b_{m-1}=e_{r+s}^{\bullet}$, which implies $e_{\varrho} \geq e_{r+s}$. As $d_{\varrho}=a_{m}{ }_{-\rightarrow \mathcal{P}} b_{m}=e_{r+s}$, the result follow from Condition (内). 
Now we assume that $m>m^{\bullet}+1$. Then $\mathcal{L}_{b e} \cup \mathcal{L}_{a d}$ is a ladder by Lemma 3.11 and thus if $q \leq$ $r+s+1$ then $b_{m} \bullet+1 \leftarrow_{\mathcal{P}} e_{r+s+1}^{\circ}=e_{r+s+1}^{\bullet}$ by Lemma 9.2 as $e_{r+s+1}^{\bullet}=e_{r+s+1}^{\circ} \rightarrow_{\mathcal{P}} e_{r+s}^{\circ}$. Now almost the same argument as in III.ii. applies here and one can show that $q=\max \left\{q^{\bullet}, r+s+m-m^{\bullet}-1\right\}$, $e_{r+s+i}=b_{m}^{\bullet+i+1}=d_{\sigma+i+1}^{\bullet}$ for $i \in\left[0, m-m^{\bullet}-1\right]$, and $e_{i}=e_{i}^{\bullet}$ otherwise. First, we have $\sigma+1 \geq r+s$ since $d_{\sigma+1}^{\bullet}=b_{m} \bullet+1>b_{m} \bullet=e_{r+s}^{\bullet}$. Thus $r+s+m-m^{\bullet}-1 \leq \sigma+m-m^{\bullet}=\varrho \leq p$, which means that $q \leq p$. It remains to verify that $d_{k} \not_{\mathcal{P}} e_{k}$ for $k \in[\sigma+1, \varrho] \cup\left[r+s, r+s+m-m^{\bullet}-1\right], k \leq q$. If $k \in[\sigma+1, \varrho]$ then we have $d_{k}=a_{k+m-\varrho^{--\rightarrow \mathcal{P}}} b_{k+m-\varrho}=e_{r+s+k+m-\varrho-m} \bullet-1=e_{r+s+k-\sigma-1}$ and $e_{r+s+k-\sigma-1} \leq e_{k}$, which means that $d_{k} \nrightarrow_{\mathcal{P}} e_{k}$ by Condition (†). Similarly, if $k \in[r+s, r+s+$ $\left.m-m^{\bullet}-1\right]$ then $d_{k+\sigma+1-r-s} \rightarrow_{\mathcal{P}} e_{k}$ and $d_{k+\sigma+1-r-s} \geq d_{k}$, and thus $d_{k} f_{\mathcal{P}} e_{k}$ by Condition ( $\left.\pitchfork\right)$.

III.vi. $b_{m \cdot+1}$ is in Case $\mathrm{II}(\mathrm{b}), u(r+s-1)=m^{\bullet}$ : note that $\sigma+1 \geq r+s$ as $d_{\sigma+1}^{\bullet}=b_{m} \bullet+1>e_{r+s}^{\bullet}$. First suppose that $r+s+1 \leq q^{\bullet}$ and $e_{r+s+1}^{\bullet-\rightarrow \mathcal{P}} b_{m} \bullet+1$. Since $b_{m+1}$ is in Case II(b), this forces that $m>m^{\bullet}+1$ and $e_{r+s+1}^{\bullet}{ }^{\bullet-\mathcal{P}} b_{m} \bullet+2$. On the other hand, by Lemma 9.1 either $\mathcal{L}_{a d} \cup\left\{e_{r+s}^{\bullet}\right\}$ or $\mathcal{L}_{a d} \cup\left\{e_{r+s}^{\bullet}\right\}-\left\{b_{m} \bullet+1\right\}$ is a ladder, thus we may apply Lemma 9.3 to $\left\{a_{m-1}, b_{m}\right\}$ and $e_{r+s+1}^{\bullet}$. The only possible case is when $m=m^{\bullet}+2$ and we are in (9.3.1), i.e. $a_{m-1} \leftarrow_{--\mathcal{P}} e_{r+s+1}^{\bullet}$ and $e_{r+s+1}^{\bullet}{ }^{\bullet--\mathcal{P}} b_{m}$.

Now direct calculation shows that $q=q^{\bullet}, e_{r+i}=b_{u(r+i)}>e_{r+i}^{\bullet}$ for $i \in[1, s-1], e_{r+s}=$ $b_{m-1}>e_{r+s}^{\bullet}, e_{r+s+1}=b_{m}>e_{r+s+1}^{\bullet}$, and $e_{i}=e_{i}^{\bullet}$ otherwise, and thus it suffices to check $d_{k} \nrightarrow_{\mathcal{P}} e_{k}$ for $k=\{\sigma+1=\varrho-1, \varrho\}, k \leq q$. We have $d_{\varrho-1} \neg_{\mathcal{P}} e_{\varrho-1}$ by Condition $(\pitchfork)$ since $d_{\varrho-1}=$ $a_{m-1} \rightarrow \rightarrow \mathcal{P} b_{m-1}=e_{r+s}$ and $e_{\varrho-1} \geq e_{r+s}$ and similarly $d_{\varrho} \dashv_{\mathcal{P}} e_{\varrho}$ since $d_{\varrho}=a_{m}-\rightarrow \mathcal{P} b_{m}=e_{r+s+1}$ and $e_{\varrho} \geq e_{r+s+1}$. (See Figure [54)

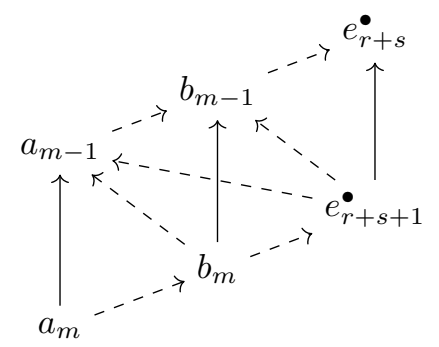

Figure 54. $a_{m}$ in Case $\operatorname{II}(\mathrm{b}), b_{m} \bullet+1$ in Case II(b),

$$
u(r+s-1)=m^{\bullet}, e_{r+s+1}^{\bullet}-\rightarrow \mathcal{P} b_{m}^{\bullet+1}
$$

This time suppose that either $r+s+1>q^{\bullet}$ or $e_{r+s+1}^{\bullet} \rightarrow_{\mathcal{P}} b_{m} \bullet+1$. Then almost the same argument as in III.ii. applies here and one can show that $q=\max \left\{q^{\bullet}, r+s+m-m^{\bullet}-1\right\}$, $e_{r+i}=b_{u(r+i)}>e_{r+i}^{\bullet}$ for $i \in[1, s-1], e_{r+s}=b_{m} \bullet+1>e_{r+s}^{\bullet}, e_{r+s+i}=b_{m} \bullet+i+1=d_{\sigma+i+1}^{\bullet}$ for $i \in\left[1, m-m^{\bullet}-1\right]$, and $e_{i}=e_{i}^{\bullet}$ otherwise. First, we have $\sigma+1 \geq r+s$ since $d_{\sigma+1}^{\bullet}=b_{m} \bullet+1>$ $b_{m} \bullet=e_{r+s}^{\bullet}$. Thus $r+s+m-m^{\bullet}-1 \leq \sigma+m-m^{\bullet}=\varrho \leq p$, which means that $q \leq p$.

It remains to verify that $d_{k} \neg_{\mathcal{P}} e_{k}$ for $k \in[\sigma+1, \varrho] \cup\left[r+s+1, r+s+m-m^{\bullet}-1\right], k \leq q$. If $k \in[\sigma+1, \varrho]$ then we have $d_{k}=a_{k+m-\varrho}{ }_{-\rightarrow \mathcal{P}} b_{k+m-\varrho}=e_{r+s+k+m-\varrho-m-1}=e_{r+s+k-\sigma-1}$ and $e_{r+s+k-\sigma-1} \leq e_{k}$, which means that $d_{k} \dashv_{\mathcal{P}} e_{k}$ by Condition (内). Similarly, if $k \in[r+s+1, r+$ $\left.s+m-m^{\bullet}-1\right]$ then $d_{k+\sigma+1-r-s}{ }_{-\rightarrow \mathcal{P}} e_{k}$ and $d_{k+\sigma+1-r-s} \geq d_{k}$, and thus $d_{k} t_{\mathcal{P}} e_{k}$ by Condition $(\pitchfork)$.

We exhausted all the possibilities and thus proved Theorem 6.1](A) 
9.3. Proof of Theorem 6.1](B), Let us write $w=\left(a_{n}, \ldots, a_{1}\right)$. Then $n-i \in \operatorname{des}_{\mathcal{P}}(w)$ if and only if $a_{i} \leftarrow_{\mathcal{P}} a_{i+1}$. We first show that $a_{i} \leftarrow_{\mathcal{P}} a_{i+1}$ if and only if $i \in \operatorname{des}(Q T)$. The result is trivial if $i$ and $i+1$ are in the same column of $Q T$, and thus suppose otherwise. Then by Proposition [5.5. $i+1$ is in the former column than that of $i$. Since $Q T$ is a standard Young tableau by part (A) it follows that $i$ is in the upper row than $i+1$, and thus $i \in \operatorname{des}(Q T)$. Now suppose that $a_{i} \psi_{\mathcal{P}} a_{i+1}$. By Proposition 5.5, $i$ should be in the former column than that of $i+1$. In this case it is easy to see that $i+1$ cannot be in the lower row than that of $i$ because of the standard Young tableau condition, and thus $i \notin \operatorname{des}(Q T)$.

9.4. Proof of Theorem 6.1] $[\mathrm{C})$, For $w \in \mathfrak{S}_{n}$, let $\mathcal{P}-\mathrm{RS}(w)=\left(P T=\left(P T_{1}, \ldots, P T_{p}\right), Q T\right)$ and choose $w=w_{0}, w_{1}, \ldots, w_{p}=(\infty, \ldots, \infty) \in \mathfrak{A}$ such that $\Phi\left(w_{i}, \emptyset\right)=\left(P T_{i+1}, w_{i+1}\right)$ for $i \in[0, p-1]$. Then by Proposition 5. F(A), we have (recall that $\alpha^{f}$ is the word obtained from $\alpha$ by removing $\infty$ )

$$
w \sim_{\mathcal{P}} P T_{1}+w_{1}^{f} \sim_{\mathcal{P}} P T_{1}+P T_{2}+w_{2}^{f} \sim_{\mathcal{P}} \cdots \sim_{\mathcal{P}} P T_{1}+P T_{2}+\cdots P T_{p}=\operatorname{read}(P T)
$$

as desired.

9.5. Proof of Theorem 6.1](D), By Theorem 6.1] $\left[\right.$ if $\mathcal{P}-\mathrm{RS}(w)=(P T, Q T)$ then $w \sim_{\mathcal{P}} \operatorname{read}(P T)$. Thus by Proposition 4.10 we have g-ht $\operatorname{pos}_{\mathcal{P}}(w)=\mathrm{g}-\mathrm{ht}_{\mathcal{P}}(\operatorname{read}(P T))$. Furthermore, if $w \sim_{\mathcal{P}} w^{\prime}$ and $\mathcal{P}$-RS $\left(w^{\prime}\right)=\left(P T^{\prime}, Q T^{\prime}\right)$ then by the same reason $\mathrm{g}^{-h t_{\mathcal{P}}}(\operatorname{read}(P T)) \sim_{\mathcal{P}} \mathrm{g}^{-h t_{\mathcal{P}}}(w) \sim_{\mathcal{P}} \mathrm{g}-\mathrm{ht}_{\mathcal{P}}\left(w^{\prime}\right) \sim_{\mathcal{P}}$ $\mathrm{g}-\mathrm{ht}_{\mathcal{P}}\left(\operatorname{read}\left(P T^{\prime}\right)\right)$.

Thus it suffices to show that $g$-ht ${ }_{\mathcal{P}}(\operatorname{read}(T))$ equals the length of the first column of $T$ for any $T \in \mathcal{P}$-Tab. Let us denote by $l$ the length of the first column of $T$. Since the first column of $T$ is a subword of genuine $\mathcal{P}$-inversions in $\operatorname{read}(T)$, it follows that g-ht ${ }_{\mathcal{P}}(\operatorname{read}(T)) \geq l$. Now for the sake of contradiction suppose $\mathrm{g}-\mathrm{ht}_{\mathcal{P}}(\operatorname{read}(T))>l$. Then by pigeonhole principle there exists $a, b \in[1, n]$ such that $(a, b) \in{\mathrm{g}-h t_{\mathcal{P}}}(\operatorname{read}(T))$, the column containing $a$ is on the left of that of $b$, and $b$ is not in the upper row than $a$.

Let $c$ be the element located in the intersection of the row of $a$ and the column of $b$. We claim that $(a, c) \in \mathrm{g}^{-h} t_{\mathcal{P}}(\operatorname{read}(T))$. Indeed, if $b=c$ then we are done, and thus suppose otherwise. As $b$ and $c$ are in the same column we have $b \rightarrow_{\mathcal{P}} c$. Since $a \rightarrow_{\mathcal{P}} b$ by assumption, if $(a, c) \notin \mathrm{g}^{-h} t_{\mathcal{P}}(\operatorname{read}(T))$ then by Lemma 8.1 there exists a subword $a e_{1} \cdots e_{k} c$ in $\operatorname{read}(T)$ such that $a \rightarrow \mathcal{P} e_{1} \rightarrow \rightarrow_{\mathcal{P}} \cdots \rightarrow \rightarrow_{\mathcal{P}} e_{k}-\rightarrow \mathcal{P} c$ and $\left\{a, e_{1}, \ldots, e_{k}, c\right\}$ is a ladder in $\operatorname{read}(T)$. Note that $b$ cannot be any of $e_{i}$ since any element between $b$ and $c$ in $\operatorname{read}(T)$ is bigger than $c$ with respect to $\mathcal{P}$. However, this means that $b$ is climbing the ladder $\left\{a, e_{1}, \ldots, e_{k}, c\right\}$ which contradicts the assumption.

Now let $d_{1}, d_{2}, \ldots, d_{s}$ be the elements between $a$ and $c$ in the row of $T$ containing $a$ and $c$. We also set $d_{0}=a$ and $d_{s+1}=c$ for simplicity. (Note that $d_{0} d_{1} \cdots d_{s} d_{s+1}$ is a subword of $\operatorname{read}(T)$.) Then by the condition of $\mathcal{P}$-tableaux, we have $d_{i} t_{p_{\mathcal{P}}} d_{i+1}$ for $i \in[0, s]$. (In particular, we have $s \geq 1$.) We claim that there exists $e_{1} \in\left\{d_{1}, \ldots, d_{s}\right\}$ such that $d_{0}--_{\mathcal{P}} e_{1}$. Suppose otherwise, then as $d_{0} \rightarrow_{\mathcal{P}} d_{s+1}$ and $d_{0} \succ_{\mathcal{P}} d_{1}$, there exists $j \in[1, k]$ such that $d_{0} \leftarrow_{\mathcal{P}} d_{j}$ and $d_{0} \rightarrow_{\mathcal{P}} d_{j+1}$. However, this is impossible since $d_{j} \dashv_{\mathcal{P}} d_{j+1}$.

Since $d_{0---\mathcal{P}} e_{1}$ and $d_{0} \rightarrow \mathcal{P} d_{s+1}$, we have $e_{1}>d_{s+1}$ by Condition ( $\pitchfork$ ). If $e_{1} \rightarrow_{-\rightarrow \mathcal{P}} d_{s+1}$ then it contradicts that $(a, c)=\left(d_{0}, d_{s+1}\right) \in \mathrm{g}^{-h t} \mathcal{P}(\operatorname{read}(T))$, and thus we should have $e_{1} \rightarrow_{\mathcal{P}} d_{s+1}$. Now we can iterate this process forever and find $e_{1}, e_{2}, \ldots \in\left\{d_{1}, \ldots, d_{s}\right\}$ such that $e_{1}-\mathcal{p}_{\mathcal{p}} e_{2}-\boldsymbol{p}_{\mathcal{p}} \cdots$. This is clearly a contradiction, and thus we conclude that $\operatorname{g-ht}_{\mathcal{P}}(\operatorname{read}(T))=l$ which is what we want to prove. 
9.6. Proof of Theorem 6.1] $[\mathrm{E})$, Let $\mathcal{P}-\mathrm{RS}(w)=\left(P T^{\prime}, Q T^{\prime}\right)$. We first show that $P T^{\prime}=P T$. Let $P T=\left(P T_{1}, \ldots, P T_{k}, P T_{k+1}, \ldots, P T_{p}\right)$ where the length of $P T_{i}$ equals 1 if and only if $i>k$. Then direct calculation shows that $\Phi\left(P T_{k}+P T_{k+1}+\cdots+P T_{p}, \emptyset\right)=\left(\left(P T_{k}\right),(\infty, \ldots, \infty)+P T_{k+1}+\cdots+\right.$ $\left.P T_{p}+(\infty)\right)$. Now if we assume $P T_{i}=(\ldots, a)$ and $P T_{i+1}=(b, \ldots, c)$ for $i \in[1, k-1]$, then we have $a<b$ since otherwise $a \rightarrow_{\mathcal{P}} c$ by Condition ( $\left.\pitchfork\right)$ which contradicts the assumption that $P T$ is a $\mathcal{P}$-tableau. (Here it is crucially used that the length of $P T_{i+1}$ is $\geq 2$.) In other words, if $x$ and $y$ are processed in the same step then $x, y$ should be in the same column of $P T$. Thus by Proposition 5.5. (B), we have $\Phi(w, \emptyset)=\left(P T_{1},(\infty, \cdots, \infty)+P T_{2}+(\infty, \cdots, \infty)+P T_{3}+\cdots+(\infty, \cdots, \infty)+\right.$ $\left.P T_{k+1}+\cdots+P T_{p}+(\infty)\right)$, i.e. the first column of $P T^{\prime}$ is equal to that of $P T$. Now we iterate this argument to conclude that $P T=P T^{\prime}$ as desired.

It remains to show that $Q T^{\prime}=\omega\left(T_{\lambda}\right)$. By part (B) of the theorem, if we set $l_{1}, \ldots, l_{p}$ to be the column lengths of $\lambda$ then we have $\operatorname{des}\left(Q T^{\prime}\right)=\left\{n-x \mid x \in[1, n-1], x \neq \sum_{i=1}^{k} l_{i}\right.$ for some $\left.k \in[1, p]\right\}$. By the property of evacuation, it follows that $\operatorname{des}\left(\omega\left(Q T^{\prime}\right)\right)=[1, n-1]-\left\{\sum_{i=1}^{k} l_{i} \mid k \in[1, p]\right\}$. Now the result follows from the fact that $T_{\lambda}$ is the only standard Young tableau of shape $\lambda$ that satisfies this property.

9.7. Proof of Theorem 6.1] $[\mathrm{F})$, Suppose that $\mathcal{P}-\mathrm{RS}(\alpha)=\mathcal{P}-\mathrm{RS}\left(\alpha^{\prime}\right)=(P T, Q T)$ where $P T=$ $\left(P T_{1}, \ldots, P T_{p}\right) \in \mathcal{P}-\mathrm{Tab}_{\lambda}$ and $Q T=\left(Q T_{1}, \ldots, Q T_{p}\right) \in \mathrm{SYT}_{\lambda}$. Also, let $\alpha=\alpha_{0}, \alpha_{1}, \ldots, \alpha_{p}=$ $(\infty, \ldots, \infty)$ and $\alpha^{\prime}=\alpha_{0}^{\prime}, \alpha_{1}^{\prime}, \ldots, \alpha_{p}^{\prime}=(\infty, \ldots, \infty)$ be the elements in $\mathfrak{A}$ such that $\Phi\left(\alpha_{i}, \emptyset\right)=$ $\left(P T_{i+1}, \alpha_{i+1}\right)$ and $\Phi\left(\alpha_{i}^{\prime}, \emptyset\right)=\left(P T_{i+1}, \alpha_{i+1}^{\prime}\right)$ for $i \in[0, p-1]$. Clearly $\alpha_{p}=\alpha_{p}^{\prime}$, and thus it suffices to show that $\alpha_{i+1}=\alpha_{i+1}^{\prime} \Rightarrow \alpha_{i}=\alpha_{i}^{\prime}$ for $i \in[0, p-1]$. However it follows from Lemma 8.4 since $\left(\emptyset, \widehat{\alpha_{i}}\right)=\Psi_{X_{i+1}}^{\widehat{\mathcal{P}}}\left(\widehat{\alpha_{i+1}}, P T_{i+1}\right)$ and $\left(\emptyset, \widehat{\alpha_{i}^{\prime}}\right)=\Psi_{X_{i+1}}^{\widehat{\mathcal{P}}}\left(\widehat{\alpha_{i+1}^{\prime}}, P T_{i+1}\right)$ where $X_{i+1}=\{|\alpha|+1-j \mid j \in$ $\left.Q T_{i+1}\right\}$.

9.8. Proof of Theorem 6.1 $\left[(\mathrm{G})\right.$, By part $(\mathrm{F})$ of the theorem, it suffices to show that $\mathcal{P}$-RS : $\mathfrak{S}_{n} \rightarrow$ $\bigsqcup_{\lambda \vdash n} \mathcal{P}-\mathrm{Tab}_{\lambda} \times \mathrm{SYT}_{\lambda}$ is surjective. We have (here $\omega$ is Schützenberger's evacuation)

$$
\begin{aligned}
& \sum_{\lambda \vdash n} \sum_{P T \in \mathcal{P}-\mathrm{Tab}_{\lambda}} \sum_{Q T \in \mathrm{SYT}_{\lambda}} F_{\operatorname{des}(\omega(Q T))} \sum_{\lambda \vdash n} \sum_{P T \in \mathcal{P}-\mathrm{Tab}_{\lambda}} \sum_{Q T \in \mathrm{SYT}_{\lambda}} F_{\operatorname{des}(Q T)} \quad\left(\because \omega \text { is an involution on } \mathrm{SYT}_{\lambda}\right) \\
& =\sum_{\lambda \vdash n}\left|\mathcal{P}-\mathrm{Tab}_{\lambda}\right| \cdot s_{\lambda} \\
& =\sum_{w \in \mathfrak{S}_{n}} F_{\operatorname{des} \mathcal{P}(w)} \quad(\because \text { Ges84] }) \\
& =\sum_{(P T, Q T) \in \operatorname{im} \mathcal{P}-\mathrm{RS}} F_{\operatorname{des}(\omega(Q T))}, \quad(\because \operatorname{part}[\mathrm{B}) \text { and injectivity of } \mathcal{P}-\mathrm{RS}) \\
& =\sum_{\lambda \vdash n} \sum_{P T \in \mathcal{P}-\operatorname{Tab}_{\lambda}(P T, Q T) \in \operatorname{im} \mathcal{P}-\mathrm{RS}} F_{\operatorname{des}(\omega(Q T)) .}
\end{aligned}
$$

This equality holds only when $\mathcal{P}$-RS is surjective, from which the result follows.

Remark. It is possible to prove surjectivity of $\mathcal{P}$-RS without relying on Gasharov's result. Indeed, instead one may argue similarly to the proof of Proposition [5.6 and use properties of $\mathcal{P}$-tableaux 
for $\mathcal{P}$ which avoids $\mathcal{P}_{(3,1,1), 5}$ and $\mathcal{P}_{(4,2,1,1), 6}$. Or conversely, from the theorem above we obtain the following bi-product.

Theorem 9.5. Suppose that $\mathcal{P}$ avoids $\mathcal{P}_{(3,1,1), 5}$ and $\mathcal{P}_{(4,2,1,1), 6}$. Let $P T=\left(P T_{1}, \ldots, P T_{l}\right) \in \mathcal{P}$-Tab ${ }_{\lambda}$ and $Q T=\left(Q T_{1}, \ldots, Q T_{l}\right) \in \mathrm{SYT}_{\lambda}$ for some $\lambda \vdash n$. Define $\alpha_{0}, \alpha_{1}, \ldots, \alpha_{l} \in \mathfrak{A}, c_{0}, c_{1}, \ldots, c_{l} \in \mathfrak{C}$ successively so that $\alpha_{l}=(\infty, \ldots, \infty),\left|\alpha_{l}\right|=n$, and $\Psi_{X_{i}}^{\widehat{P}}\left(\widehat{\alpha_{i}}, \widehat{P T_{i}}\right)=\left(\widehat{c_{i-1}}, \widehat{\alpha_{i-1}}\right)$ for $i \in[1, l]$ where $X_{i}=\left\{n+1-x \mid x \in \underline{Q T_{i}}\right\}$. Then we have $c_{0}=c_{1}=\cdots=c_{l}=\emptyset$, $\mathcal{P}-\operatorname{RS}\left(\alpha_{0}\right)=(P T, Q T)$, and $\alpha_{0} \in \mathfrak{S}_{n}$.

Proof. By Theorem 6.1] $(\mathrm{G})$, there exists $w \in \mathfrak{S}_{n}$ such that $\mathcal{P}-\operatorname{RS}(w)=(P T, Q T)$. It means that there exists $w=w_{0}, w_{1}, \ldots, w_{l}=(\infty, \ldots, \infty)$ such that $\Phi\left(w_{i-1}, \emptyset\right)=\left(P T_{i}, w_{i}\right)$ and $Q T_{i}=\{j \in$ $\left.[1, n] \mid\left(w_{i-1}\right)_{j} \neq \infty,\left(w_{i}\right)_{j}=\infty\right\}$ for $i \in[1, l]$ where $\left(w_{i-1}\right)_{j}$ and $\left(w_{i}\right)_{j}$ are the $j$-th coordinates of $w_{i-1}$ and $w_{i}$, respectively. Now the result follows from successively applying Lemma 8.4 .

9.9. Proof of Theorem 4.13. Suppose that $\Gamma=\left(V, \operatorname{des} \mathcal{P},\left\{E_{i}\right\}\right)$ is a connected $\mathcal{P}$-Knuth equivalence graph. We claim that $P T \in\left\{P T_{1}, \ldots, P T_{k}\right\}$ if $\mathcal{P}-\mathrm{RS}(w)=(P T, Q T)$ for some $w \in V$. Indeed, we have $\operatorname{read}(P T) \sim_{\mathcal{P}} w \sim_{\mathcal{P}} w_{i} \sim_{\mathcal{P}} \operatorname{read}\left(P T_{i}\right)$ for any $i \in[1, k]$ by Theorem6.1[C). By assumption, it means that $\operatorname{read}(P T) \in\left\{\operatorname{read}\left(P T_{1}\right), \ldots, \operatorname{read}\left(P T_{k}\right)\right\}$. Since a $\mathcal{P}$-tableau is completely determined by its reading word, it means that $P T \in\left\{P T_{1}, \ldots, P T_{k}\right\}$ as desired.

It is clear that $\mathfrak{S}_{n}$ is a disjoint union of connected $\mathcal{P}$-Knuth equivalence graphs. Since $\mathcal{P}$-RS is a bijection between $\mathfrak{S}_{n}$ and $\bigsqcup_{\lambda \vdash n} \mathcal{P}-\mathrm{Tab}_{\lambda} \times \mathrm{SYT}_{\lambda}$, it follows that $\mathcal{P}-\mathrm{RS}(V)=\bigsqcup_{i=1}^{k}\left\{P T_{i}\right\} \times \mathrm{SYT}_{\lambda_{i}}$. Thus we have (here $\left|\operatorname{f}_{\text {-inv }}(V)\right|$ is a fake $\mathcal{P}$-inversion number of any $w \in V$ and $\omega$ is Schützenberger's evacuation)

$$
\begin{array}{rlr}
\gamma_{V} & =t^{\left|\mathrm{f}-\operatorname{inv}_{\mathcal{P}}(V)\right|} \sum_{w \in V} F_{\operatorname{des} \mathcal{P}(w)} \\
& =t^{\left|\mathrm{f}-\operatorname{inv}_{\mathcal{P}}(V)\right|} \sum_{(P T, Q T) \in \mathcal{P}-\mathrm{RS}(V)} F_{\operatorname{des}(\omega(Q T))} & (\because \text { Theorem 6.1] (B) and injectivity of } \mathcal{P} \text {-RS }) \\
& =t^{\left|\mathrm{f}-\operatorname{inv}_{\mathcal{P}}(V)\right|} \sum_{i=1}^{k} \sum_{T \in \mathrm{SYT}_{\lambda_{i}}} F_{\operatorname{des}(\omega(T))} & \left(\because \mathcal{P}-\mathrm{RS}(V)=\bigsqcup_{i=1}^{k}\left\{P T_{i}\right\} \times \mathrm{SYT}_{\lambda_{i}}\right) \\
& =t^{\left|\mathrm{f}-\operatorname{inv}_{\mathcal{P}}(V)\right|} \sum_{i=1}^{k} \sum_{T \in \mathrm{SYT}_{\lambda_{i}}} F_{\operatorname{des}(T)} & \left(\because \omega \text { is an involution on each } \mathrm{SYT}_{\lambda_{i}}\right) \\
& =t^{\left|\mathrm{f}-\operatorname{inv}_{\mathcal{P}}(V)\right|} \sum_{i=1}^{k} s_{\lambda_{i}}
\end{array}
$$

as desired.

Finally, the last sentense of the theorem follows directly from Theorem 6.1](D)

\section{REFERENCES}

[AB12] Sami H. Assaf and Sara C. Billey, Affine dual equivalence and $k$-Schur functions, J. Comb. 3 (2012), no. 3, 343-399.

[Ass15] Sami H. Assaf, Dual equivalence graphs I: A new paradigm for Schur positivity, Forum Math. Sigma 3 (2015), no. 12, 1-33. 
[Ass17] _ Dual equivalence graphs II: Transformations on locally Schur positive graphs, Available at https://arxiv.org/abs/1704.07039 (2017).

[BC18] Patrick Brosnan and Timothy Y. Chow, Unit interval orders and the dot action on the cohomology of regular semisimple Hessenberg varieties, Adv. Math. 329 (2018), 955-1001. MR 3783432

[BF17] Jonah Blasiak and Sergey Fomin, Noncommutative Schur functions, switchboards, and Schur positivity, Selecta Math. (N.S.) 23 (2017), no. 1, 727-766. MR 3595905

[Bla16] Jonah Blasiak, What makes a Do graph Schur positive?, J. Algebraic Combin. 44 (2016), no. 3, 677-727. MR 3552904

[CH19a] Soojin Cho and Jaehyun Hong, Positivity of chromatic symmetric functions associated with Hessenberg functions of bounce number 3, Available at https://arxiv.org/abs/1910.07308 (2019).

[CH19b] Soojin Cho and Jisun Huh, On e-positivity and e-unimodality of chromatic quasi-symmetric functions, SIAM J. Discrete Math. 33 (2019), no. 4, 2286-2315.

[Chm15] Michael Chmutov, Type A molecules are Kazhdan-Lusztig, J. Algebraic Combin. 42 (2015), no. 4, 10591076. MR 3417258

[Cho99] Timothy Y. Chow, Descents, quasi-symmetric functions, Robinson-Schensted for posets, and the chromatic symmetric function, J. Algebraic Combin. 10 (1999), no. 3, 227-240. MR 1723185

[DvW18] Samantha Dahlberg and Stephanie van Willigenburg, Lollipop and lariat symmetric functions, SIAM J. Discrete Math. 32 (2018), no. 2, 1029-1039.

[Gas96] Vesselin Gasharov, Incomparability graphs of (3+1)-free posets are s-positive, Discrete Math. 157 (1996), 193-197.

[Ges84] Ira M. Gessel, Multipartite P-partitions and inner products of skew Schur functions, Contemp. Math. 34 (1984), 289-301.

[GP13] Mathieu Guay-Paquet, A modular relation for the chromatic symmetric functions of (3+1)-free posets, Available at https://arxiv.org/abs/1306.2400 (2013).

[GP16] A A second proof of the Shareshian-Wachs conjecture, by way of a new Hopf algebra, Available at https://arxiv.org/abs/1601.05498 (2016).

[GS01] David D. Gebhard and Bruce E. Sagan, A chromatic symmetric function in noncommuting variables, J. Algebraic Combin. 13 (2001), 227-255.

[HP19] Megumi Harada and Martha E. Precup, The cohomology of abelian Hessenberg varieties and the StanleyStembridge conjecture, Algebr. Comb. 2 (2019), no. 6, 1059-1108, Revised edition of [ MR3940624]. MR 4049838

[Rob14] Austin Roberts, Dual equivalence graphs revisited and the explicit Schur expansion of a family of LLT polynomials, J. Algebraic Combin. 39 (2014), no. 2, 389-428. MR 3159257

[SS58] Dana Scott and Patrick Suppes, Foundational aspects of theories of measurement, J. Symb. Log. 23 (1958), no. 2, 113-128.

[SS93] Richard P. Stanley and John R. Stembridge, On immanants of Jacobi-Trudi matrices and permutations with restricted position, J. Combin. Theory Ser. A 62 (1993), no. 2, 261-279. MR 1207737

[SW16] John Shareshian and Michelle L. Wachs, Chromatic quasisymmetric functions, Adv. Math. 295 (2016), $497-551$.

[SWW97] Thomas S. Sundquist, David G. Wagner, and Julian West, A Robinson-Schensted algorithm for a class of partial orders, J. Combin. Theory Ser. A 79 (1997), no. 1, 36-52. MR 1449748

[Tym08] Julianna S. Tymoczko, Permutation actions on equivariant cohomology of flag varieties, Toric topology, Contemp. Math., vol. 460, Amer. Math. Soc., Providence, RI, 2008, pp. 365-384. MR 2428368

School of Mathematics, University of Minnesota Twin Cities, Minneapolis, MN 55455, U.S.A.

E-mail address: kim00657@umn.edu

School of Mathematics, University of Minnesota Twin Cities, Minneapolis, MN 55455, U.S.A.

E-mail address: ppylyavs@umn.edu 